Supporting Information

\title{
Synthesis of $\mathrm{Q-D-Idoseptanosyl} \mathrm{Glycosides} \mathrm{Using} \mathrm{an} \mathrm{S-Phenyl} \mathrm{Septanoside} \mathrm{Donor}$
}

\author{
Steve Castro ${ }^{\dagger}$ W. Sean Fyvie, ${ }^{\dagger}$ Susan A. Hatcher, ${ }^{\S}$ and Mark W. Peczuh ${ }^{\dagger *}$ \\ ${ }^{\dagger}$ Department of Chemistry, The University of Connecticut, 55 North Eagleville Road, Storrs, CT 06269 \\ ${ }^{\S}$ Department of Chemistry, The Ohio State University, 100 West $18^{\text {th }}$ Avenue, Columbus, OH 43210 \\ Corresponding author: mark.peczuh@uconn.edu FAX: 860-486-2981
}

Total Pages: 51

\section{Contents}

Page(s) Item

$\mathrm{S} 2$

General Experimental Procedures

S2-S9

Experimental Procedures and Tabulated Data for 4, 13-24

S10-S51

NMR $\left({ }^{1} \mathrm{H}\right.$ and ${ }^{13} \mathrm{C}$ ) data for 4, 13-24, 13-Ac, 23(over-reduction) 


\section{Experimental Section}

General Unless stated otherwise, all reactions were conducted at room temperature (rt) under nitrogen atmosphere. Reactions were monitored by TLC (silica gel, $60 \AA$ A, $\mathrm{F}_{254}, 250 \mathrm{~mm}$ ). Visualization was conducted either under UV light or by charring with $2.5 \%$-anisaldehyde in $\mathrm{H}_{2} \mathrm{SO}_{4}$, acetic acid, and ethanol solution. Preparative chromatography was conducted on silica gel (60 A, 32-63 mm, Sorbent Technologies, Atlanta, GA). Melting points are uncorrected. Optical rotations were measured at $22 \pm 2$ ${ }^{\circ} \mathrm{C}$. ${ }^{1} \mathrm{H}$ NMR spectra were collected at 500, 400, and $300 \mathrm{MHz}$ with chemical shifts referenced to $\left(\mathrm{CH}_{3}\right)_{4} \mathrm{Si}\left(\square_{\mathrm{H}} 0.00 \mathrm{ppm}\right)$, the residual signal in $\mathrm{CHCl}_{3}\left(\square_{\mathrm{H}} 7.27 \mathrm{ppm}\right) .{ }^{13} \mathrm{C}$ NMR were collected at 125 and $100 \mathrm{MHz}$ and referenced to the residual signal in $\mathrm{CDCl}_{3}\left(\square_{\mathrm{c}} 77.2 \mathrm{ppm}\right)$.

Phenyl 2,3,4,5-tetra-O-benzyl-1-thio- $\square$-D-idoseptanoside (4a) Alcohol 6 (0.120 g, 0.22 mmol) was dissolved in DMF $(5 \mathrm{~mL})$ and cooled to $0^{\circ} \mathrm{C}$. To the mixture was added $\mathrm{NaH}(0.016 \mathrm{~g}, 0.66 \mathrm{mmol})$ followed by the dropwise addition of benzyl bromide $(0.06 \mathrm{~g}, 0.29 \mathrm{mmol})$. The reaction mixture was warmed to room temperature and after $3 \mathrm{~h}$ was quenched with a sat'd solution of $\mathrm{NaHCO}_{3}$. The mixture was extracted with DCM (3 x $30 \mathrm{~mL})$ and the organic layer washed with a sat'd solution of $\mathrm{NaCl}(3 \mathrm{x}$ $30 \mathrm{~mL}$ ). Afterwards the organic layer was collected and dried with $\mathrm{Na}_{2} \mathrm{SO}_{4}$ and concentrated to give a crude residue which was purifed by chromatography (15:85 EtOAc:hexane) to obtain the product (4a) as a white solid $(0.121 \mathrm{~g} 87 \%)$. m.p. $92.5-94.0^{\circ} \mathrm{C} ;[\square]_{\mathrm{D}}+64.4^{\circ}\left(\mathrm{c} 0.39, \mathrm{CHCl}_{3}\right) ;{ }^{1} \mathrm{H} \mathrm{NMR}\left(\mathrm{CDCl}_{3}\right) 400$ $\mathrm{MHz} \square 7.67(\mathrm{~d}, \mathrm{~J}=8.1 \mathrm{~Hz}, 2 \mathrm{H}), 7.49-7.43(\mathrm{~m}, 23 \mathrm{H}), 5.41(\mathrm{~d}, \mathrm{~J}=8.2 \mathrm{~Hz}, 1 \mathrm{H}), 5.09(\mathrm{~d}, \mathrm{~J}=10.6,10.6$ $\mathrm{Hz}, 1 \mathrm{H}), 5.06(\mathrm{~d}, \mathrm{~J}=10.6,10.6 \mathrm{~Hz}, 1 \mathrm{H}), 5.00(\mathrm{~d}, \mathrm{~J}=10.8 \mathrm{~Hz}, 1 \mathrm{H}), 4.95(\mathrm{~d}, \mathrm{~J}=10.8 \mathrm{~Hz}, 1 \mathrm{H}), 4.92(\mathrm{~d}, \mathrm{~J}=$ $10.8 \mathrm{~Hz}, 1 \mathrm{H}), 4.85$ (d, J = 11.2 Hz, 1H), 4.79 (d, J = 11.4 Hz, 1H), 4.13 (dd, J = 12.5, $9.5 \mathrm{~Hz}, 1 \mathrm{H}), 4.01$ $(\mathrm{dd}, \mathrm{J}=8.4,8.4 \mathrm{~Hz}, 1 \mathrm{H}), 3.94-3.82(\mathrm{~m}, 3 \mathrm{H}), 3.17(\mathrm{~s}, 1 \mathrm{H}) ;{ }^{13} \mathrm{C} \mathrm{NMR}\left(\mathrm{CDCl}_{3}\right) 100 \mathrm{MHz} \square 138.9,138.7$, 138.3(2), 135.0, 131.9, 129.9, 129.1, 128.6, 128.5, 128.4(3), 128.1, 128.0, 127.9, 127.7(2), 127.4, 91.0, 86.6, 82.9, 82.4, 80.0, 76.1, 76.0, 73.6, 61.7; EMS m/z $(\mathrm{M}+\mathrm{Na})^{+}$calcd 655.2489, found 655.2495; Anal. calcd for $\mathrm{C}_{40} \mathrm{H}_{40} \mathrm{O}_{5} \mathrm{~S}$ : C, 75.92; H, 6.37; O, 12.64; S, 5.07; found C, 76.01; H, 6.51; S, 4.89.

Phenyl 2-O-acetyl-3,4,5-tri-O-benzyl-1-thio- $\square$-D-idoseptanoside (4b) Alcohol 6 ( 0.120 g, 0.22 mmol) was dissolved in pyridine $(5 \mathrm{~mL})$ and cooled to $0^{\circ} \mathrm{C}$. To the mixture acetic anhydride $(0.042$ $\mathrm{mL}, .44 \mathrm{mmol}$ ) was added dropwise and the mixture was warmed to $\mathrm{rt}$. After $1 \mathrm{~h}$, the mixture was quenched with water and extracted with DCM $(3 \times 30 \mathrm{~mL})$. The organic layer washed with a saturated solution of $\mathrm{NaCl}(3 \times 30 \mathrm{~mL})$, dried with $\mathrm{Na}_{2} \mathrm{SO}_{4}$, and concentrated to give a crude residue which was purifed by chromatography (15:85 EtOAc:hexane) to obtain the product (4b) as a white solid (0.114 g, 
89\%). $[\square]_{\mathrm{D}}+75.4^{\circ}$ (c $\left.0.42, \mathrm{CHCl}_{3}\right)$; IR (KBr) cm $\mathrm{cm}^{-1} 3030.59,2896.56,1749.12,1228.43,1132.01$, 1072.23, 1026.91, 738.60, 697.14; ${ }^{1} \mathrm{H} \mathrm{NMR}\left(\mathrm{CDCl}_{3}\right) 400 \mathrm{MHz} \square$ 7.49-7.47 (m, 2H), 7.32-7.20 (m, $18 \mathrm{H}), 5.31(\mathrm{dd}, \mathrm{J}=8.4,8.4 \mathrm{~Hz}, 1 \mathrm{H}), 5.22(\mathrm{~d}, \mathrm{~J}=8.4 \mathrm{~Hz}, 1 \mathrm{H}), 4.82(\mathrm{~d}, \mathrm{~J}=11.4 \mathrm{~Hz}, 1 \mathrm{H}), 4.80(\mathrm{~d}, \mathrm{~J}=10.5$ $\mathrm{Hz}, 1 \mathrm{H}), 4.75-4.72(\mathrm{~m}, 2 \mathrm{H}), 4.68(\mathrm{~d}, \mathrm{~J}=11.4 \mathrm{~Hz}, 1 \mathrm{H}), 4.67(\mathrm{~d}, \mathrm{~J}=11.4 \mathrm{~Hz}, 1 \mathrm{H}), 4.02$ (dd, J = 12.0, 9.5 $\mathrm{Hz}, 1 \mathrm{H}), 3.84-3.71(\mathrm{~m}, 3 \mathrm{H}), 3.68(\mathrm{~d}, \mathrm{~J}=3.9 \mathrm{~Hz}, 1 \mathrm{H}) 1.93(\mathrm{~s}, 3 \mathrm{H}) ;{ }^{13} \mathrm{C} \mathrm{NMR}\left(\mathrm{CDCl}_{3}\right) 100 \mathrm{MHz} \square 169.5$, 138.5, 138.2(2), 134.5, 131.6, 129.4, 129.2, 129.0, 128.4(2), 128.3, 128.1, 128.0, 127.8, 127.6, 127.5, $127.4,127.1,88.8,86.4,79.9,79.7,76.6,75.9,75.4,73.5,72.3,61.4,20.9 ; \mathrm{EMS} \mathrm{m} / \mathrm{z}(\mathrm{M}+\mathrm{H})^{+}$calcd 607.2125, found 607.2118; Anal. calcd for $\mathrm{C}_{35} \mathrm{H}_{36} \mathrm{O}_{6} \mathrm{~S}: \mathrm{C}, 71.89 ; \mathrm{H}, 6.21 ; \mathrm{O}, 16.42 ; \mathrm{S}, 5.48$; found C, $72.01 ; \mathrm{H}, 6.17 ; \mathrm{S}, 5.66$.

\section{General Glycosylation Procedure}

The donor, either $\mathbf{4 a}$ or $\mathbf{4 b}(0.10 \mathrm{mmol})$, was azeotroped with the designated acceptor $(2-3 \mathrm{eq}$., see below) in toluene ( $3 \times 10 \mathrm{~mL})$. The mixture was dissolved in dry DCM (3-5 mL) under $\mathrm{N}_{2}$ and $4 \AA$ mol. sieves $(50 \mathrm{mg})$ were added. The mixture was cooled to $-40^{\circ} \mathrm{C}\left(\mathrm{CO}_{2} / \mathrm{CH}_{3} \mathrm{CN}\right)$ and solid $N$ iodosuccinimide $(0.125 \mathrm{mmol})$ and silver triflate $(0.033 \mathrm{mmol})$ were added simultaneously. Stirring was continued until the donor was consumed (as determined by TLC) and the reaction mixture turned magenta in color (aprox. $20 \mathrm{~min}$ ). For $\mathbf{4 a}$, activation occurred at $-40^{\circ} \mathrm{C}$, but reactions using $\mathbf{4 b}$ as donor were allowed to warm to $-25^{\circ} \mathrm{C}$ for activation to occur. The mixture was then stirred an additional $10-20 \mathrm{~min}$ (at either $-40^{\circ}$ or $-25^{\circ} \mathrm{C}$ ) followed by addition of TEA $(0.5 \mathrm{~mL})$. The reaction mixture was diluted with DCM $(2 \times 10 \mathrm{~mL})$ and filtered through celite. The filtrate was concentrated and purified by silica gel column chromatography using 2:8 EtOAc:Hex. as eluent to obtain the desired product. The following are the equivalents of acceptor used in each of the glycosylation reactions: 7 (3.0 eq.), 8 (3.0 eq.). 9 (3.0 eq.), 10 (2.0 eq.), 11 (2.5 eq.), 12 (1.5 eq.).

Decyl 2,3,4,5-tetra-O-benzyl- $\square$-D-idoseptanoside (13a) [ []$_{\mathrm{D}}+33.8^{\circ}$ (c 4.10, $\left.\mathrm{CHCl}_{3}\right)$; IR (KBr) $\mathrm{cm}^{-1} 3438.46,2925.48,1724.05,1396.21,1361.50,1243.86,1068.37,1027.87,906.38 ;{ }^{1} \mathrm{H}$ NMR $\left(\mathrm{CDCl}_{3}\right) 400 \mathrm{MHz} \square 7.78-7.25(\mathrm{~m}, 20 \mathrm{H}), 5.04(\mathrm{~d}, \mathrm{~J}=10.6 \mathrm{~Hz}, 1 \mathrm{H}), 4.92-4.62(\mathrm{~m}, 6 \mathrm{H}), 3.79-3.48(\mathrm{~m}$, $4 \mathrm{H}), 1.65(\mathrm{dd}, \mathrm{J}=6.6,7.2 \mathrm{~Hz}, 2 \mathrm{H}), 1.32(\mathrm{~m}, 17 \mathrm{H}), 0.94(\mathrm{t}, \mathrm{J}=6.7 \mathrm{~Hz}, 3 \mathrm{H}) ;{ }^{13} \mathrm{C} \mathrm{NMR}\left(\mathrm{CDCl}_{3}\right) 100 \mathrm{MHz}$ $\square 139.2,139.0,138.9,129.3,128.6,128.5,128.4$ (2), 128.2 (2), 128.1, 128.0, 127.9, 127.8, 127.7, 127.6, 127.4, 103.6, 87.2, 82.9, 81.1, 80.4, 76.5, 76.4, 75.8, 73.8, 68.4, 59.9, 32.1, 29.9, 29.8, 29.7, 29.6, 26.5, 22.9, 14.3; EMS m/z (M+Na) calcd 703.3969, found 703.3994. 
Decyl 2-O-acetyl-3,4,5-tri-O-benzyl- $\square$-D-idoseptanoside $(\mathbf{1 3 b})[\square]_{\mathrm{D}}+38.5^{\circ}$ (c 2.3, $\mathrm{CHCl}_{3}$ ); IR $(\mathrm{KBr}) \mathrm{cm}^{-1} 3448.10,3031.55,2925.48,2854.13,1752.98,1496.49,1454.06,1365.35,1232.29,1072.23$ ; ${ }^{1} \mathrm{H}$ NMR $\left(\mathrm{CDCl}_{3}\right) 400 \mathrm{MHz} \square 7.58-7.25(\mathrm{~m}, 15 \mathrm{H}), 5.20(\mathrm{dd}, \mathrm{J}=9.7,7.2 \mathrm{~Hz}, 1 \mathrm{H}), 4.93-4.60(\mathrm{~m}, 6 \mathrm{H})$, $4.59(\mathrm{~d}, \mathrm{~J}=7.0 \mathrm{~Hz}, 1 \mathrm{H}), 3.80-3.60(\mathrm{~m}, 5 \mathrm{H}), 3.55(\mathrm{dd}, \mathrm{J}=11.0,3.0 \mathrm{~Hz}, 1 \mathrm{H}), 3.44-3.35$ (m, 1H), 1.93(s, $3 \mathrm{H}), 1.70-1.51(\mathrm{~m}, 4 \mathrm{H}), 1.32-1.25(\mathrm{~m}, 12 \mathrm{H}) 0.93(\mathrm{t}, \mathrm{J}=6.4 \mathrm{~Hz}, 3 \mathrm{H}) ;{ }^{13} \mathrm{C} \mathrm{NMR}\left(\mathrm{CDCl}_{3}\right) 100 \mathrm{MHz} \square$ $169.6,138.9,138.7,138.5,129.2,128.6,128.5,128.2$, 127.9, 127.7, 127.5, 127.3, 127.1, 101.0 87.2, 80.2, 78.6, 76.6, 75.8, 73.8, 73.3, 68.2, 59.7, 32.1, 29.8, 29.6, 29.5, 26.2, 22.9, 21.0, 14.3; EMS m/z $(\mathrm{M}+\mathrm{H})^{+}$calcd 655.3605 , found 655.3613 .

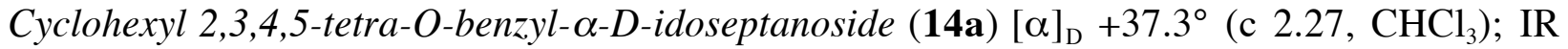
$(\mathrm{KBr}) \mathrm{cm}^{-1} 3428.81,3027.69,2927.41,2854.13,1579.41,1496.49,1452.14,1396.21,1357.64,1268.93$, 1245.79, 1214.93, 1105.01, 1064.51, 1025.94, 944.95, 910.24; ${ }^{1} \mathrm{H}$ NMR $\left(\mathrm{CDCl}_{3}\right) 400 \mathrm{MHz} \square$ 7.44-7.14 $(\mathrm{m}, 20 \mathrm{H}), 5.03(\mathrm{~d}, \mathrm{~J}=10.6 \mathrm{~Hz}, 1 \mathrm{H}), 4.90-4.61(\mathrm{~m}, 6 \mathrm{H}), 3.77-3.55(\mathrm{~m}, 6 \mathrm{H}), 1.91(\mathrm{t}, \mathrm{J}=12.8 \mathrm{~Hz}, 2 \mathrm{H}), 1.79$ $(\mathrm{d}, \mathrm{J}=9.2 \mathrm{~Hz}, 2 \mathrm{H}), 1.57-0.09(\mathrm{~m}, 8 \mathrm{H}) ;{ }^{13} \mathrm{C} \mathrm{NMR}\left(\mathrm{CDCl}_{3}\right) 100 \mathrm{MHz} \square 139.2,139.1,138.9,138.5,129.3$, 128.6, 128.5, 128.4 (2), 128.2 (2), 1128.0, 127.9, 127.7 (2), 127.6, 127.4, 101.7, 87.4, 82.9, 81.1, 80.5, 76.5, 76.2, 75.9, 75.5, 73.8, 59.7, 33.9, 32.0, 25.9, 24.3, 24.2; EMS m/z $(\mathrm{M}+\mathrm{Na})^{+}$calcd 645.3187, found 645.3173 .

Cyclohexyl 2-O-acetyl-3,4,5-tri-O-benzyl- $\square$-D-idoseptanoside $(\mathbf{1 4 b})[\square]_{\mathrm{D}}+46.6^{\circ}$ (c 2.3, $\mathrm{CHCl}_{3}$ ); IR (KBr) $\mathrm{cm}^{-1} 3448.10,3029.62,2929.34,2854.13,1737.55,1579.41,1496.49,1475.28,1452.14$, 1363.43, 1241.93, 1070.30, 950.73; ${ }^{1} \mathrm{H} \mathrm{NMR}\left(\mathrm{CDCl}_{3}\right) 400 \mathrm{MHz} \square 7.75-7.24(\mathrm{~m}, 15 \mathrm{H}), 5.19(\mathrm{dd}, \mathrm{J}=8.0$, 8.0 Hz, 1H), 4.93-4.67(m, 7H), 3.84-3.47 (m, 4H), 1.94 (s, 3H), 1.79-1.77 (m, 3H), 1.51-1.30 (m, 7H); ${ }^{13} \mathrm{C} \mathrm{NMR}\left(\mathrm{CDCl}_{3}\right) 100 \mathrm{MHz} \square 169.6,138.9,138.8,138.5,129.2,128.6,128.4,128.2,127.9,127.7$ (2), $127.5,127.3,127.2,99.2,87.2,80.3,78.6,76.6,75.8,75.3,72.8,73.4,59.6,33.5,31.5,25.8,24.0,23.7$, 21.0; EMS m/z (M+Na) calcd 597.2823, found 597.2796.

4-(t-Butyl)-phenyl 2-O-acetyl-3,4,5-tri-O-benzyl- $\square$-D-idoseptanoside $(\mathbf{1 5})[\square]_{\mathrm{D}}+41.3^{\circ}$ (c 3.7 , $\mathrm{CHCl}_{3}$ ); IR (KBr) cm $\mathrm{cm}^{-1} 3436.53,3062.41,3031.55,2960.20,2867.63,2285.23,1751.05,1606.41$, 1581.34, 1511.92, 1496.49, 1454.06, 1363.43, 1290.14, 1224.58, 1186.01, 1137.80, 1072.23, 1025.94, 906.38, 831.17; ${ }^{1} \mathrm{H} \mathrm{NMR}\left(\mathrm{CDCl}_{3}\right) 400 \mathrm{MHz} \square$ 7.59-6.99 (m,19H), 5.54 (dd, J = 8.0, 8.0 Hz, 1H), 5.34 $(\mathrm{d}, \mathrm{J}=7.1 \mathrm{~Hz}, 1 \mathrm{H}), 4.98-4.68(\mathrm{~m}, 6 \mathrm{H}), 3.93-3.71(\mathrm{~m}, 5 \mathrm{H}), 1.94(\mathrm{~s}, 3 \mathrm{H}), 1.33(\mathrm{~m}, 9 \mathrm{H}) ;{ }^{13} \mathrm{C} \mathrm{NMR}\left(\mathrm{CDCl}_{3}\right)$ $100 \mathrm{MHz} \square 169.7,154.6,145.4,138.7,138.5,138.3$, 133.7, 129.7, 129.4, 129.2, 128.6, 128.5 (2), 128.3, 
128.0, 127.9, 127.8, 127.7, 127.6, 127.3, 127.1, 126.7, 126.5, 126.1, 116.6, 99.7, 87.2, 79.9, 78.3, 76.7, 76.0, 73.8, 72.6, 60.3, 34.4, 31.7, 21.0; EMS m/z (M+Na) calcd 647.2979, found 647.2955.

\section{1,2:3,4-Di-O-isopropylidene-6-O-(2,3,4,5-tetra-O-benzyl-[-D-idoseptanosyl)-D-D-}

galactopyranose (16a) [D] $]_{\mathrm{D}}+8.15^{\circ}\left(\mathrm{c} 1.63, \mathrm{CHCl}_{3}\right)$; IR $(\mathrm{KBr}) \mathrm{cm}^{-1}$ 2987.20, 2904.27, 1727.91, 1454.06, 1373.07, 1255.43, 1211.08, 1095.37, 1068.37, 734.75, 698.11; ${ }^{1} \mathrm{H}$ NMR $\left(\mathrm{CDCl}_{3}\right) 400 \mathrm{MHz} \square$ 7.33-7.27 $(\mathrm{m}, 20 \mathrm{H}), 5.53(\mathrm{~d}, \mathrm{~J}=4.8 \mathrm{~Hz}, 1 \mathrm{H}), 5.00(\mathrm{~d}, \mathrm{~J}=10.4 \mathrm{~Hz}, 1 \mathrm{H}), 4.87(\mathrm{~d}, \mathrm{~J}=10.7 \mathrm{~Hz}, 1 \mathrm{H}), 4.85(\mathrm{~d}, \mathrm{~J}=10.4$ $\mathrm{Hz}, 1 \mathrm{H}), 4.83(\mathrm{~d}, \mathrm{~J}=10.4 \mathrm{~Hz}, 1 \mathrm{H}), 4.77-4.74(\mathrm{~m}, 3 \mathrm{H}), 4.71-4.65(\mathrm{~m}, 2 \mathrm{H}), 4.59(\mathrm{dd}, \mathrm{J}=7.8,1.4 \mathrm{~Hz}, 1 \mathrm{H})$, 4.35-4.33 (m, 1H), $4.30(\mathrm{~d}, \mathrm{~J}=7.9 \mathrm{~Hz}, 1 \mathrm{H}), 4.01(\mathrm{dd}, \mathrm{J}=6.4,6.4 \mathrm{~Hz}, 1 \mathrm{H}), 3.87-3.58(\mathrm{~m}, 7 \mathrm{H}), 1.55(\mathrm{~s}$, 3H), 1.48 (s, 3H), 1.37 (s, 3H), 1.30 (s, 3H); ${ }^{13} \mathrm{C}$ NMR $\left(\mathrm{CDCl}_{3}\right) 100 \mathrm{MHz} \mathrm{C} 139.1,138.9$ (2), 138.5, 128.6, 128.5, 128.4 (2), 128.2, 128.1, 128.0(2), 127.7, 127.6, 109.4, 108.8, 104.1, 96.6, 87.1, 82.6, 80.9, $80.2,76.5,76.4,75.7,73.8,71.0,70.9,70.7,66.6(2), 60.0,26.3,26.2,25.2,24.6 ; \quad \mathrm{EMS} \mathrm{m} / \mathrm{z}(\mathrm{M}+\mathrm{Na})^{+}$ calcd 805.3558, found 805.3559.

1,2:3,4-Di-O-isopropylidene-6-O-(2-O-acetyl,3,4,5-tri-O-benzyl-[-D-idoseptanosyl)- $\square-D$ galactopyranose $(\mathbf{1 6 b})[\square]_{\mathrm{D}}+8.47^{\circ}\left(\mathrm{c} 1.03, \mathrm{CHCl}_{3}\right) ;{ }^{1} \mathrm{H} \mathrm{NMR}\left(\mathrm{CDCl}_{3}\right) 500 \mathrm{MHz} \square$ 7.34-7.23 (m,15H), $5.52(\mathrm{~d}, \mathrm{~J}=4.9 \mathrm{~Hz}, 1 \mathrm{H}), 5.21(\mathrm{dd}, \mathrm{J}=9.1,7.5 \mathrm{~Hz}, 1 \mathrm{H}), 4.89(\mathrm{~d}, \mathrm{~J}=10.6 \mathrm{~Hz}, 1 \mathrm{H}), 4.85(\mathrm{~d}, \mathrm{~J}=11.4 \mathrm{~Hz}$, $1 \mathrm{H}), 4.80(\mathrm{~d}, \mathrm{~J}=10.6 \mathrm{~Hz}, 1 \mathrm{H}), 4.76(\mathrm{~d}, \mathrm{~J}=11.6 \mathrm{~Hz}, 1 \mathrm{H}), 4.68-4.64(\mathrm{~m}, 3 \mathrm{H}), 4.61(\mathrm{~d}, \mathrm{~J}=7.5 \mathrm{~Hz}, 1 \mathrm{H})$, $4.32(\mathrm{~m}, 1 \mathrm{H}), 4.21(\mathrm{~d}, \mathrm{~J}=8.0 \mathrm{~Hz}, 1 \mathrm{H}), 3.95(\mathrm{dd}, \mathrm{J}=6.7,6.7 \mathrm{~Hz}, 1 \mathrm{H}), 3.79-3.58(\mathrm{~m}, 7 \mathrm{H}), 1.93(\mathrm{~s}, 3 \mathrm{H})$, 1.55 (s, 3H), 1.45 (s, 3H), 1.35 (s, 3H), 1.34 (s, 3H); ${ }^{13} \mathrm{C} \mathrm{NMR}\left(\mathrm{CDCl}_{3}\right) 125 \mathrm{MHz} \square 169.4,138.7,138.5$, 138.3, 128.4, 128.5, 128.3 (2), 128.0, 127.8, 127.5, 127.4, 127.0, 109.1, 108.6, 101.6, 96.3, 86.9, 79.9, 78.3, 77.3, 76.4, 75.7, 73.4, 72.9, 70.7 (2), 70.6, 66.5, 66.0, 59.8, 26.1, 26.0, 25.0, 24.6, 20.8; EMS m/z $(\mathrm{M}+\mathrm{Na})^{+}$calcd 757.3194, found 757.3161 .

Methyl-O-(2,3,4,5-tetra-O-benzyl-[-D-idoseptanosyl)-( $1 \rightarrow 4)-2,3,6$-tri-O-benzyl-D-D-

glucopyranoside (18a) $[\square]_{\mathrm{D}}+17.6^{\circ}\left(\mathrm{c} 0.82, \mathrm{CHCl}_{3}\right)$; IR $(\mathrm{KBr}) \mathrm{cm}^{-1}$ 3029.62, 2902.34, 1637.27, 1617.98, 1097.30, 1047.16, 736.67, 698.11; ${ }^{1} \mathrm{H}$ NMR $\left(\mathrm{CDCl}_{3}\right) 400 \mathrm{MHz} \longrightarrow 7.36-7.20(\mathrm{~m}, 35 \mathrm{H}), 5.29$ (d, J = 7.2 $\mathrm{Hz}, 1 \mathrm{H}), 4.95(\mathrm{~d}, \mathrm{~J}=11.1 \mathrm{~Hz}, 1 \mathrm{H}), 4.92(\mathrm{~d}, \mathrm{~J}=11.4 \mathrm{~Hz}, 1 \mathrm{H}), 4.86(\mathrm{~d}, \mathrm{~J}=8.7 \mathrm{~Hz}, 1 \mathrm{H}), 4.83(\mathrm{~d}, \mathrm{~J}=8.2$ $\mathrm{Hz}, 1 \mathrm{H}), 4.80-4.62(\mathrm{~m}, 7 \mathrm{H}), 4.59-4.58(\mathrm{~m}, 2 \mathrm{H}), 4.45(\mathrm{~d}, \mathrm{~J}=11.4 \mathrm{~Hz}, 1 \mathrm{H}), 3.99(\mathrm{dd}, \mathrm{J}=9.1,9.1 \mathrm{~Hz}, 1 \mathrm{H})$, $3.86(\mathrm{dd}, \mathrm{J}=9.5,8.9 \mathrm{~Hz}, 1 \mathrm{H}), 3.77-3.70(\mathrm{~m}, 2 \mathrm{H}), 3.64(\mathrm{dd}, \mathrm{J}=10.6,5.1,1 \mathrm{H}), 3.58-3.53(\mathrm{~m}, 3 \mathrm{H}), 3.49-$ $3.46(\mathrm{~m}, 4 \mathrm{H}), 3.47$ (s, 3H), 3.44-3.43 (m, 1H); ${ }^{13} \mathrm{C} \mathrm{NMR}\left(\mathrm{CDCl}_{3}\right) 100 \mathrm{MHz} \square 138.9,138.7,138.4,138.3$, 138.2, 138.1, 128.5(2), 128.4, 128.3(2), 128.1, 128.0(2), 127.9, 127.8(2), 127.7, 127.5, 127.4(2), 127.3, 
127.2, 101.8, 98.0, 86.2, 82.3, 81.5, 81.1, 80.2, 76.6, 76.1, 75.9, 75.0, 73.4, 73.2, 71.5, 69.6, 69.3(2), 60.7, 55.3; EMS m/z (M+Na) calcd 1009.4497, found 1009.4503 .

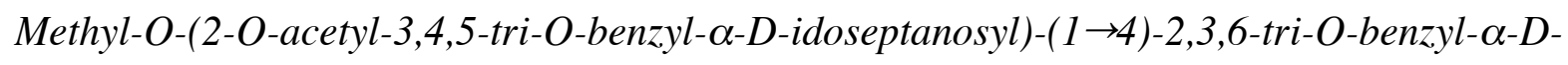
glucopyranoside (18b) [0] $]_{\mathrm{D}}+29.8^{\circ}\left(\mathrm{c} 1.25, \mathrm{CHCl}_{3}\right)$; IR (KBr) $\mathrm{cm}^{-1}$ 3030.59, 2903.31, 1750.08, 1456.96, 1364.39, 1231.33, 1045.23, 736.67, 698.11; ${ }^{1} \mathrm{H}$ NMR $\left(\mathrm{CDCl}_{3}\right) 500 \mathrm{MHz} \longrightarrow$ 7.37-7.20 (m,35H), 5.42 (d, J $=7.8 \mathrm{~Hz}, 1 \mathrm{H}), 5.15(\mathrm{dd}, \mathrm{J}=8.7,8.7 \mathrm{~Hz}, 1 \mathrm{H}), 5.07(\mathrm{~d}, \mathrm{~J}=10.6 \mathrm{~Hz}, 1 \mathrm{H}), 4.87(\mathrm{~d}, \mathrm{~J}=10.5 \mathrm{~Hz}, 1 \mathrm{H}), 4.78-$ $4.73(\mathrm{~m}, 4 \mathrm{H}), 4.65-4.57(\mathrm{~m}, 6 \mathrm{H}), 4.48(\mathrm{~d}, \mathrm{~J}=11.7 \mathrm{~Hz}, 2 \mathrm{H}), 3.94-3.87(\mathrm{~m}, 2 \mathrm{H}), 3.76-3.74(\mathrm{~m}, 1 \mathrm{H}), 3.67$ (m, 3H), $3.57(\mathrm{dd}, \mathrm{J}=8.9,3.3 \mathrm{~Hz}, 1 \mathrm{H}), 3.55-3.48(\mathrm{~m}, 3 \mathrm{H}), 3.43-3.37(\mathrm{~m}, 1 \mathrm{H}), 3.41(\mathrm{~s}, 3 \mathrm{H}), 1.86(\mathrm{~s}, 3 \mathrm{H})$; ${ }^{13} \mathrm{C} \mathrm{NMR}\left(\mathrm{CDCl}_{3}\right) 125 \mathrm{MHz} \mathrm{169.5}, 138.7,138.6,138.4,138.3,138.2,138.0,128.5,128.4(2), 128.3(3)$, 128.1, 128.0, 127.7, 127.6(3), 127.5, 127.4, 127.3, 126.9, 99.8, 97.7, 86.2, 82.5, 80.3, 80.0, 78.6, 76.3, 75.7, 75.1, 73.4, 73.3, 73.2, 72.2, 71.6, 69.2, 69.0, 60.2, 55.2, 20.9; EMS m/z (M+Na) ${ }^{+}$calcd 961.4133, found 961.4091 .

1,6-Anhydro-3,4-di-O-benzyl-5,7-O-benzylidene-2-deoxy-D-glycero-D-gulosept-1-enitol (19) m.p. 70-72 ${ }^{\circ} \mathrm{C}$; IR (KBr) cm ${ }^{-1}$ : 3087.48, 3065.30, 3028.66, 2913.91, 2868.59, 1654.62, 1390.42, 1370.18, 1279.54, 1244.83, 1213.97, 1093.44, 1027.87, 986.41, 771.39, 733.78, 692.32; ${ }^{1} \mathrm{H}$ NMR $\left(\mathrm{CDCl}_{3}\right)$ : $400 \mathrm{Mhz} \square 7.62-7.38(\mathrm{~m}, 15 \mathrm{H}), 6.44(\mathrm{~d}, \mathrm{~J}=7.2 \mathrm{~Hz}, 1 \mathrm{H}), 5.60(\mathrm{~s}, 1 \mathrm{H}), 4.95(\mathrm{dd}, 6.5,6.5 \mathrm{~Hz}$, 1H), $4.85(\mathrm{~d}, \mathrm{~J}=11.5 \mathrm{~Hz}, 1 \mathrm{H}), 4.80(\mathrm{~d}, \mathrm{~J}=9.2 \mathrm{~Hz}, 1 \mathrm{H}), 4.78(\mathrm{~d}, \mathrm{~J}=9.6 \mathrm{~Hz} 1 \mathrm{H}), 4.71(\mathrm{~d}, \mathrm{~J}=11.8 \mathrm{~Hz}$, $1 \mathrm{H}), 4.61(\mathrm{ddd}, \mathrm{J}=5.6,9.6,9.6 \mathrm{~Hz}, 1 \mathrm{H}), 4.42(\mathrm{dd}, \mathrm{J}=5.5,10.8 \mathrm{~Hz}, 1 \mathrm{H}) 4.18-4.11(\mathrm{~m} \mathrm{2H}), 4.02(\mathrm{dd}, \mathrm{J}=$ 5.2, $5.2 \mathrm{~Hz}, 1 \mathrm{H}), 3.79(\mathrm{dd}, \mathrm{J}=10.5,10.5 \mathrm{~Hz}, 1 \mathrm{H}) ;{ }^{13} \mathrm{C} \mathrm{NMR}\left(\mathrm{CDCl}_{3}\right): 100 \mathrm{Mhz} \square$ 147.2, 138.4 (2), 137.8, 129.1, 128.5 (2), 128.3, 128.2, 127.8 (3), 126.34, 105.4, 100.8, 84.0, 83.6, 74.4, 73.8, 71.4, 70.0, 68.7; FAB-MS [M-H] $]^{+} m / z$ calcd for $\mathrm{C}_{28} \mathrm{H}_{28} \mathrm{O}_{5} 443.1858$, found 443.1846; Anal. calcd for $\mathrm{C}_{28} \mathrm{H}_{28} \mathrm{O}_{5}: \mathrm{C}$, 73.95; H, 6.90; O, 19.15; found C, 75.29; H, 6.72 .

Methyl 3,4-di-O-benzyl-5,7-O-benzylidene--D-D-glycero-D-guloseptanside (20) Oxepine 19 $(.0780 \mathrm{~g}, 0.195 \mathrm{mmol})$ was dried via azeotropic distillation from toluene $(3 \times 5 \mathrm{~mL})$ under reduced pressure and dissolved in dry $\mathrm{CH}_{2} \mathrm{Cl}_{2}(5 \mathrm{~mL})$. The solution was cooled in an ice bath to $0{ }^{\circ} \mathrm{C}$ and DMDO (1.34 mL, $0.32 \mathrm{M}$ in DCM) was added dropwise. The mixture was stirred at $0{ }^{\circ} \mathrm{C}$ for $1 \mathrm{~h}$ and the solvent was removed under reduced pressure to give an off-white solid. To this solid was added a solution of $\mathrm{NaOMe}$ in $\mathrm{MeOH}(2 \mathrm{mg} / \mathrm{mL})$ and the mixture was stirred for $4-5 \mathrm{~d}$ at rt. Solvent was removed under reduced pressure and the resulting residue was purified by column chromatography, 
using 3:1 hexanes-EtOAc as eluent to give $20(0.059 \mathrm{~g}, 68 \%)$ as a yellow oil. [ $\left.\square]_{\mathrm{D}}-1.6^{\circ}(c) 1.5, \mathrm{CHCl}_{3}\right)$; IR (KBr) $\mathrm{cm}^{-1}$ : 3470.28, 3063.37, 3031.55, 2862.81, 1496.49, 1454.06, 1385.60, 1282.43, 1209.15, 1089.58, 1027.87, 736.67, 697.14; ${ }^{1} \mathrm{H} \mathrm{NMR}\left(\mathrm{CDCl}_{3}\right)$ : $400 \mathrm{Mhz} \square 7.52 \square 7.30(\mathrm{~m} \mathrm{15H}), 5.49$ (s, $\left.1 \mathrm{H}\right)$, 4.72-4.69 (m, 4H), $4.65(\mathrm{~d}, \mathrm{~J}=11.8 \mathrm{~Hz}, 1 \mathrm{H}), 4.34$ (dd, J = 5.5, $10.8 \mathrm{~Hz}, 1 \mathrm{H}), 4.16-4.08(\mathrm{~m}, 1 \mathrm{H}), 4.04$ (d, $\mathrm{J}=3.6 \mathrm{~Hz}, 1 \mathrm{H}), 3.96-3.90(\mathrm{~m}, 2 \mathrm{H}), 3.86(\mathrm{~d}, \mathrm{~J}=6.8 \mathrm{~Hz}, 1 \mathrm{H}), 3.66(\mathrm{dd}, \mathrm{J}=10.4,10.4 \mathrm{H}), 3.55(\mathrm{~s}, 3 \mathrm{H})$; ${ }^{13} \mathrm{C} \mathrm{NMR}\left(\mathrm{CDCl}_{3}\right): 100 \mathrm{Mhz} \square 138.2,137.8,129.0128 .8,128.5,128.4,128.3,128.0,127.9,127.8$, $126.3,103.6,100.8,85.8,80.5,80.4,73.9,73.4,73.1,69.6,64.1,56.8 ;$ FAB-MS $[\mathrm{M}+\mathrm{H}]^{+} \mathrm{m} / z$ calcd for $\mathrm{C}_{29} \mathrm{H}_{32} \mathrm{O}_{7}$ 493.2226, found 493.2204.

Methyl 2,3,4-tri-O-benzyl-5,7-O-benzylidene- $-D$-glycero-D-guloseptanside (21) Alcohol 20 $(0.138 \mathrm{~g} / 0.280 \mathrm{mmol})$ was dried via azeotropic distillation from toluene $(3 \mathrm{x} 5 \mathrm{~mL})$ under reduced pressure and dissolved in DMF (3 mL). $\mathrm{NaH}(60 \%$ in an oil dispersion) $(0.034 \mathrm{~g}, 0.840 \mathrm{mmol})$ was added to the solution at $0{ }^{\circ} \mathrm{C}$ under $\mathrm{N}_{2}$ with stirring. $\mathrm{BnBr}(0.263 \mathrm{~mL}, 1.68 \mathrm{mmol})$ was then added dropwise. The reaction mixture was then allowed to warm to room temperature and stirred overnight. The reaction mixture was quenched with the addition of EtOAc: $\mathrm{H}_{2} \mathrm{O}(1: 1)(15 \mathrm{~mL})$ and extracted with $\mathrm{CH}_{2} \mathrm{Cl}_{2}(3 \times 50 \mathrm{~mL})$. The combined organic layers were dried $\left(\mathrm{Na}_{2} \mathrm{SO}_{4}\right)$ and the solvent was removed under reduced pressure. Purification of the residue by column chromatography using 17:3 hexanesEtOAc as eluent gave the desired product $(0.155 \mathrm{~g}, 95 \%)$ as a yellow oil. [ []$_{\mathrm{D}}-22.4^{\circ}\left(c 1.1, \mathrm{CHCl}_{3}\right)$; IR $(\mathrm{KBr}) \mathrm{cm}^{-1}$ : 3063.37, 3031.55, 2859.92, 1497.45, 1455.03, 1365.35, 1282.43, 1208.18, 1091.51, 1026.91, 735.71, 697.14; ${ }^{1} \mathrm{H}$ NMR $\left(\mathrm{CDCl}_{3}\right)$ : $400 \mathrm{Mhz} \square$ 7.54-7.26 (m, 20H), 5.52 (s, 1H), 4.78-4.67 (m, 7H), $4.31(\mathrm{dd}, \mathrm{J}=5.5,10.7 \mathrm{~Hz}, 1 \mathrm{H}), 4.16-4.06$, (m, 2H), $3.94(\mathrm{~d}, \mathrm{~J}=7.5 \mathrm{~Hz}, 1 \mathrm{H}), 3.87-3.84(\mathrm{~m}, 2 \mathrm{H})$, $3.63(\mathrm{dd}, \mathrm{J}=10.4,10.4 \mathrm{~Hz}, 1 \mathrm{H}), 3.48(\mathrm{~s}, 3 \mathrm{H}) ;{ }^{13} \mathrm{C} \mathrm{NMR}\left(\mathrm{CDCl}_{3}\right): 100 \mathrm{Mhz} \square 138.8,138.6,138.5,137.9$, 129.0, 128.6, 128.5 (2), 128.4 (2), 128.3, 128.2, 128.0 (2), 127.8, 127.7 (2), 126.3, 103.8, 100.9, 84.9, 81.5, 80.9, 80.1, 74.8, 73.8 (2), 69.8, 63.5, 56.7; FAB-MS $[\mathrm{M}-\mathrm{H}]^{+} m / z$ calcd for $\mathrm{C}_{36} \mathrm{H}_{38} \mathrm{O}_{7}$ 581.2539, found 581.2527 .

Methyl 2,3,4,7-tetra-O-benzyl-[-D-glycero-D-guloseptanside (22) Protected septanoside 21 $(0.056 \mathrm{~g}, 0.096 \mathrm{mmol})$ was dried via azeotropic distillation from toluene $(3 \mathrm{x} 5 \mathrm{~mL})$ under reduced pressure and dissolved in $3 \mathrm{~mL}$ of solution of $\mathrm{BH}_{3} \bullet \mathrm{Me}_{2} \mathrm{NH}$ in dry $\mathrm{CH}_{3} \mathrm{CN}$. The solution was cooled to 0 ${ }^{\circ} \mathrm{C}$ and $\mathrm{BF}_{3} \cdot \mathrm{EtO}_{2}$ was added with stirring under $\mathrm{N}_{2}$ for 1.5 hours. The reaction mixture was quenched with a saturated solution of $\mathrm{NaHCO}_{3}(1 \mathrm{~mL})$. The reaction mixture was extracted with EtOAc $(3 \times 25$ 
$\mathrm{mL})$. The combined organic layers were washed with a saturated solution of $\mathrm{NaHCO}_{3}(1 \times 10 \mathrm{~mL})$ and brine $(1 \times 10 \mathrm{~mL})$. The organic layer was dried $\left(\mathrm{Na}_{2} \mathrm{SO}_{4}\right)$ and the solvent was removed under reduced pressure. Purification of the residue by column chromatography using 3:1 hexanes-EtOAc as eluent gave the desired product $(0.034 \mathrm{~g}, 61 \%)$ as a yellow oil. [ $[\square]_{\mathrm{D}}+5.3^{\circ}\left(c 5.4, \mathrm{CHCl}_{3}\right) ; \mathrm{IR}(\mathrm{KBr}) \mathrm{cm}^{-1}$ : 3546.45, 3087.48, 3062.41, 3029.62, 2921.63, 2865.70, 1750.08, 1496.49, 1454.06, 1364.39, 1207.22, 1074.16, 910.24, 735.71, 697.14; ${ }^{1} \mathrm{H}$ NMR $\left(\mathrm{CDCl}_{3}\right): 300 \mathrm{Mhz} \square 7.39-7.18$ (m, 20H), $4.84(\mathrm{dd}, \mathrm{J}=3.4$, 12.1 Hz, 2H), 4.73-4,51 (m, 7H), $3.96(\mathrm{dd}, \mathrm{J}=1.9 \mathrm{~Hz}, 7.7 \mathrm{~Hz}, 1 \mathrm{H}), 3.88-3.74(\mathrm{~m}, 4 \mathrm{H}), 3.69-3.60$ (m, $2 \mathrm{H}), \quad 3.56(\mathrm{~s}, \quad 3 \mathrm{H}), 2.60(\mathrm{~d}, \quad \mathrm{~J}=7.2 \mathrm{~Hz}, \quad 1 \mathrm{H}) ; \quad{ }^{13} \mathrm{C} \quad \mathrm{NMR} \quad\left(\mathrm{CDCl}_{3}\right): 100 \mathrm{Mhz}$ $\square 138.9,138.4$ (2), 138.3, 128.7, 128.6 (2), 128.5, 128.2, 128.1, 128.0 (3), 127.8, 107.4, 80.7, 80.3, 80.2, $78.5,74.4,74.0,73.7,73.6,73.3,72.6,56.5$;

FAB-MS $[\mathrm{M}-\mathrm{H}]^{+} m / z$ calcd for $\mathrm{C}_{36} \mathrm{H}_{40} \mathrm{O}_{7}$ 583.2696, found 583.2682.

Methyl 2,3,4,5-tetra-O-benzyl- $\square-D$-glycero-D-guloseptanside (23) The protected septanoside 21 $(0.121 \mathrm{~g}, 0.203 \mathrm{mmol})$ was dried via azeotropic distillation from toluene $(3 \mathrm{x} 5 \mathrm{~mL})$ under reduced pressure and dissolved in $4 \mathrm{~mL}$ of dry $\mathrm{CH}_{2} \mathrm{Cl}_{2}: \mathrm{Et}_{2} \mathrm{O}(1: 1)$. The solution was cooled to $0{ }^{\circ} \mathrm{C}$ and $\mathrm{LAH}$ was added with stirring under $\mathrm{N}_{2}$. The reaction was allowed to warm to room temperature and a suspension of $\mathrm{AlCl}_{3}$ in dry ether $(3 \mathrm{~mL})$ was added. The reaction was then heated under reflux conditions. After 2 hours the reaction was cooled to room temperature and quenched with EtOAc (10 $\mathrm{mL})$ and $\mathrm{H}_{2} \mathrm{O}(15 \mathrm{~mL})$. The reaction mixture was extracted with ether $(1 \times 50 \mathrm{~mL})$. The organic layer was washed with $\mathrm{H}_{2} \mathrm{O}(3 \times 20 \mathrm{~mL})$ and dried $\left(\mathrm{Na}_{2} \mathrm{SO}_{4}\right)$ and the solvent was removed under reduced pressure. Purification of the residue by column chromatography using 3:1 hexanes-EtOAc as eluent gave three fractions. The first fraction gave unreacted starting material $19(0.013 \mathrm{~g}, 11 \%)$. The second gave $22(0.016 \mathrm{~g}, 13 \%)$. The third fraction gave the desired product $23(0.050 \mathrm{~g}, 42 \%)$ as a yellow oil. $\left.[\square]_{\mathrm{D}}+15.4^{\circ}(c) 2.1, \mathrm{CHCl}_{3}\right) ; \mathrm{IR}(\mathrm{KBr}) \mathrm{cm}^{-1}: 3480.88,3087.48,3062.41,3029.62,2927.41,1719.23$, 1496.49, 1454.06, 1386.57, 1207.22, 1073.19, 1027.87, 736.67, 698.11; ${ }^{1} \mathrm{H} \mathrm{NMR}\left(\mathrm{CDCl}_{3}\right): 400 \mathrm{Mhz}$ $\checkmark$ 7.34-7.11 (m, 20H), $4.79(\mathrm{~d}, \mathrm{~J}=12.2 \mathrm{~Hz}, 1 \mathrm{H}), 4.69-4.62(\mathrm{~m}, 4 \mathrm{H}), 4.52(\mathrm{~d}, \mathrm{~J}=11.2 \mathrm{~Hz}, 1 \mathrm{H}), 4.43-4.33$ $(\mathrm{m}, 3 \mathrm{H}), 4.00(\mathrm{ddd}, \mathrm{J}=3.8,5.2,9.4 \mathrm{~Hz}, 1 \mathrm{H}), 3.88(\mathrm{~s}, 2 \mathrm{H}), 3.84(\mathrm{dd}, \mathrm{J}=3.3,11.6 \mathrm{~Hz}, 1 \mathrm{H}), 3.75(\mathrm{~d}, \mathrm{~J}=$ $6.3 \mathrm{~Hz}, \quad 1 \mathrm{H}), \quad 3.66-3.61(\mathrm{~m}, \quad 2 \mathrm{H}), \quad 3.53, \quad(\mathrm{~s}, \quad 3 \mathrm{H}) ; \quad{ }^{13} \mathrm{C} \quad \mathrm{NMR} \quad\left(\mathrm{CDCl}_{3}\right) \quad 100 \quad \mathrm{MHz}$ $\square 138.8,138.5,138.2,138.1,128.6$ (2), 128.5, 128.4, 128.3, 128.1 (2), 128.0, 127.9, 127.8, 127.7,106.6, 81.3, 79.8, 78.7, 78.5, 75.9, 73.8, 73.5, 73.1, 72.7, 64.2, 56.7;

FAB-MS [M-H] $]^{+} m / z$ calcd for $\mathrm{C}_{36} \mathrm{H}_{40} \mathrm{O}_{7}$ 583.2695, found 583.2695. 
Methyl-O-(2,3,4,5-tetra-O-benzyl-[-D-idoseptanosyl)-(1 $1 \rightarrow 7)-2,3,4,5$-tetra-O-benzyl-[-D-

glycero-D-guloseptanoside (24) Donor 4b (0.035 g, $0.06 \mathrm{mmol})$ and $23(0.023 \mathrm{~g}, 0.04 \mathrm{mmol})$ were azeotroped with toluene $(3 \times 10 \mathrm{~mL})$. The mixture was dissolved in dry DCM ( $2 \mathrm{~mL})$ under $\mathrm{N}_{2}, 4 \AA$ mol. sieves $\left(50 \mathrm{mg}\right.$ ) were added, and stirred at $\mathrm{rt}$ for $10-15 \mathrm{~min}$. The mixture was then cooled to $-40^{\circ} \mathrm{C}$ $\left(\mathrm{CO}_{2} / \mathrm{CH}_{3} \mathrm{CN}\right)$ and solid $N$-iodosuccinimide $(0.017 \mathrm{~g}, 0.074 \mathrm{mmol})$ and silver triflate $(0.005 \mathrm{~g}, 0.02$ mmol) were added simultaneously. Stirring was continued as the mixture warmed to $-25^{\circ} \mathrm{C}$ and the donor was consumed (as determined by TLC) and the reaction mixture turned magenta in color (aprox. $20 \mathrm{~min})$. The mixture was then stirred an additional $10-20 \mathrm{~min}$ at $-25^{\circ} \mathrm{C}$, and then TEA $(0.25 \mathrm{~mL})$ was added dropwise. The reaction mixture was diluted with DCM $(2 \times 5 \mathrm{~mL})$ and filtered through celite. The filtrate was concentrated and purified by silica gel column chromatography using 1:4 EtOAc:Hex. as eluent to obtain $24(0.031,76 \%)$ as a clear colorless oil. [ [ $]_{\mathrm{D}}+37.2^{\circ}\left(\mathrm{c} 1.01, \mathrm{CHCl}_{3}\right)$; IR $(\mathrm{KBr}) \mathrm{cm}^{-1}$ 3029.62, 2925.48, 1747.19, 1216.86, 1072.23, 909.27, 55.96, 699.07, 667.25; ${ }^{1} \mathrm{H}$ NMR $\left(\mathrm{CDCl}_{3}\right) 400$ MHz $\square$ 7.36-7.12 (m,35H), $5.21(\mathrm{dd}, \mathrm{J}=9.6,7.2 \mathrm{~Hz}, 1 \mathrm{H}), 4.89(\mathrm{~d}, \mathrm{~J}=10.4 \mathrm{~Hz}, 1 \mathrm{H}), 4.85(\mathrm{~d}, \mathrm{~J}=11.6$ $\mathrm{Hz}, 2 \mathrm{H}), 4.83-4.6(\mathrm{~m}, 9 \mathrm{H}), 4.49(\mathrm{~d}, \mathrm{~J}=11.1 \mathrm{~Hz}, 1 \mathrm{H}), 4.42-4.32(\mathrm{~m}, 3 \mathrm{H}), 4.03(\mathrm{ddd}, \mathrm{J}=8.9,6.7,1.4 \mathrm{~Hz}$, $1 \mathrm{H}), 3.90-3.84(\mathrm{~m}, 2 \mathrm{H}), 3.78-3.61(\mathrm{~m}, 7 \mathrm{H}), 3.56(\mathrm{~s}, 3 \mathrm{H}), 3.45(\mathrm{dd}, \mathrm{J}=9.9,2.2,1 \mathrm{H}), 1.86(\mathrm{~s}, 3 \mathrm{H}) ;{ }^{13} \mathrm{C}$ NMR ( $\left.\mathrm{CDCl}_{3}\right) 125 \mathrm{MHz} \mathrm{1}$ 169.5, 138.8, 138.7, 138.5(2), 138.2, 138.0, 136.6, 129.5, 128.5, 128.4(2), 128.3, 128.2, 128.1, 128.0, 127.8(2), 127.7, 127.5(2), 127.3, 126.9, 106.4, 101.2, 87.0, 81.4, 79.9, 79.8, $78.5,78.3,76.5,75.7,75.4,73.7,73.5,73.3,72.9,72.5,68.9,59.5,56.4,20.9 ; \mathrm{EMS} \mathrm{m}_{\mathrm{z}}(\mathrm{M}+\mathrm{Na})^{+} \mathrm{calcd}$ 1081.4709, found 1081.4734. 

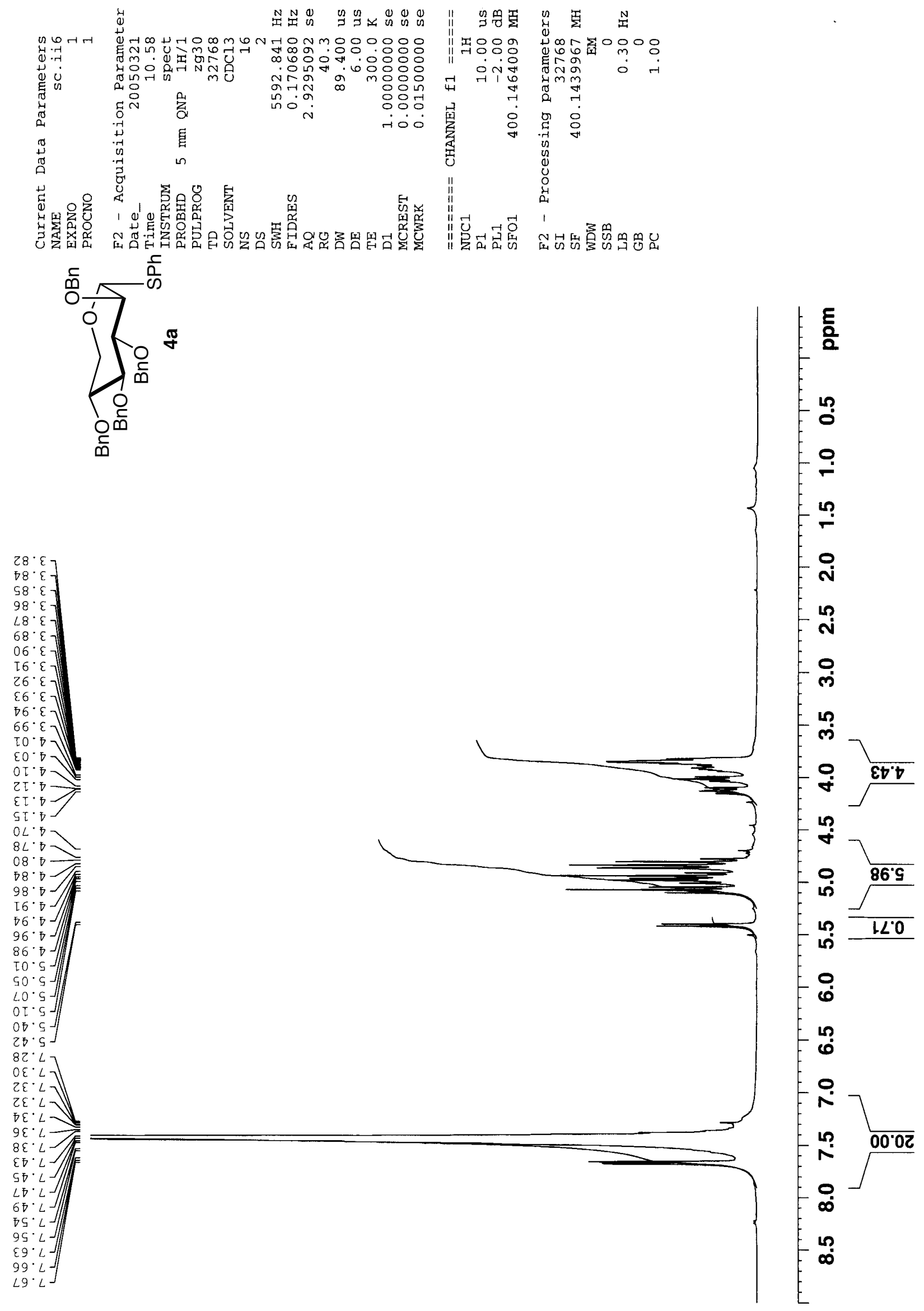

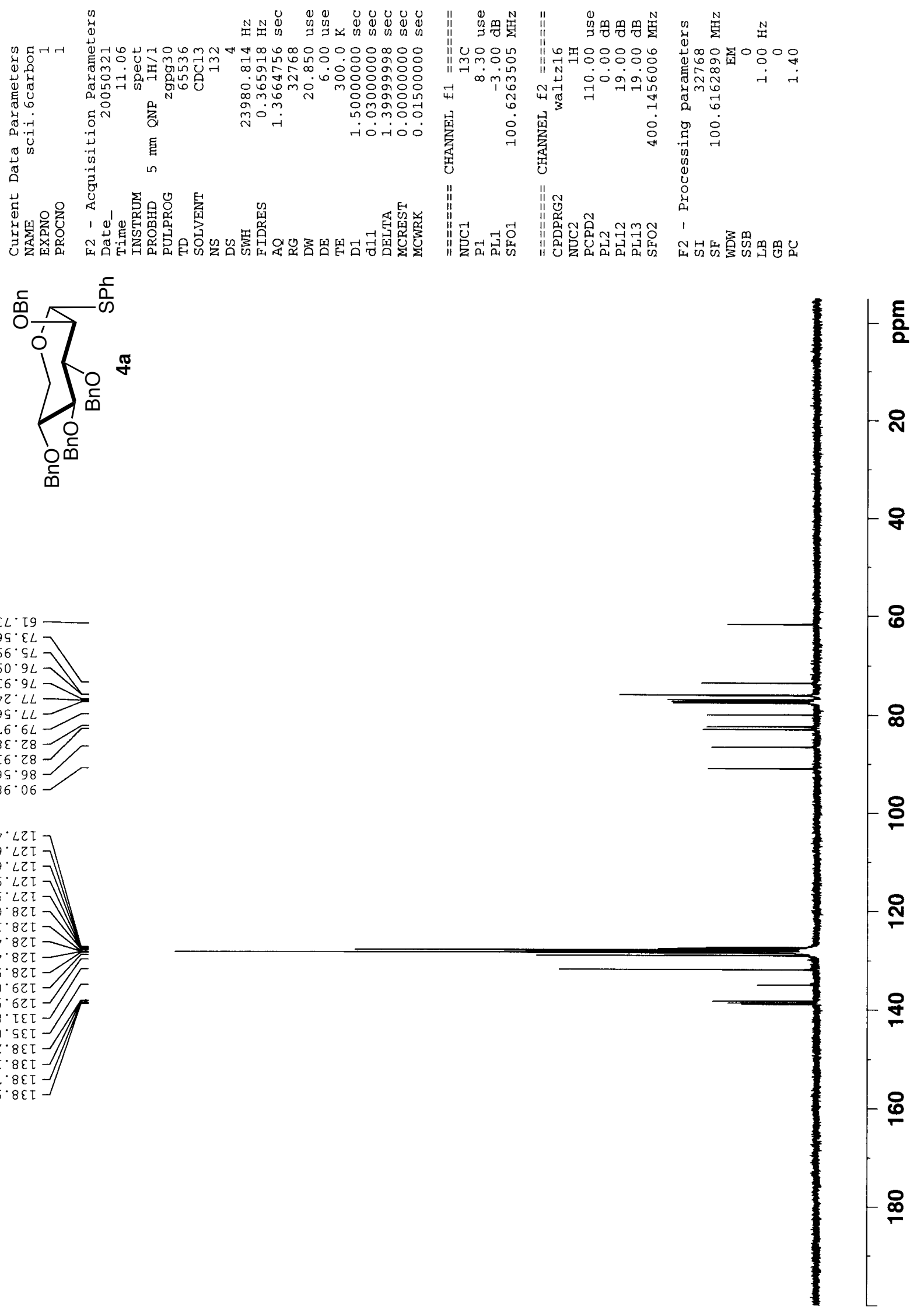


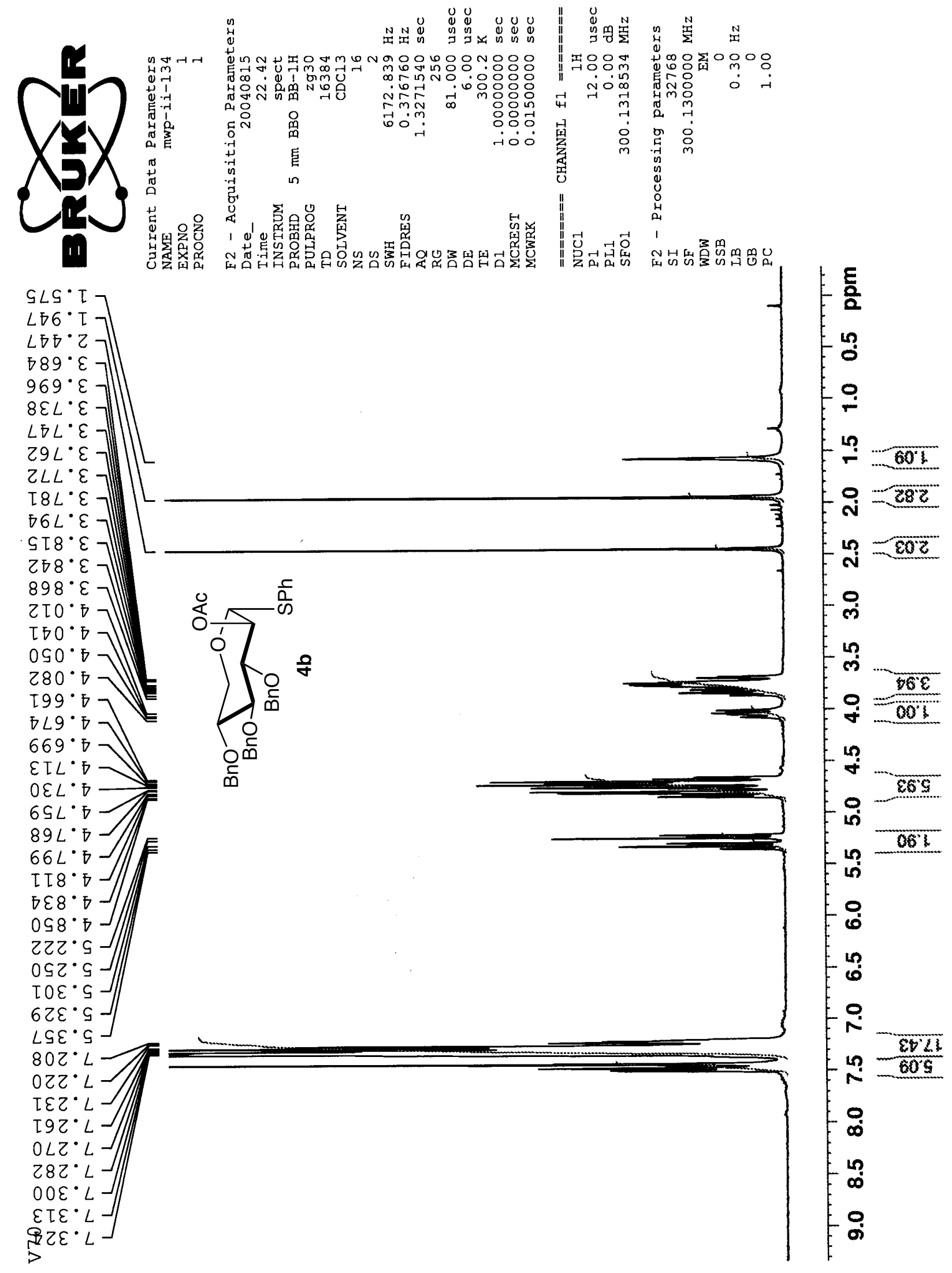




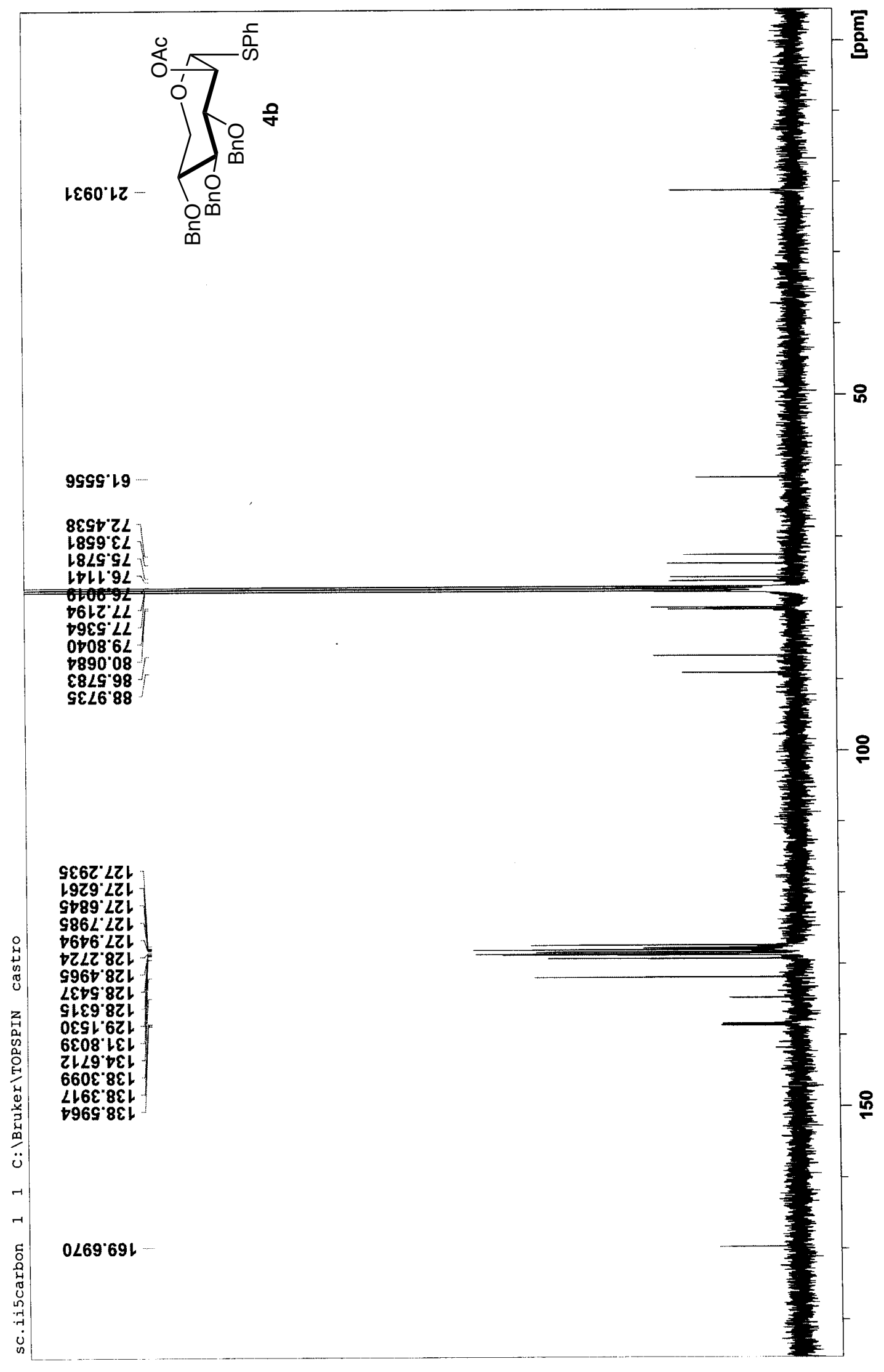



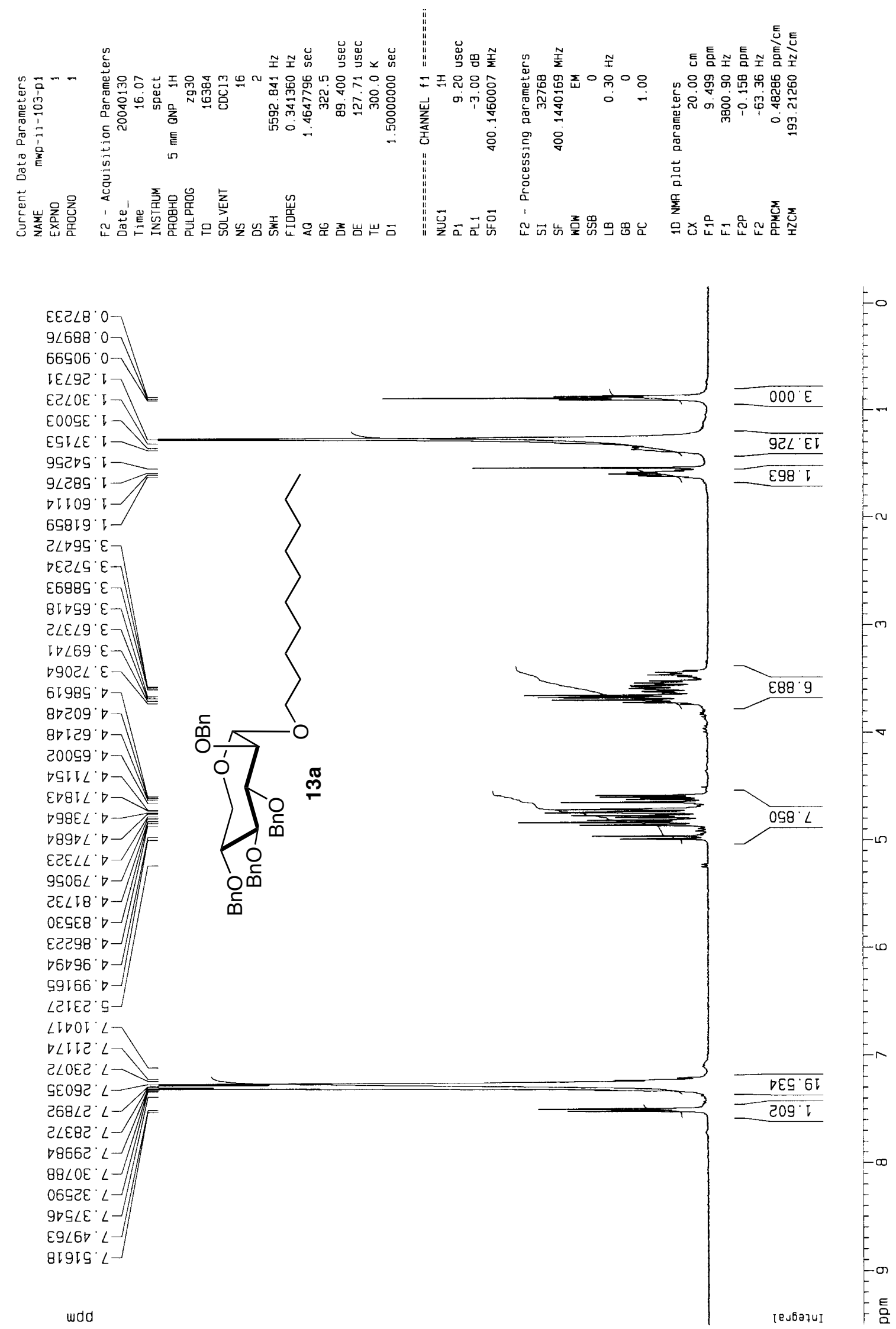

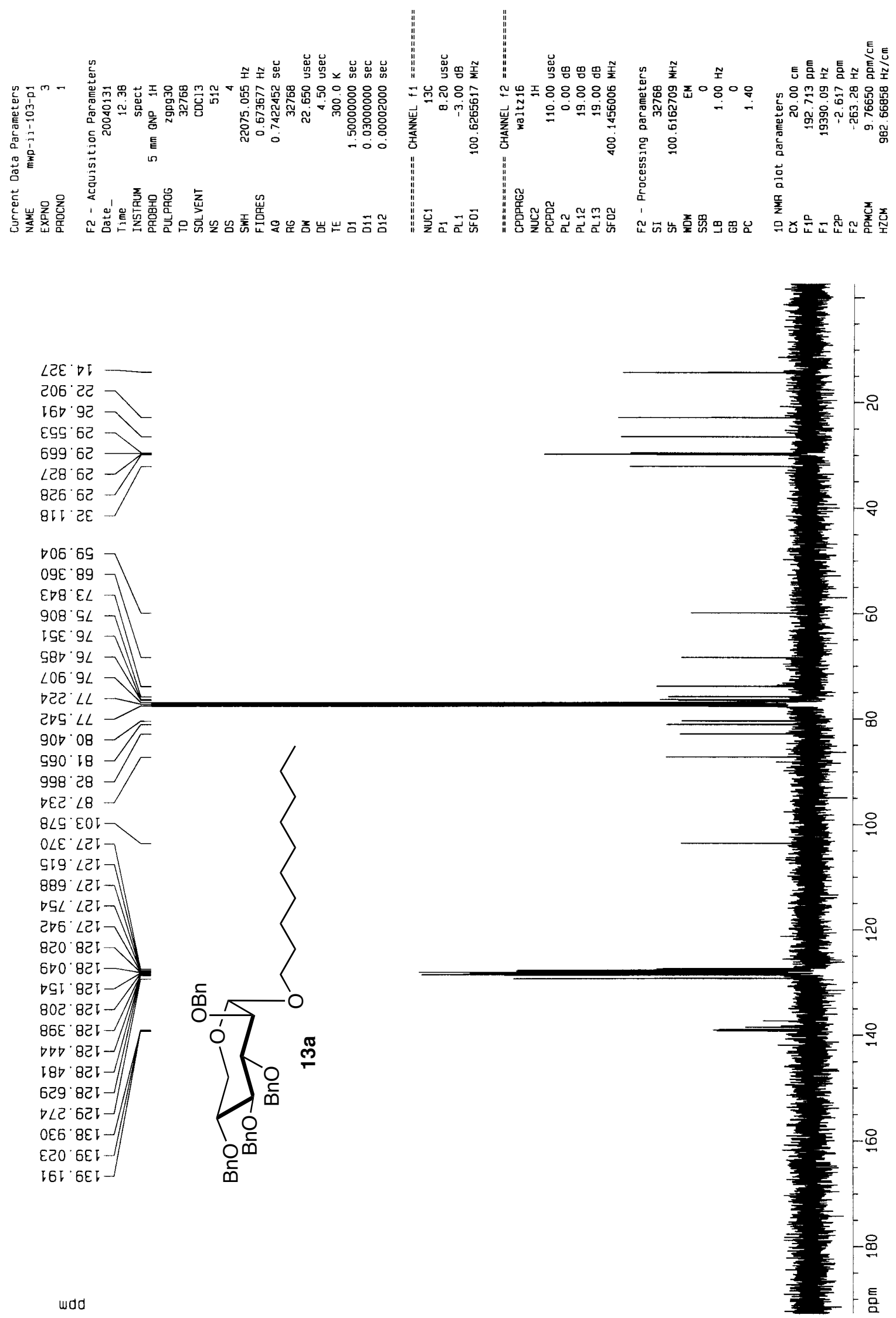

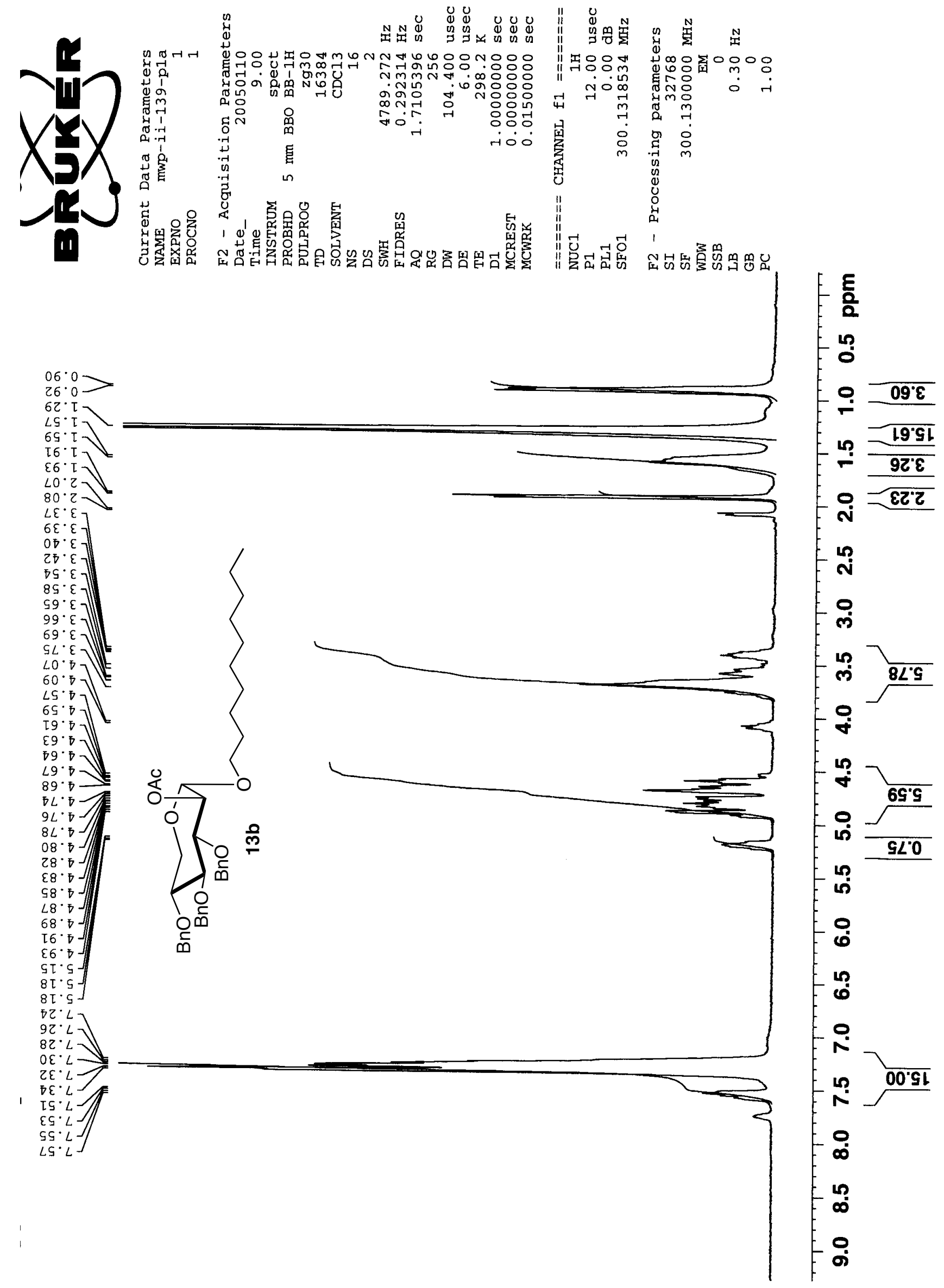

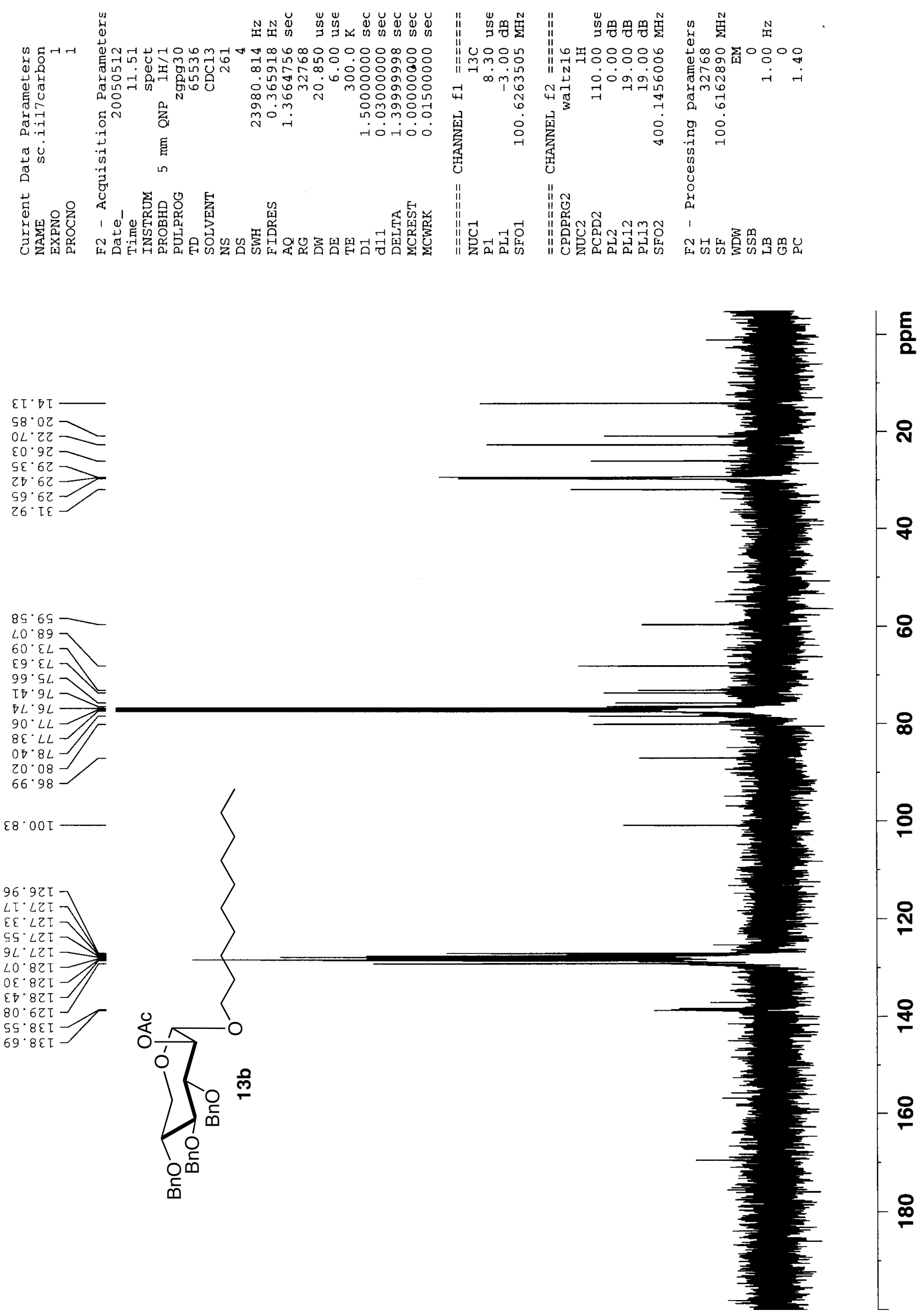

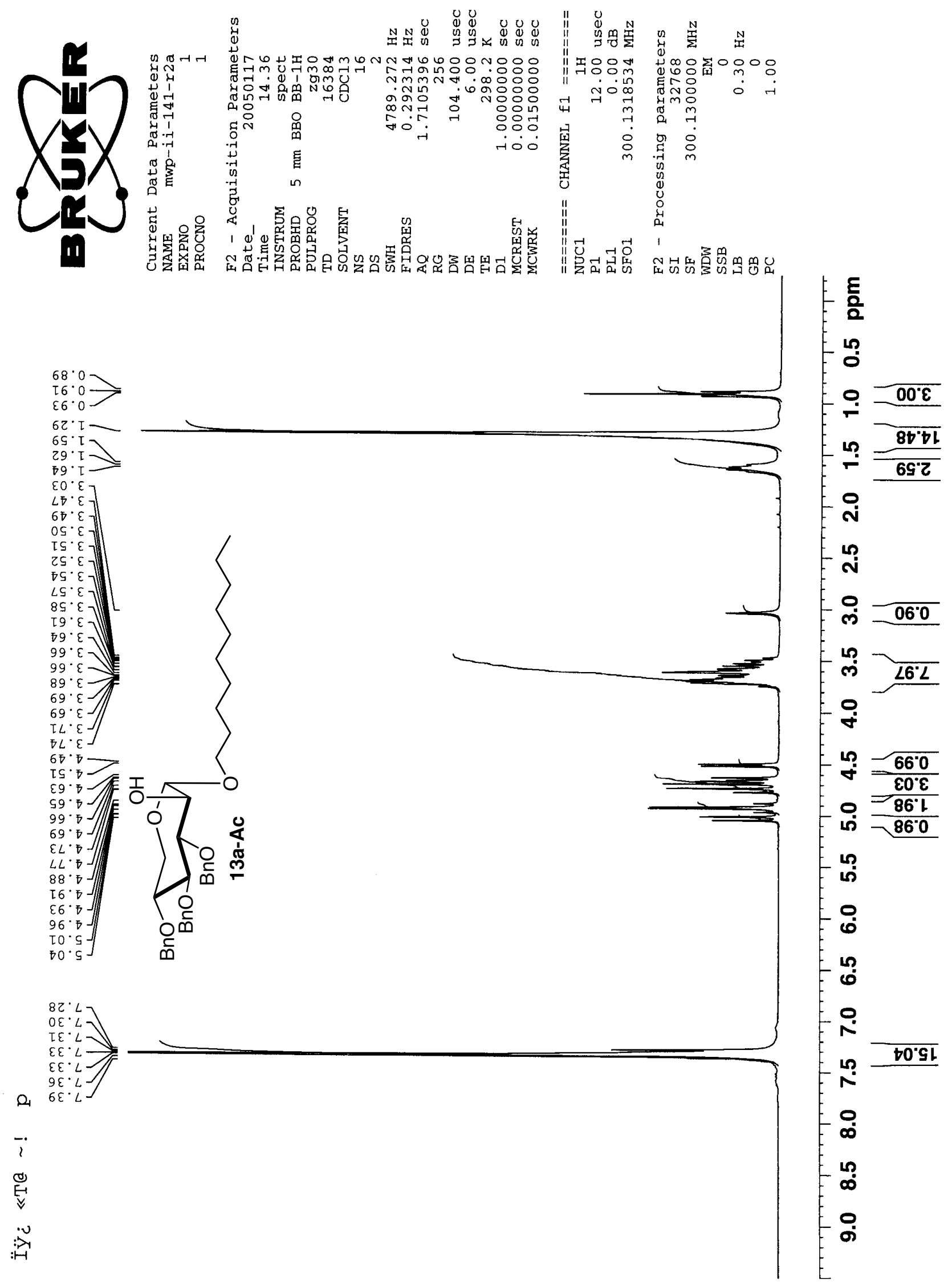


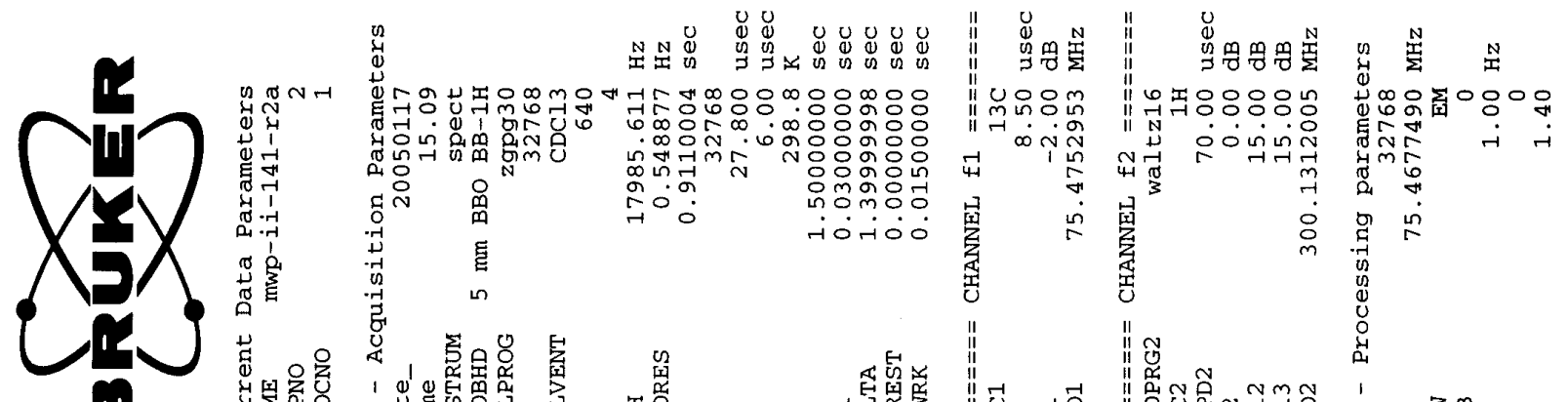

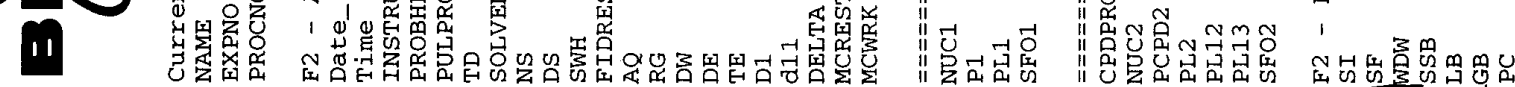

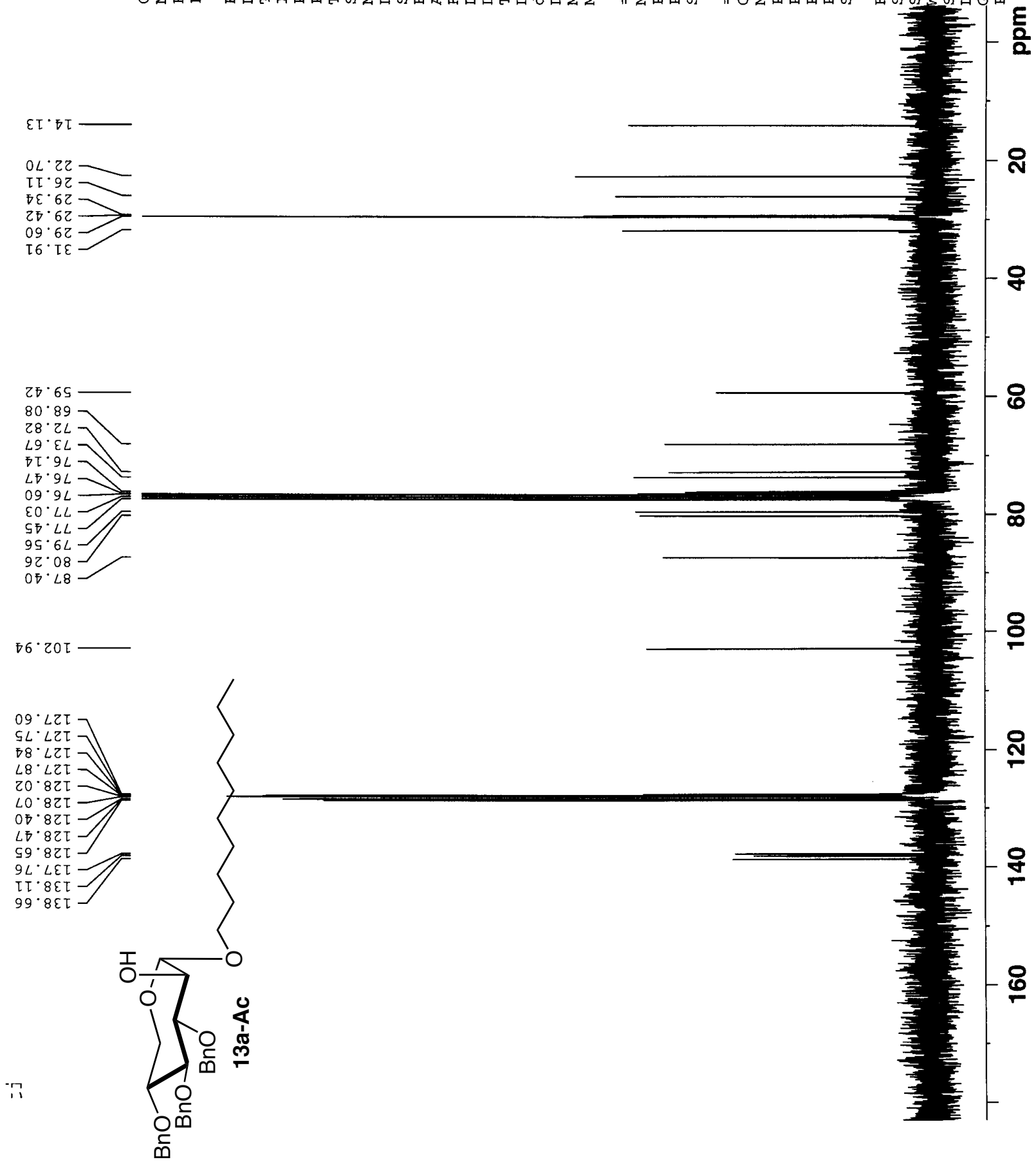



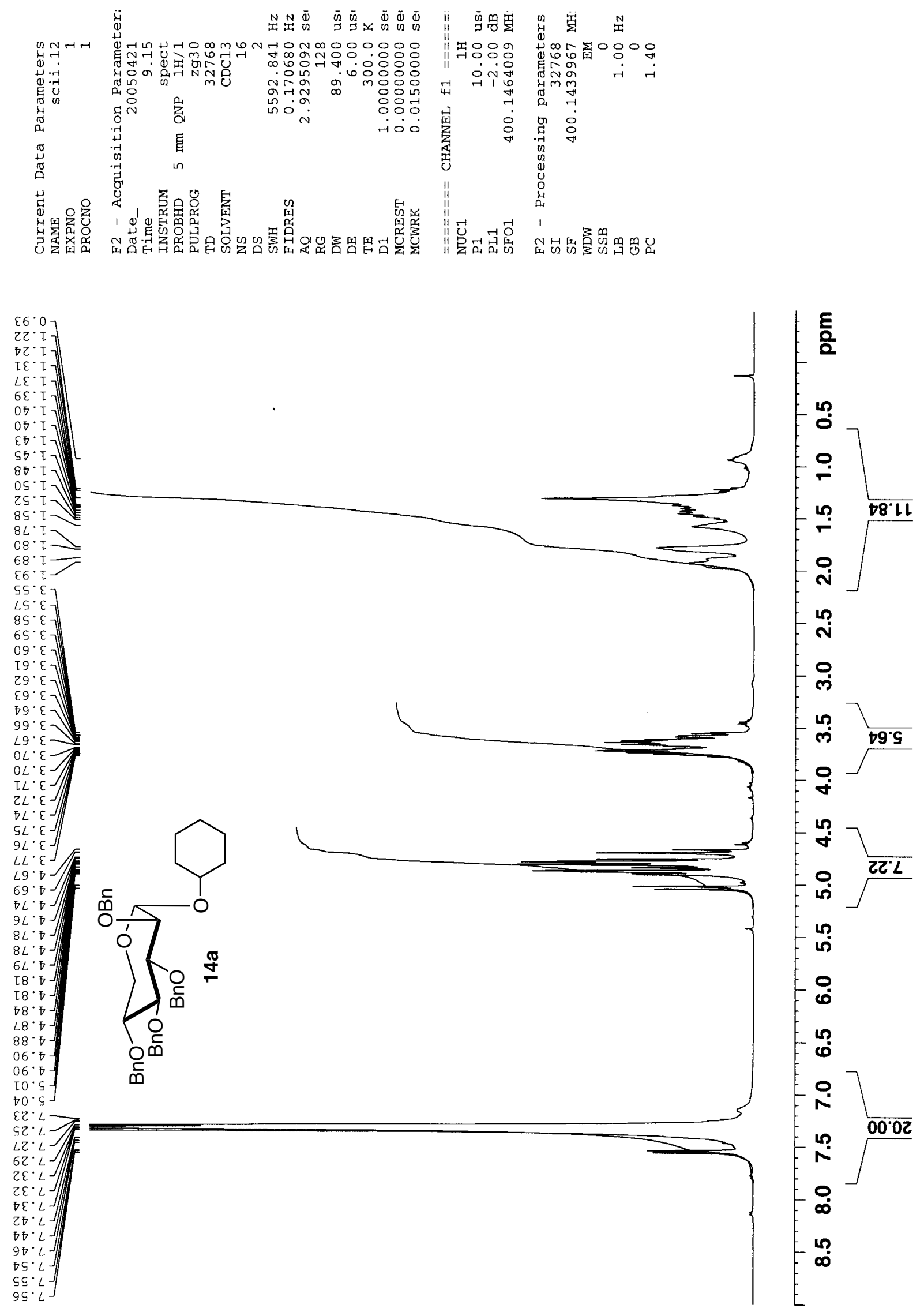

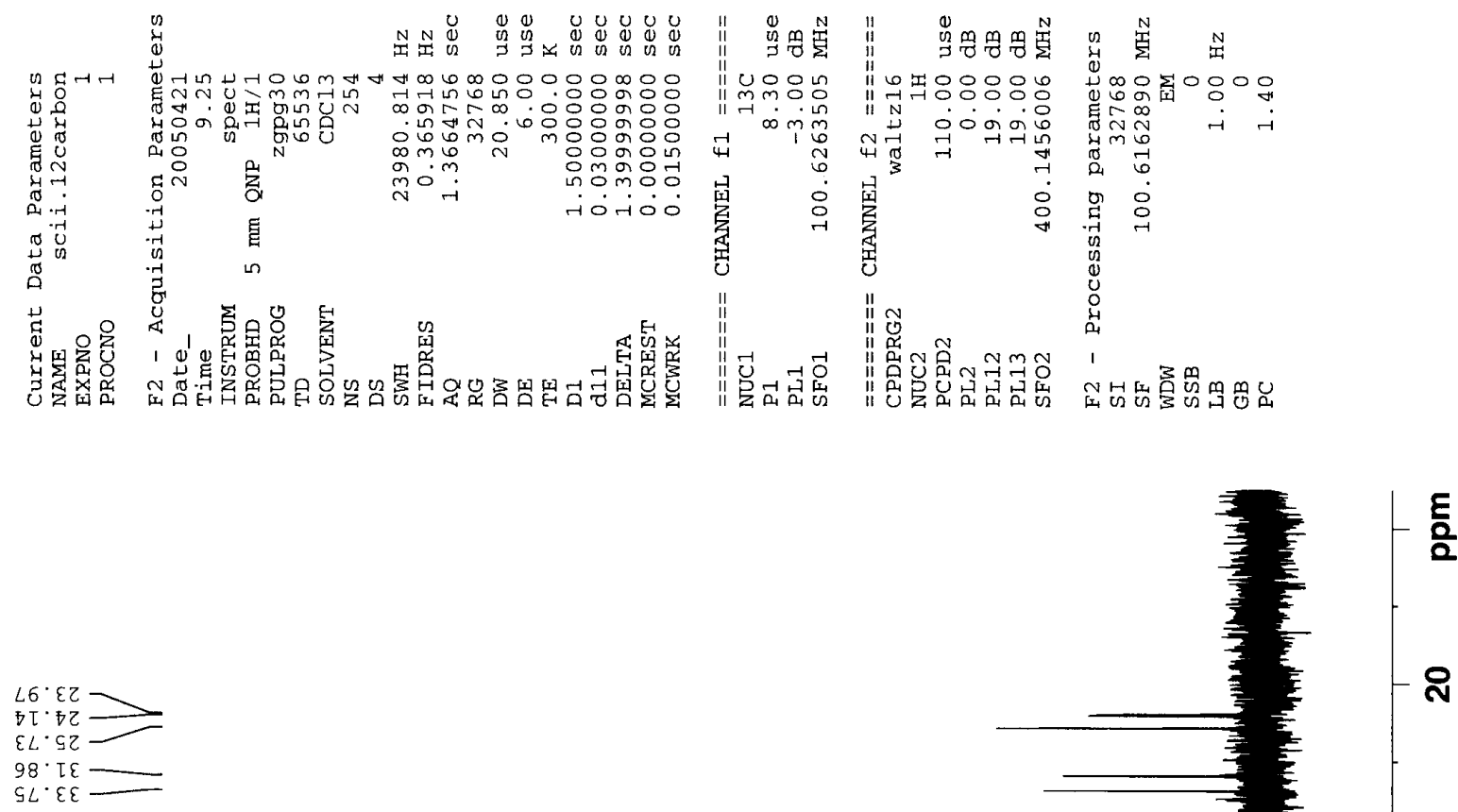

틍

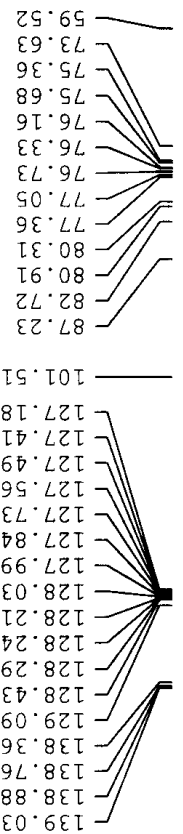

$88^{\circ} 8 \varepsilon[-]$
$\varepsilon 0 \cdot 6 \varepsilon[$
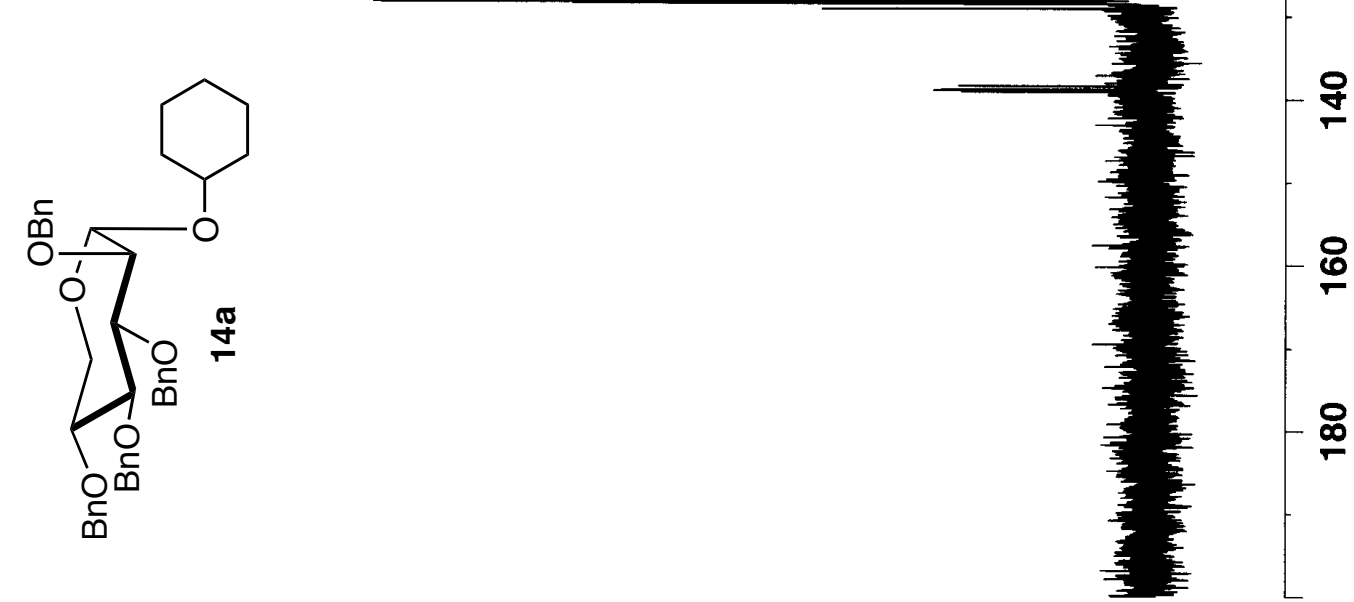

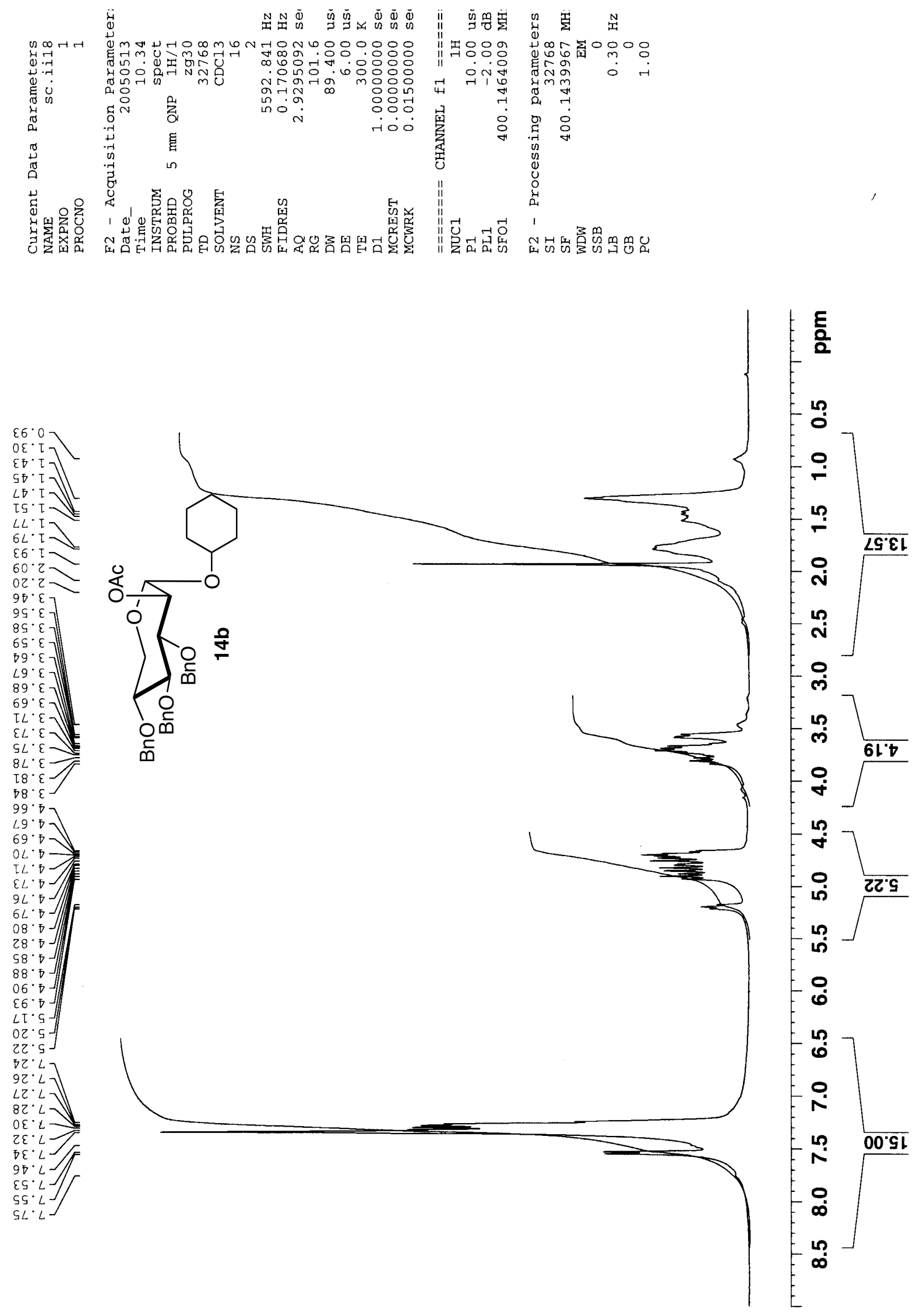

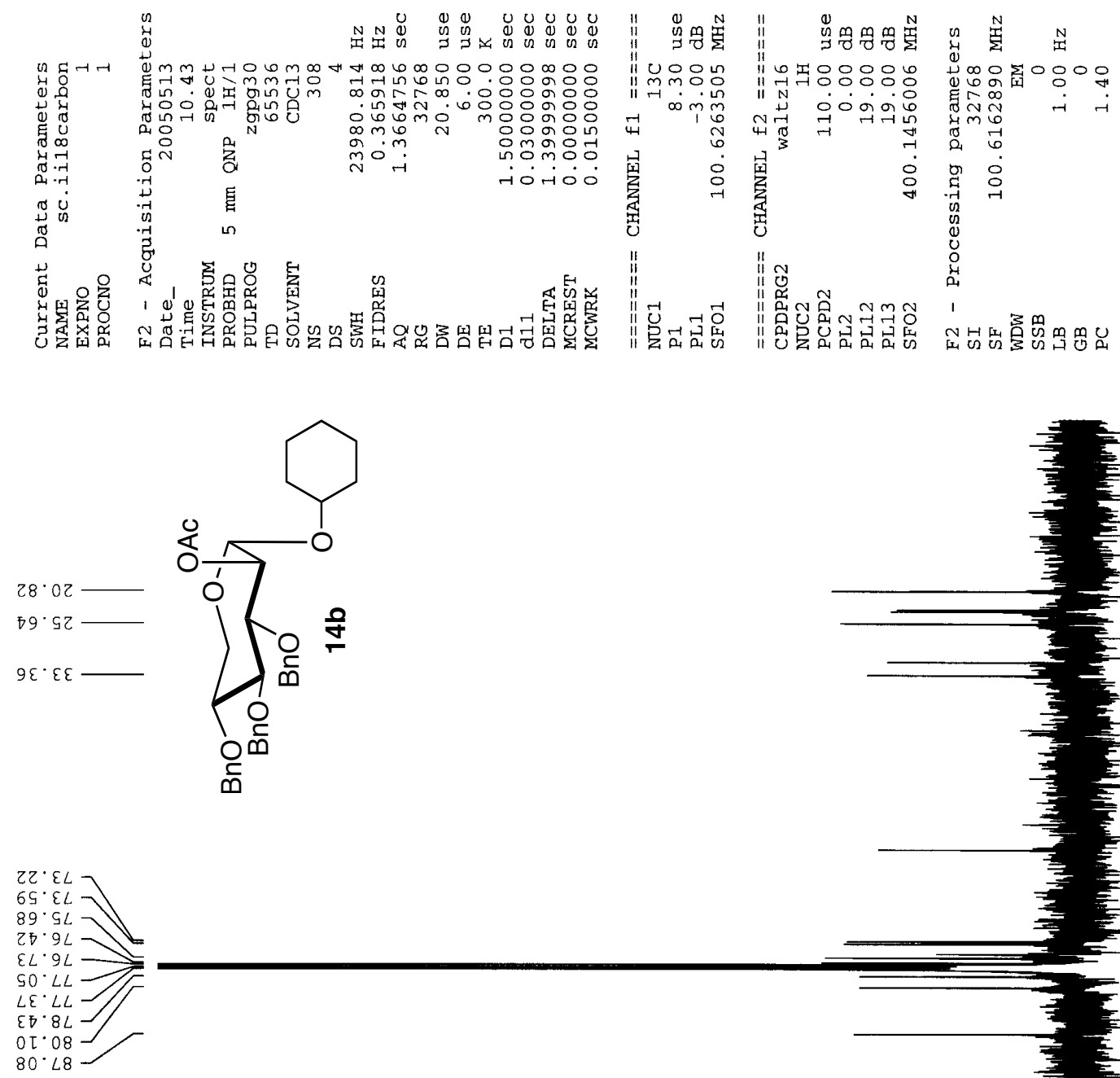

80.66
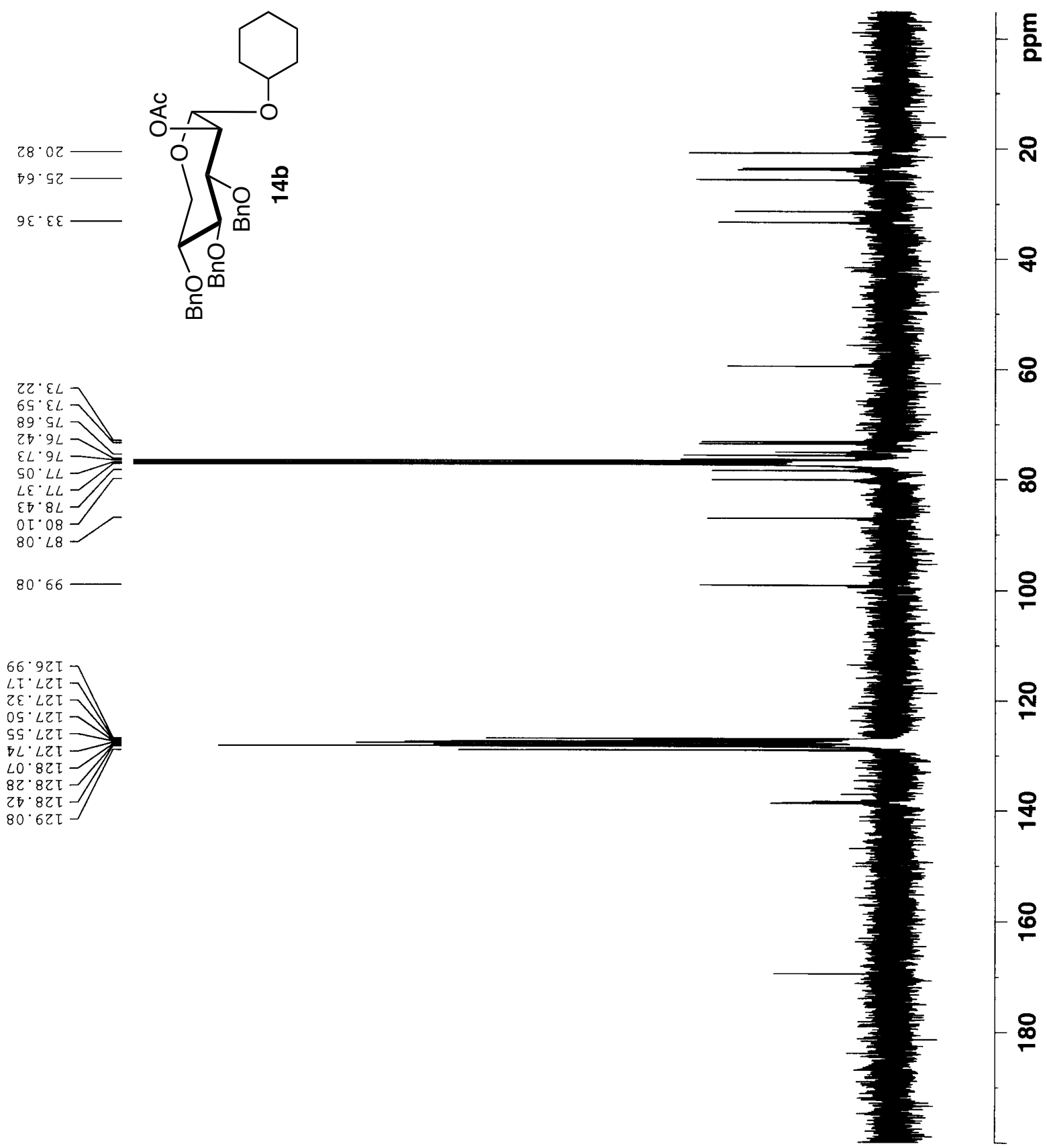

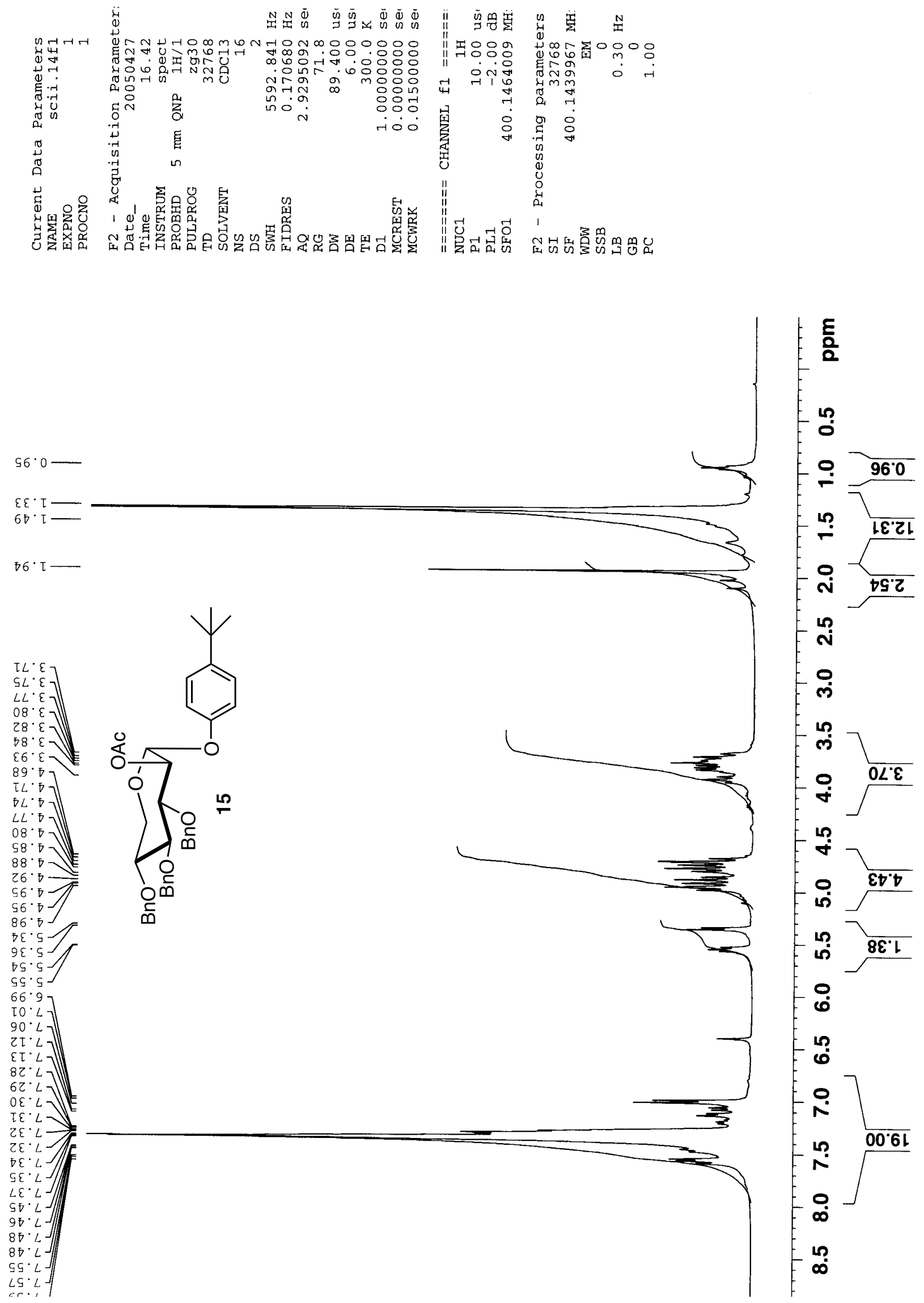

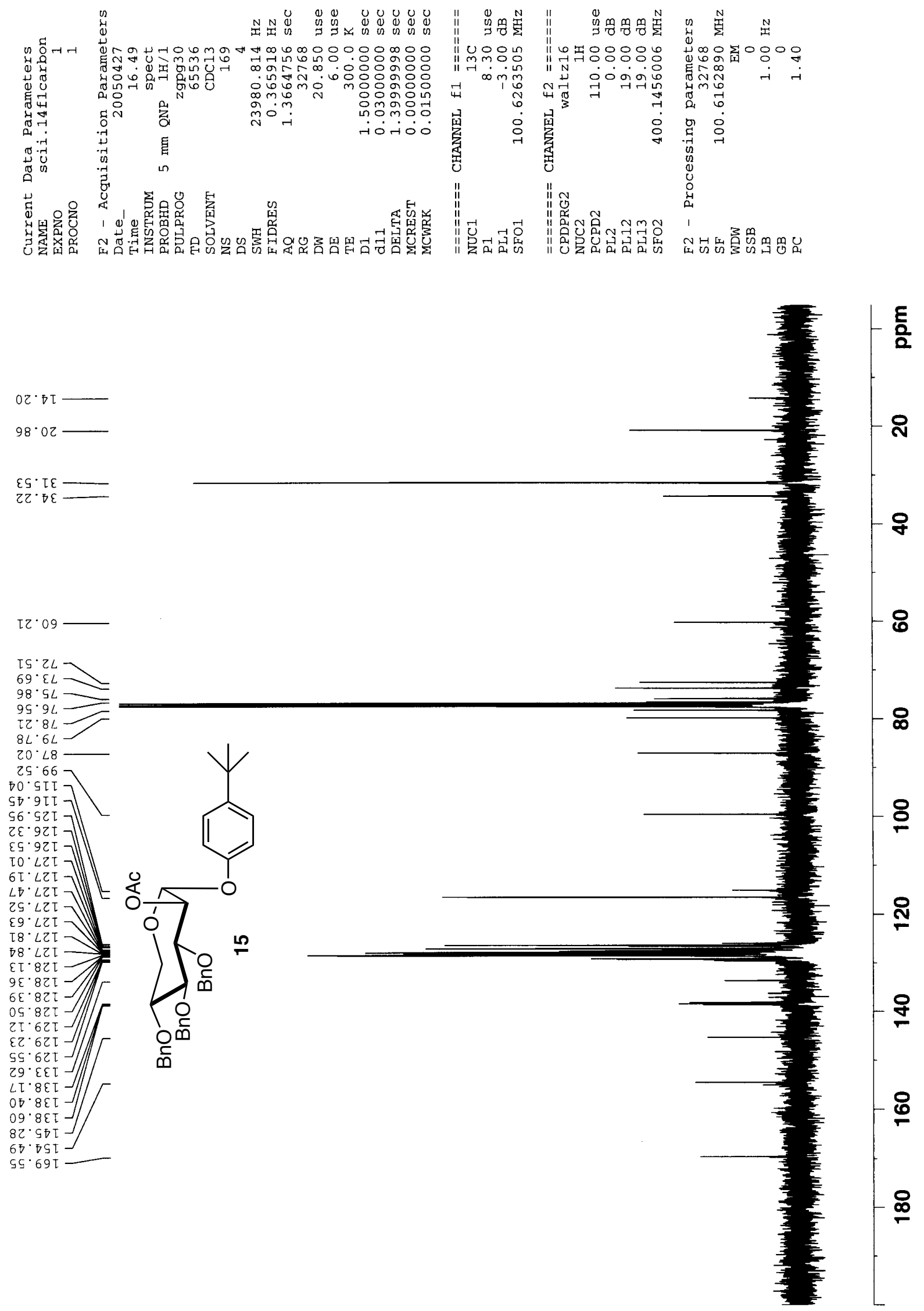

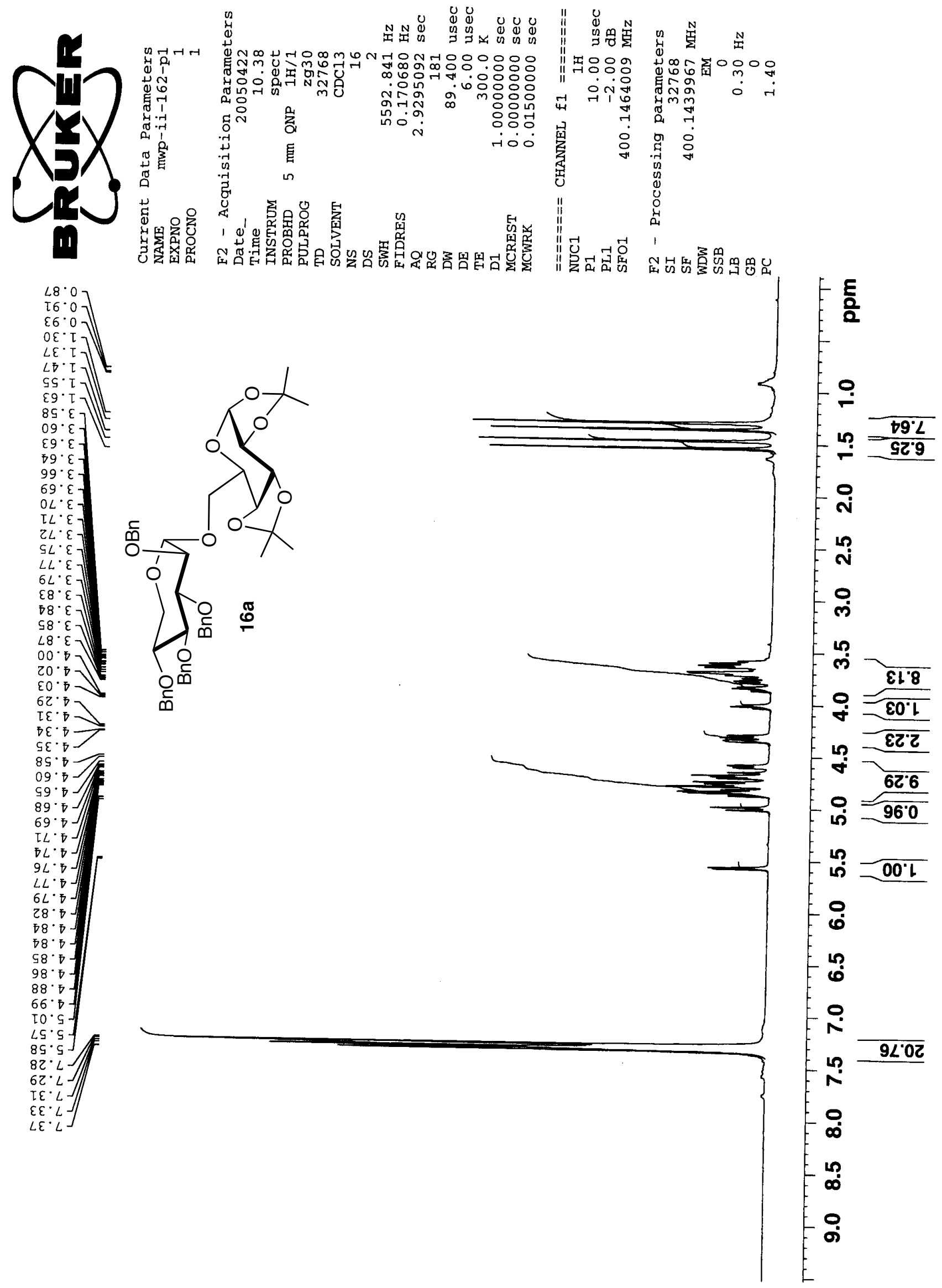

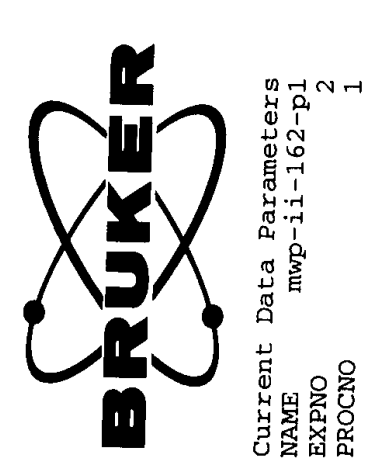

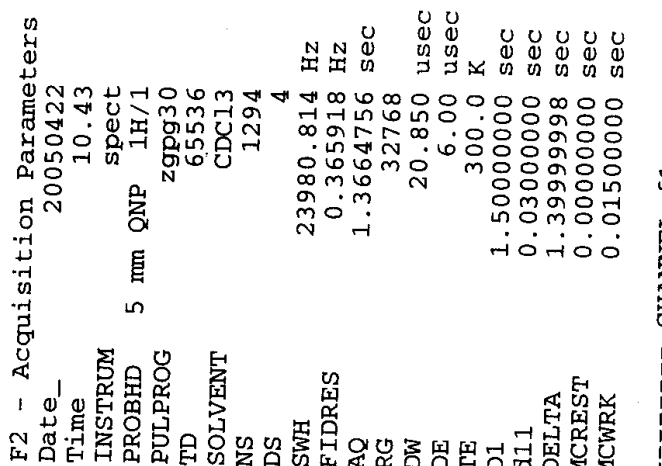
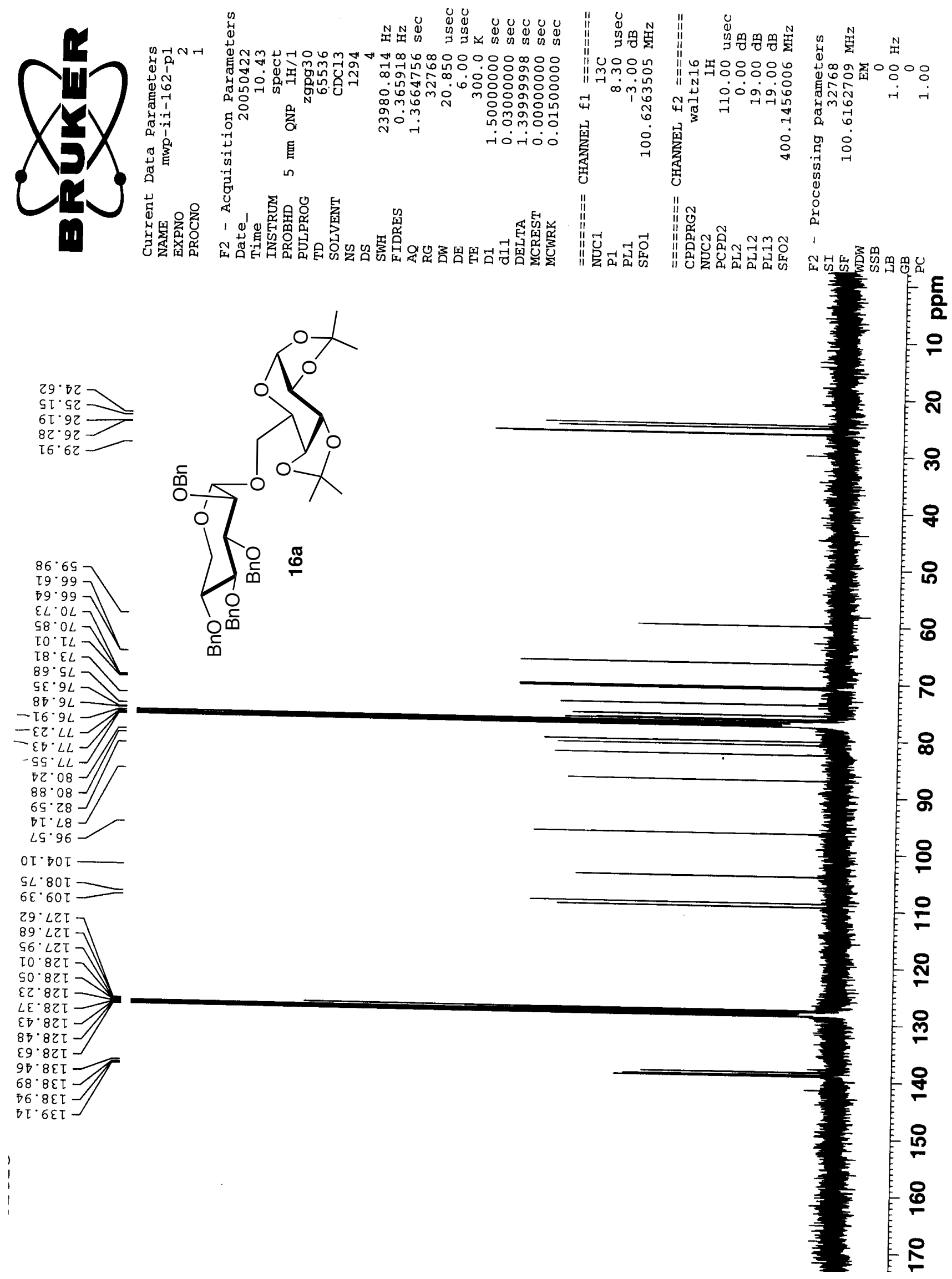

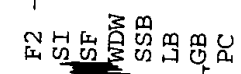



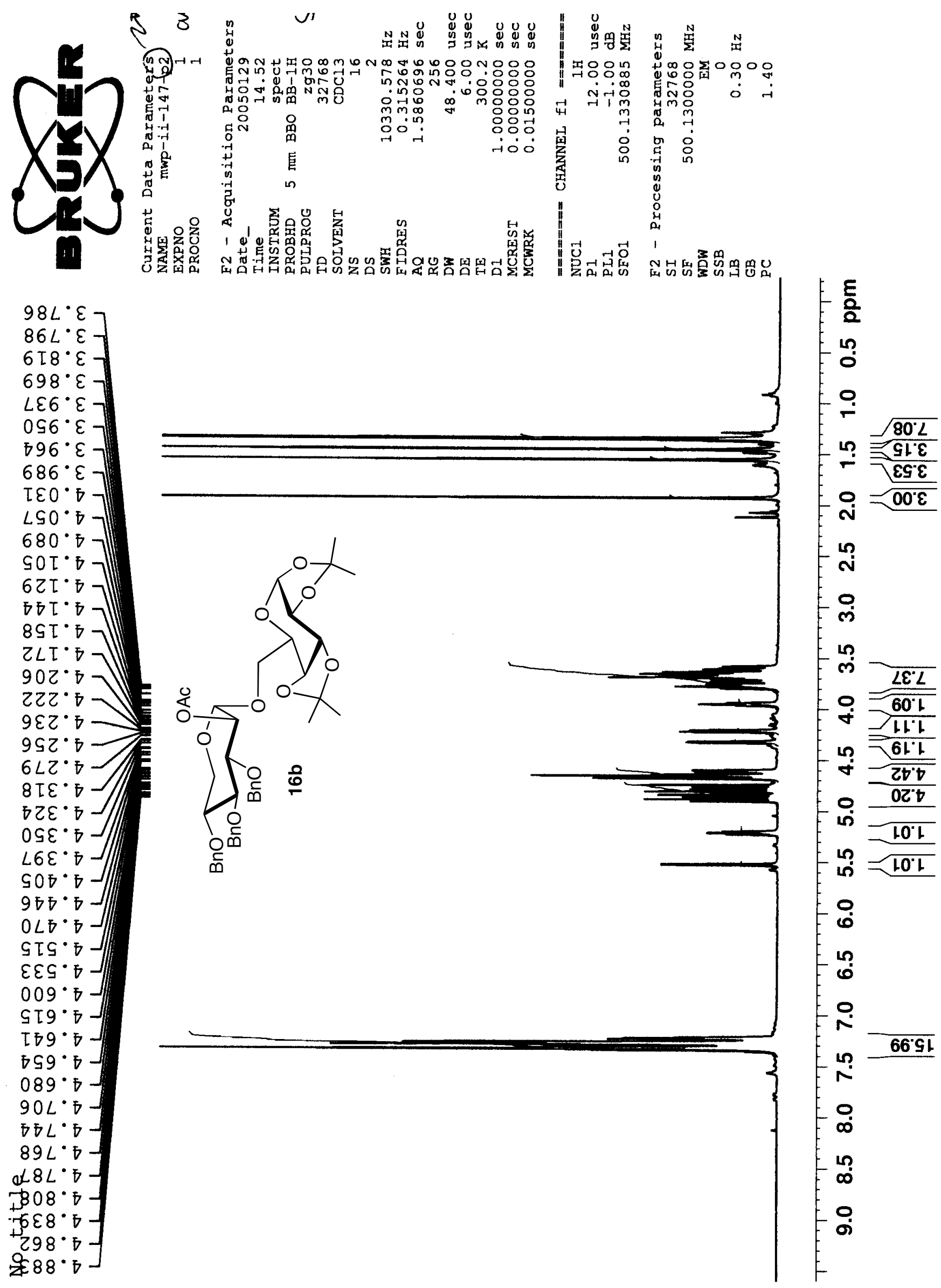

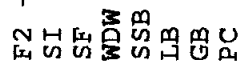



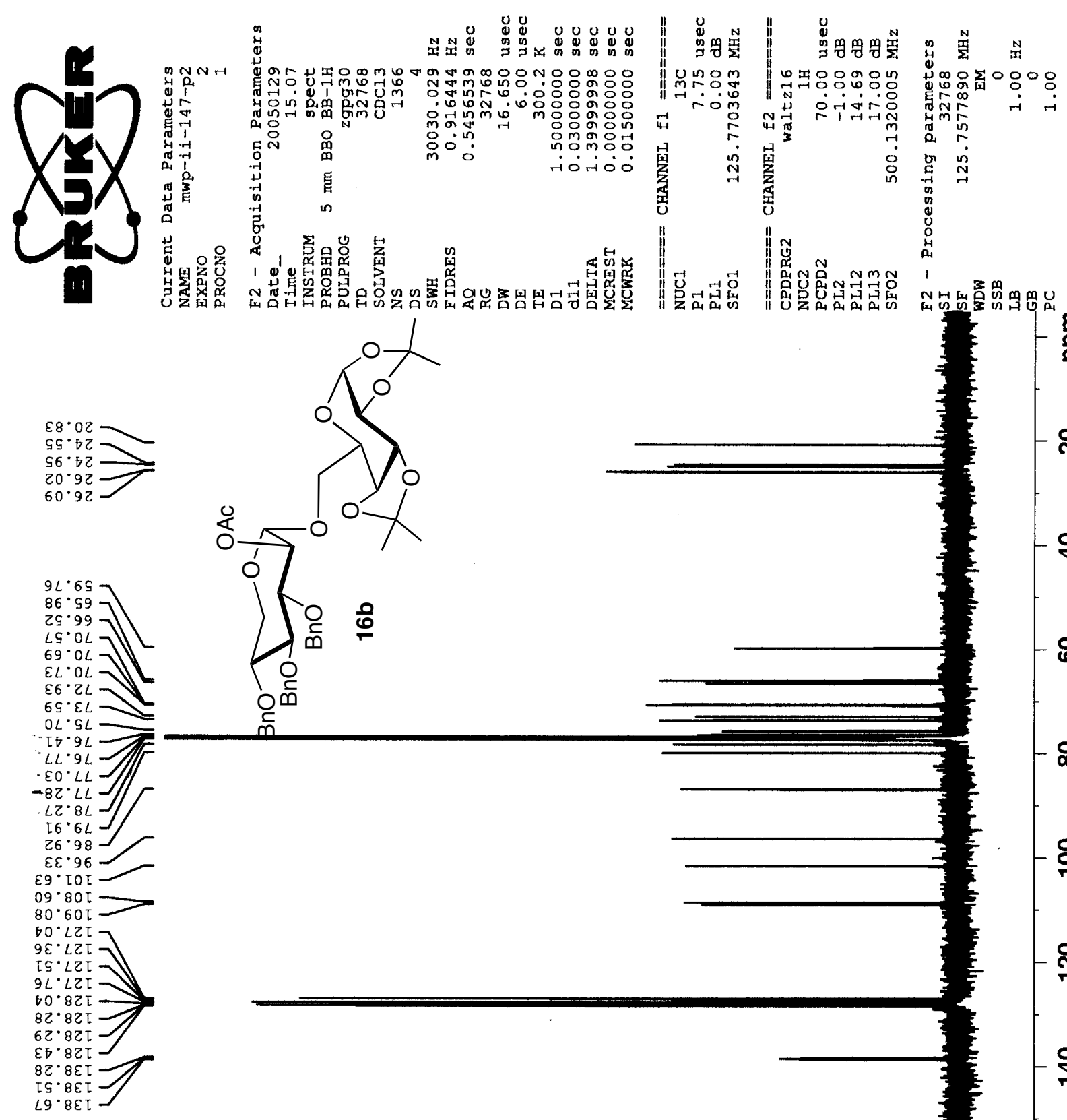

$\frac{\text { 을 }}{2}$

ล

우

8

ㅇ

\&

ํำ

온

๕

$65.69 t$

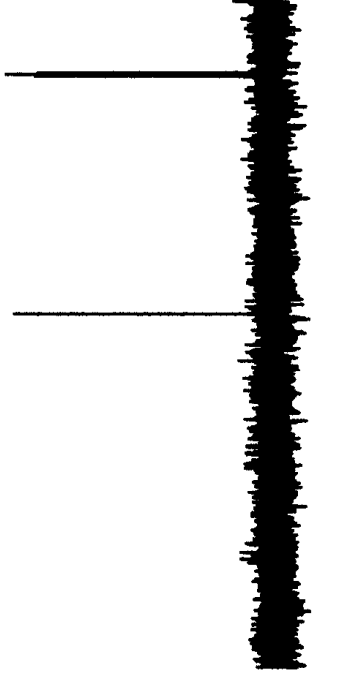

$\stackrel{\infty}{-}$

\& 


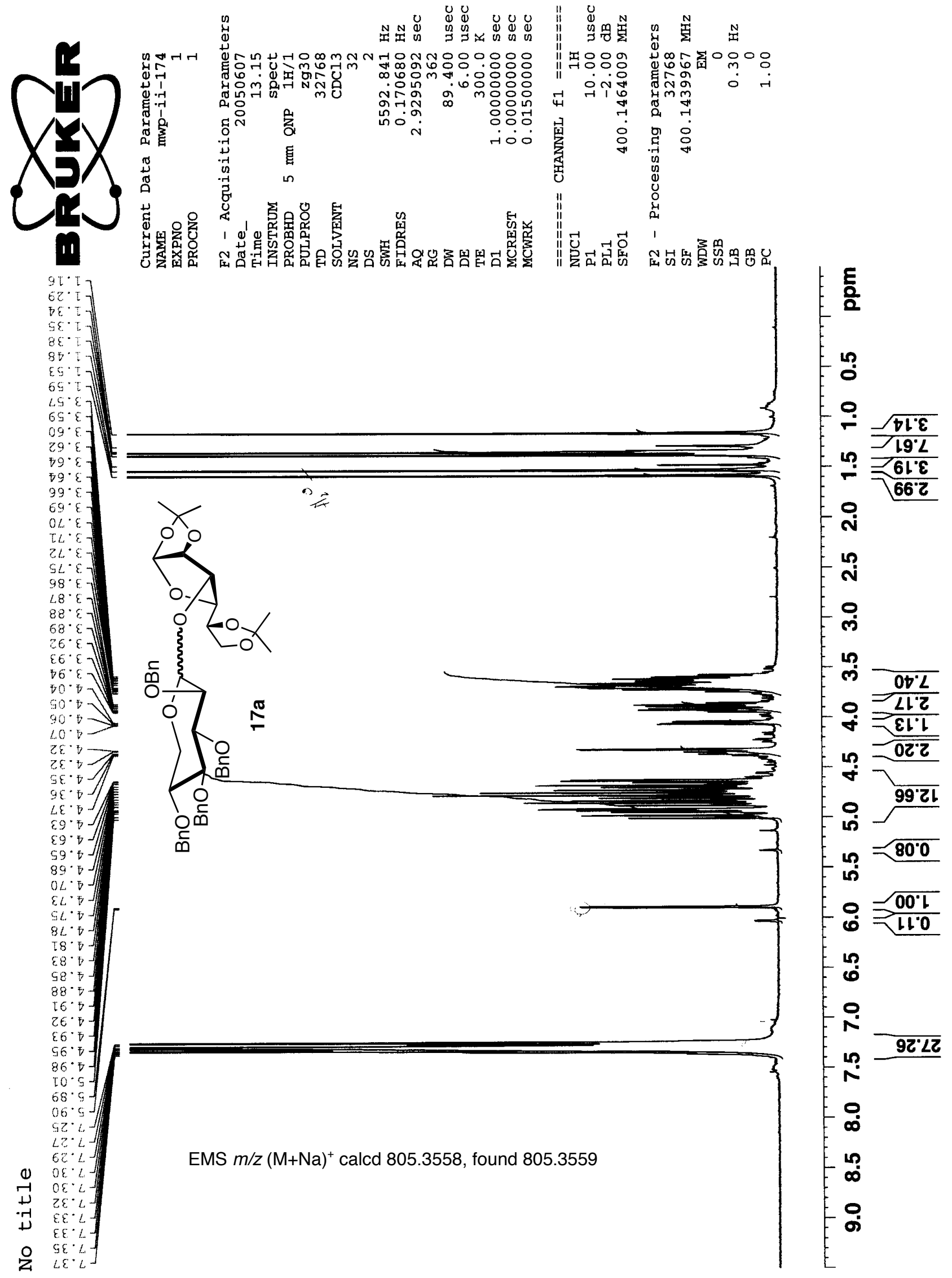




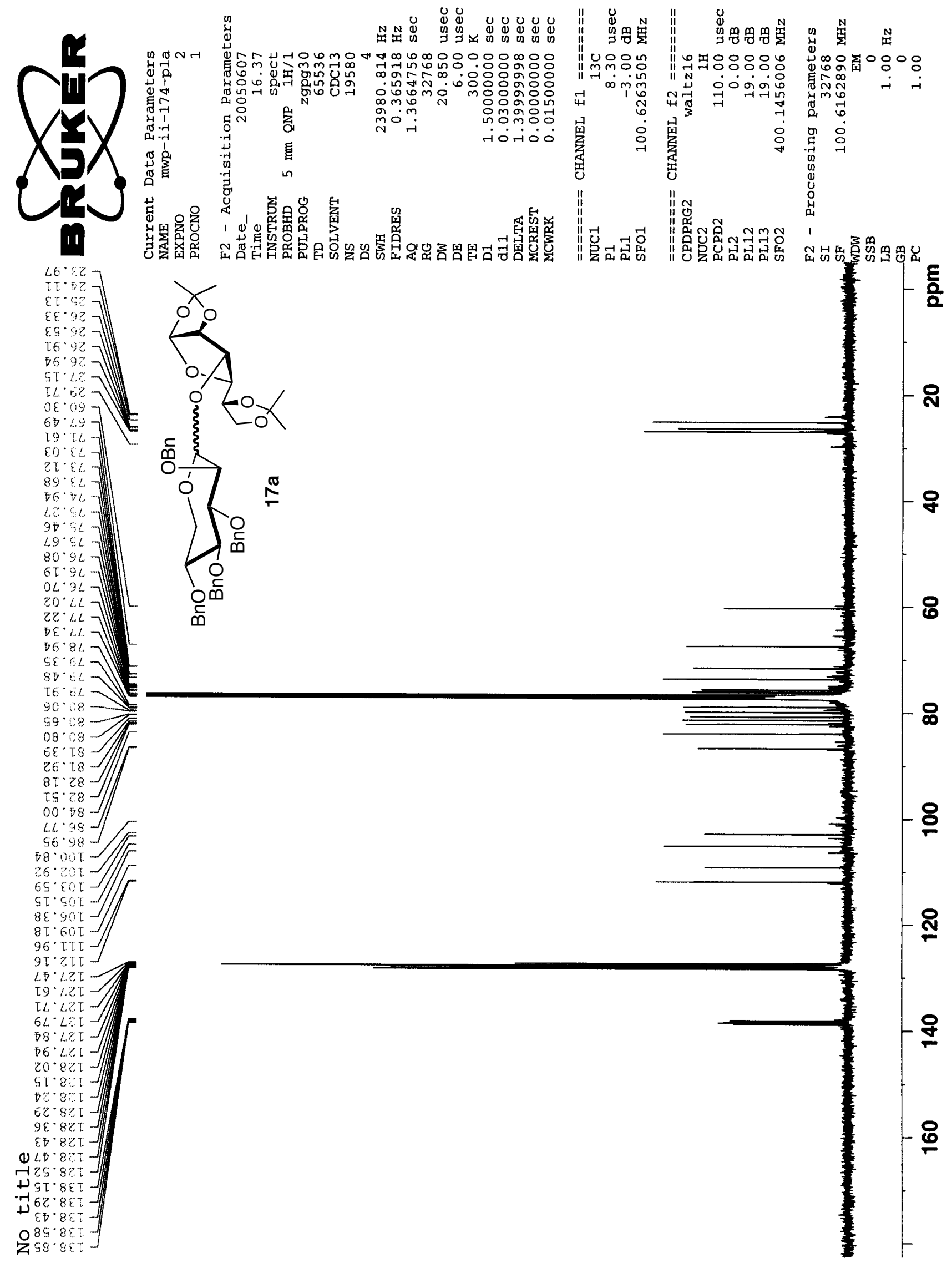




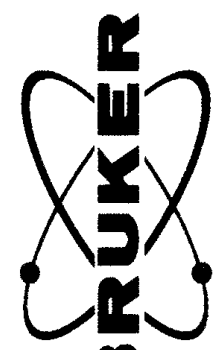

i1

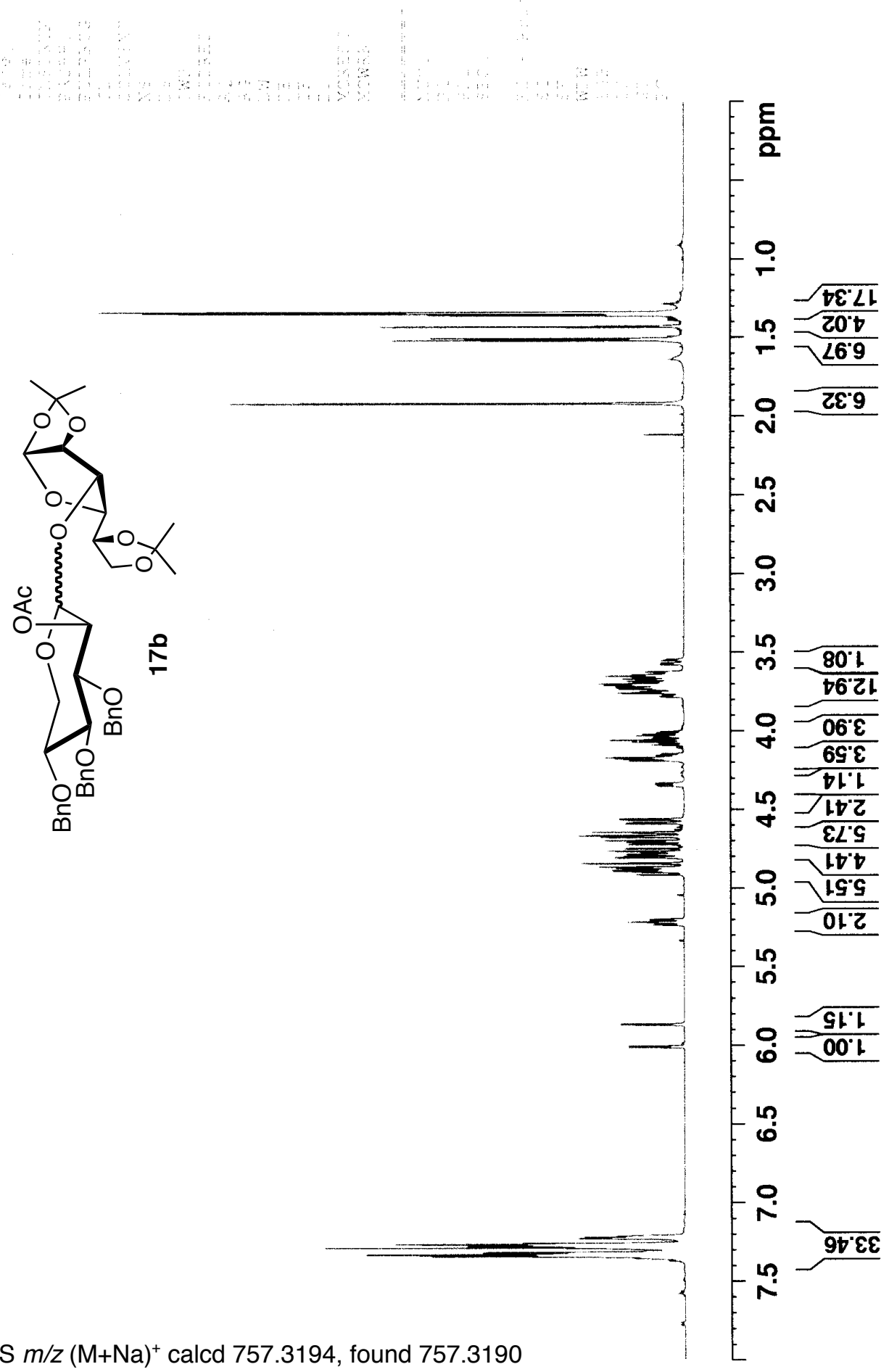



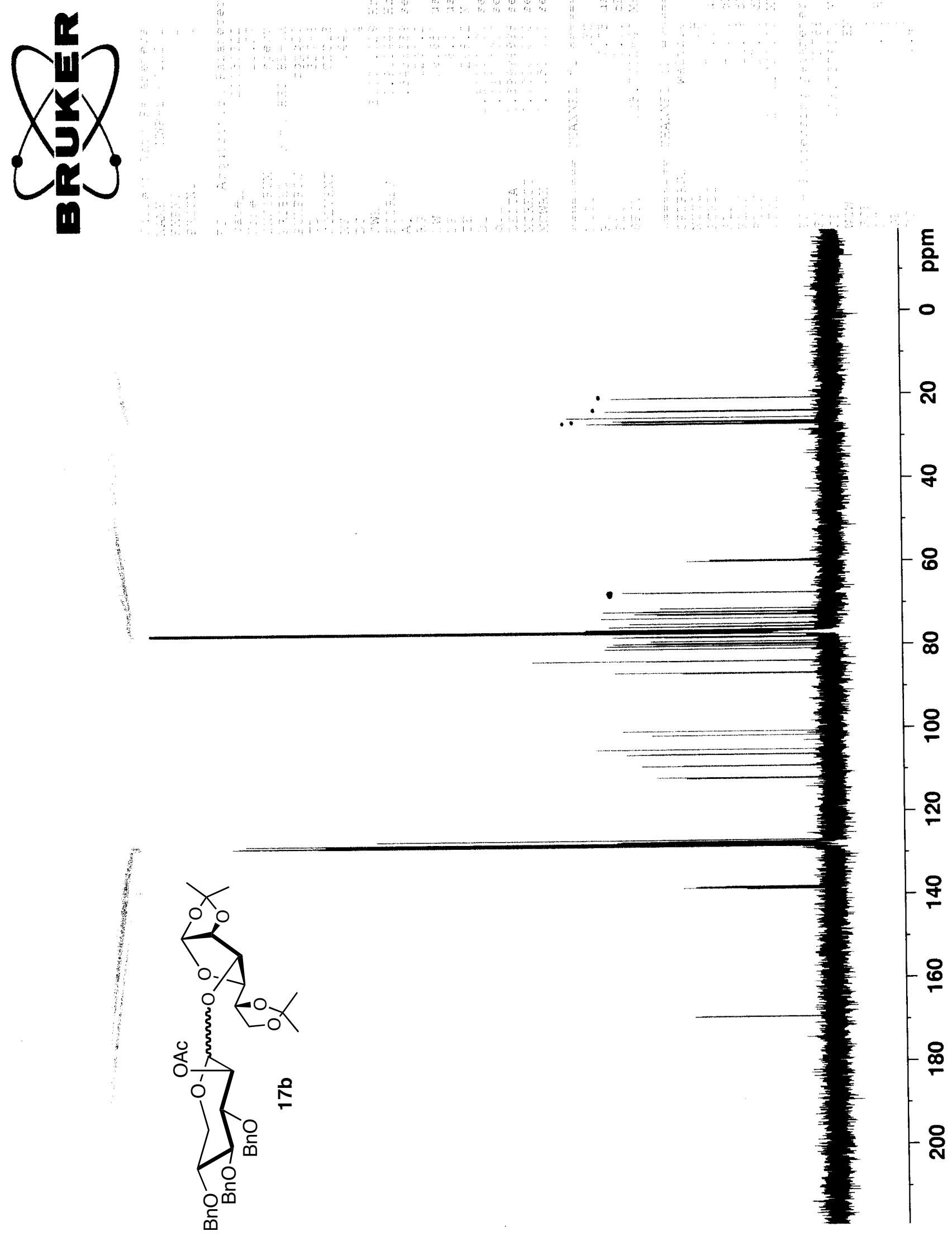


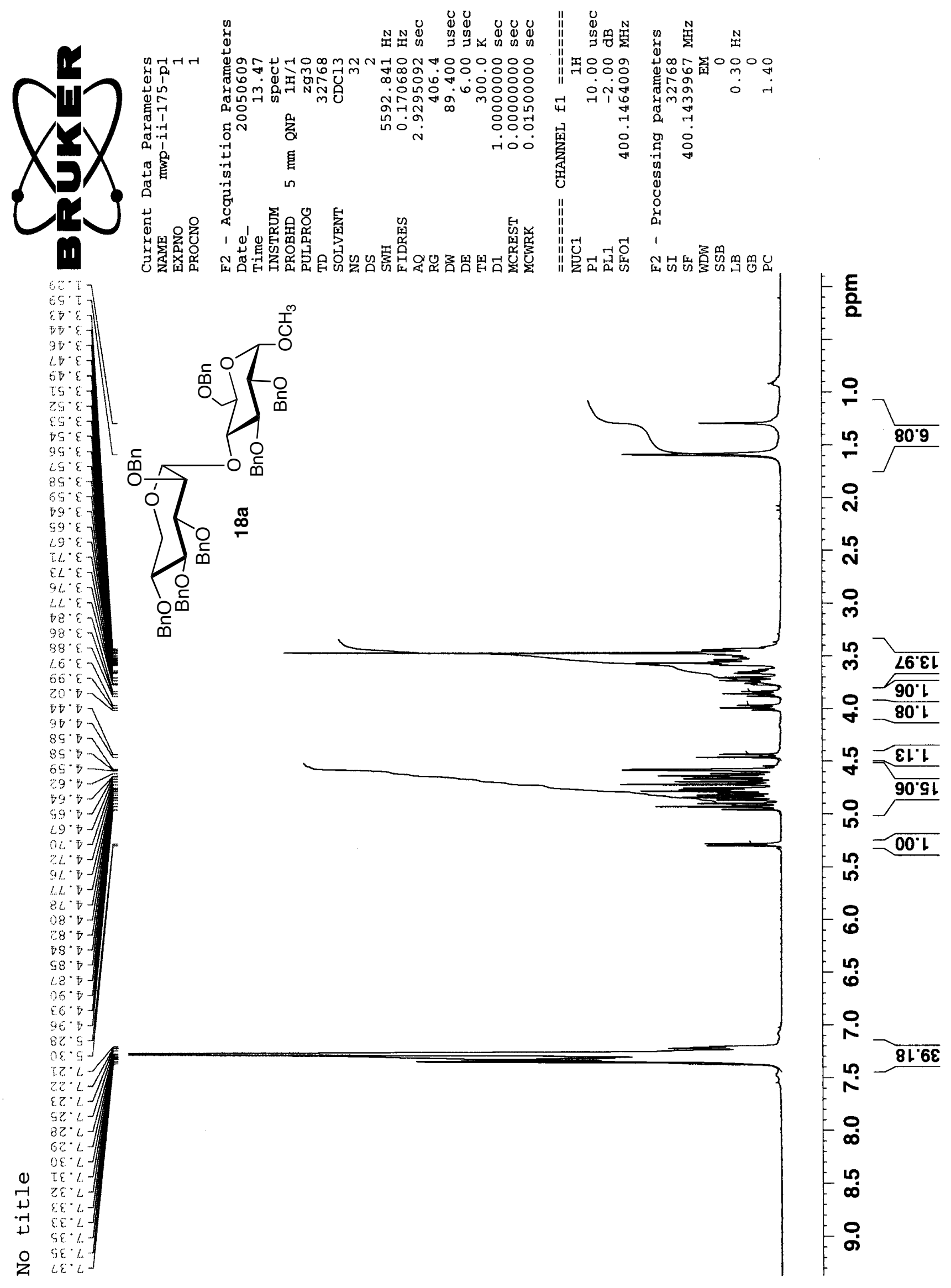



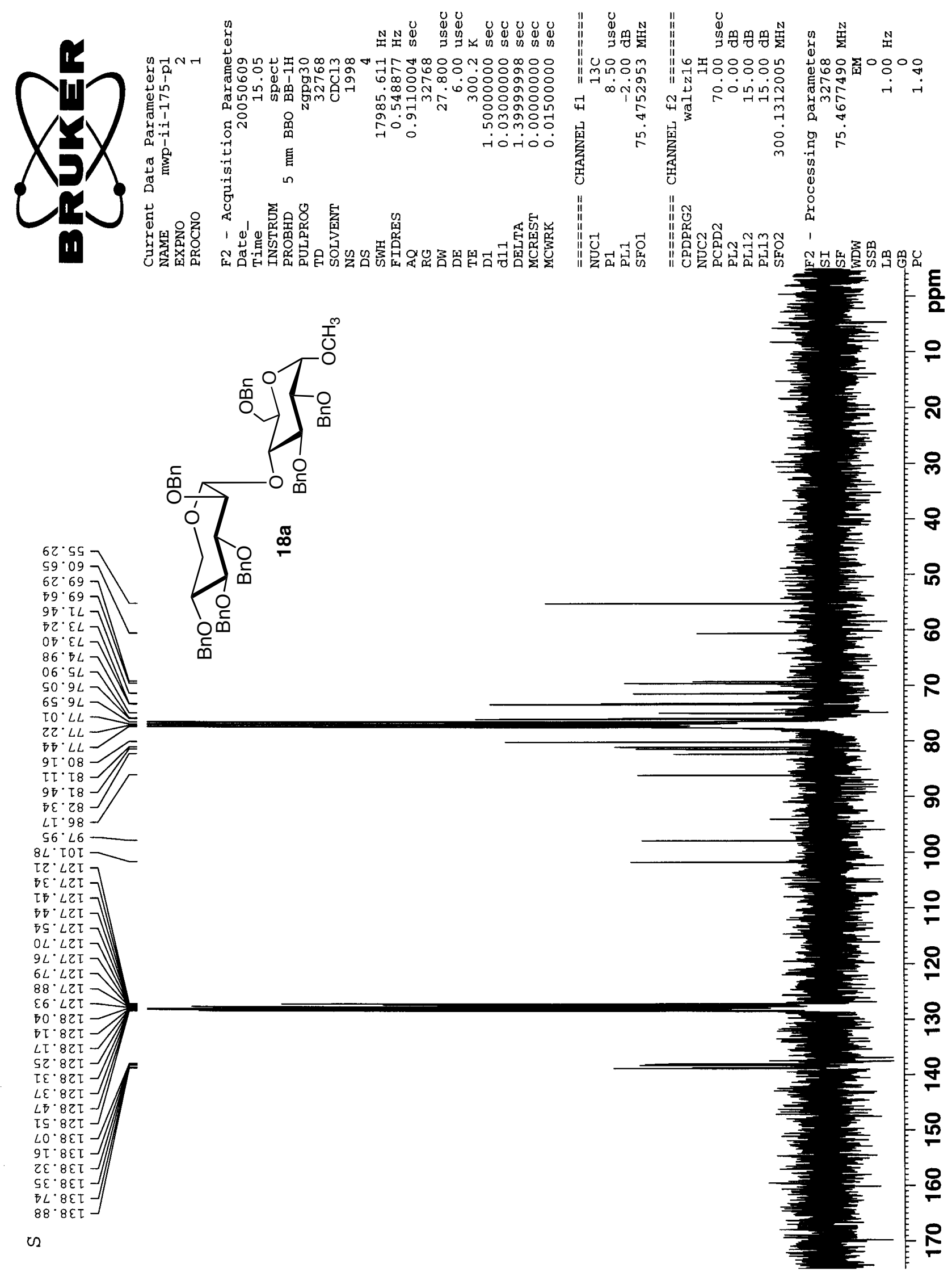

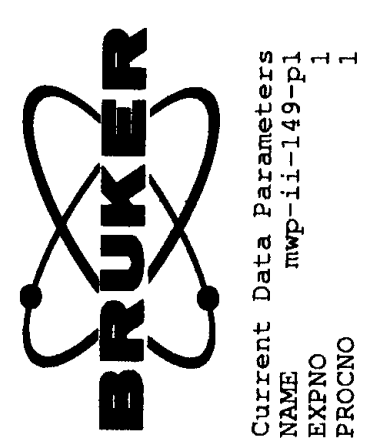

$906^{\circ} \varepsilon$ $\nabla 26^{\circ} \varepsilon$ 乙ธ6 $6^{\circ} \varepsilon$

$9 \angle 6^{\circ} \varepsilon$ $\nabla 66^{\circ} \varepsilon$ ZTO

8 โ $0^{\circ} \mathrm{O}$ $\angle E O^{\circ}$ $990^{\circ} \mathrm{B}$ $S \angle O^{\circ}$ $260^{\circ}$ $60 T^{\circ}$ $S D[\circ]$ ZS[. 0 โ9 T. $8 \angle T^{\circ}$ $892^{\circ}$ $8 \varepsilon \varepsilon \cdot \square$ $8 \nabla \varepsilon^{\circ} \nabla$ $\varepsilon L \varepsilon^{\circ} \circ$ $\angle O \sigma^{\circ}$
$\angle Z \sigma^{\circ}$
$\angle 9 \sigma^{\circ}$

I6 $0^{\circ} \circ$ OZS $2 \angle S^{\circ} \circ$ S8. 0 $009^{\circ} \mathrm{\nabla}$ $529^{\circ} \mathrm{\sigma}$ $059^{\circ} \square$ $289^{\circ} \circ$ $8 Z L^{\circ} \circ$ $Z \forall L \cdot G$ $\varepsilon S L \cdot \sigma-$ $99 L^{\circ} \sigma$ $S L L^{\circ} \sigma$ 2 โ $8^{\circ}$ 万 ब2 $28^{\circ} \circ-$ $-898^{\circ} \circ-$ $-5<8^{\circ} \cdot 1-1$ 万68. ₹

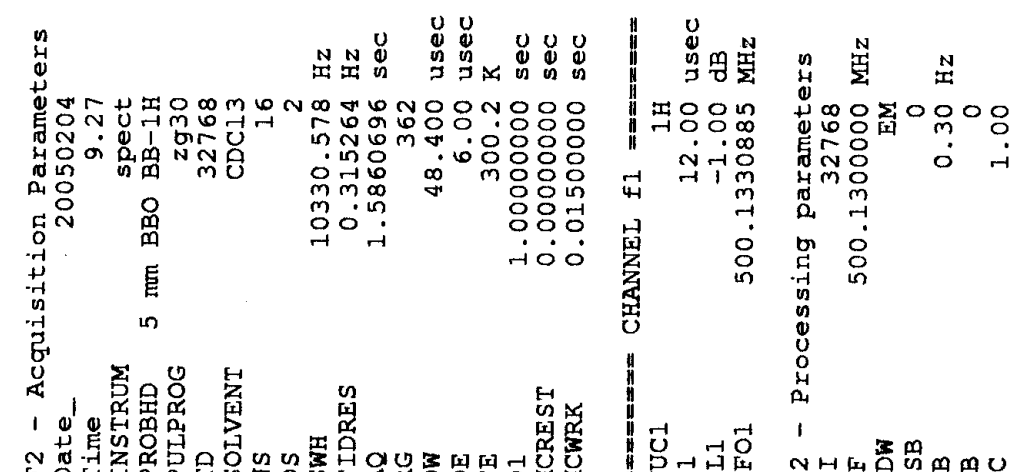

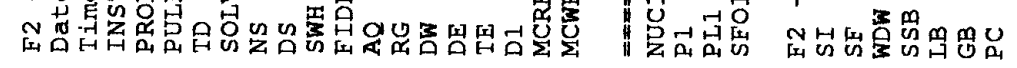




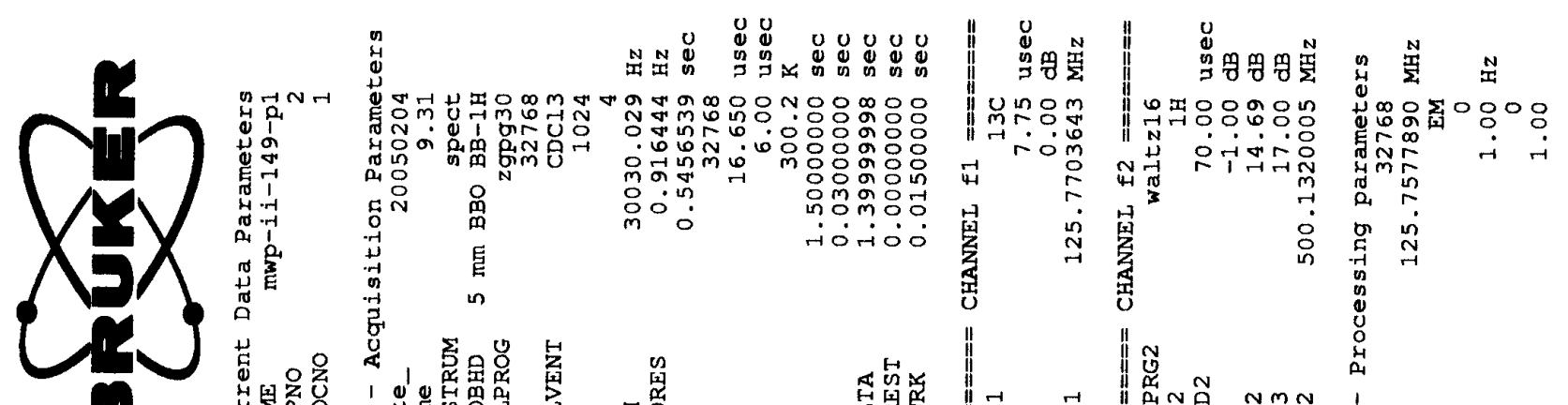

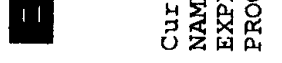

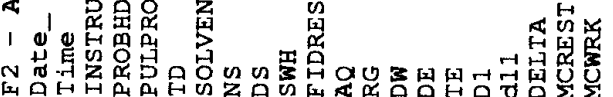

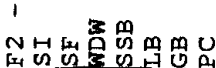

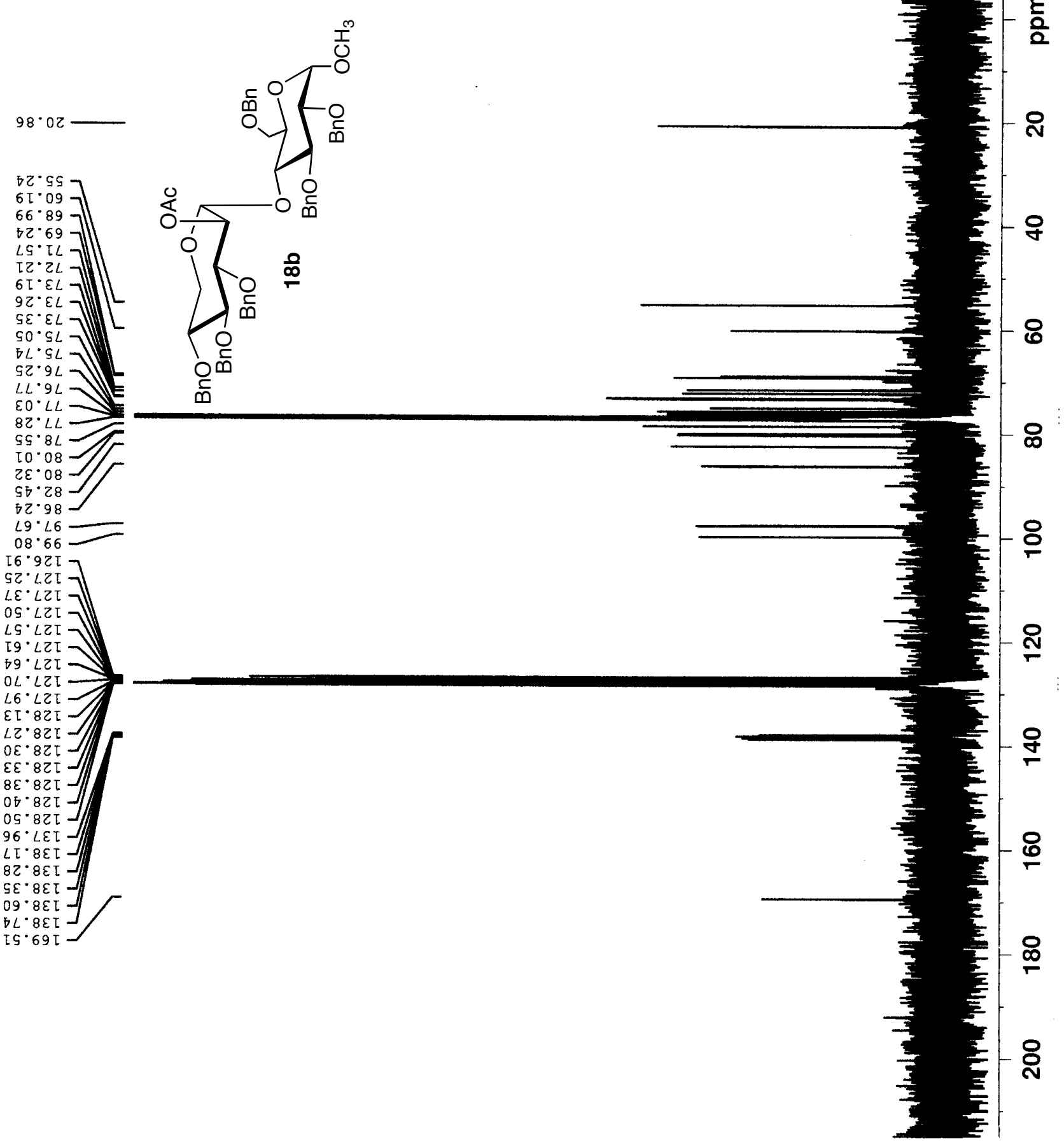



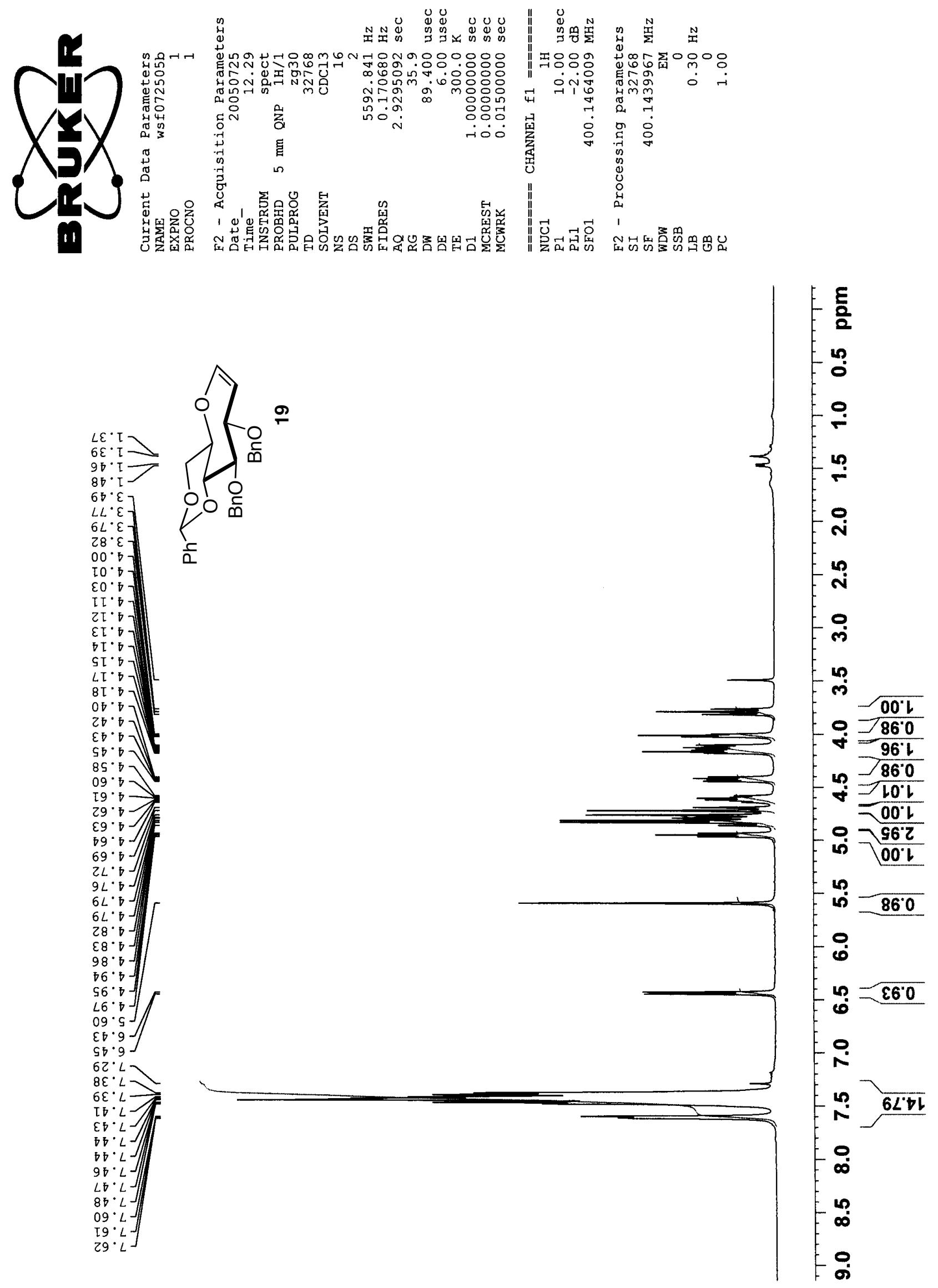

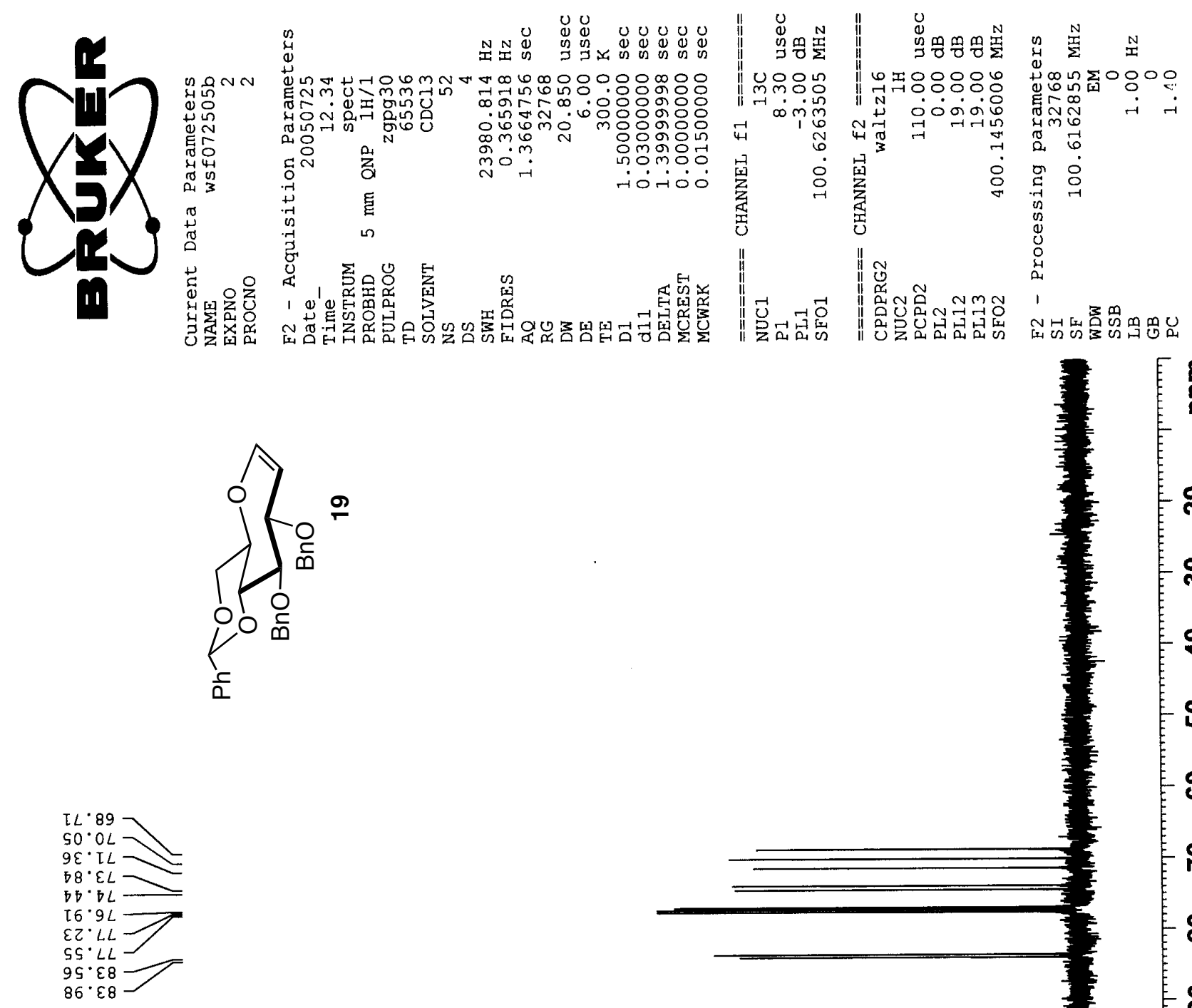

틍

$6 L \cdot 00 \mathrm{~T}$

$\llcorner\varepsilon$ SOT
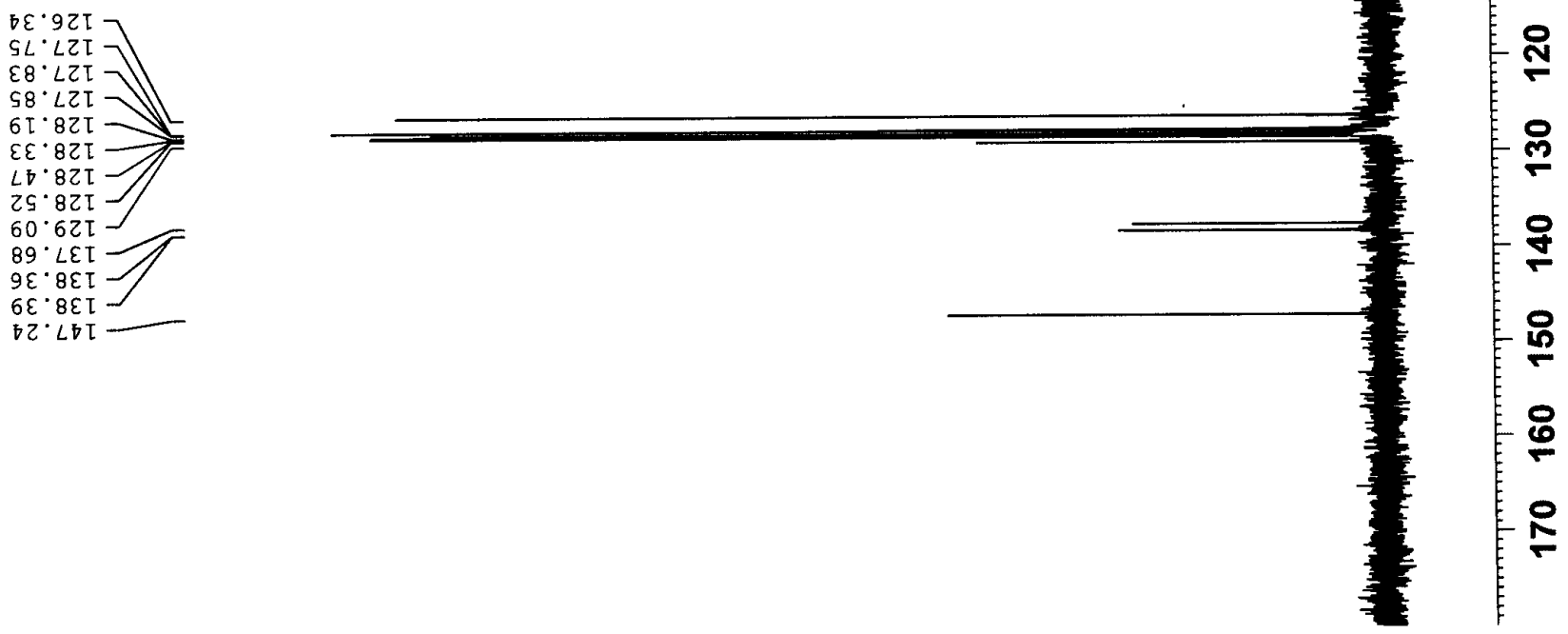

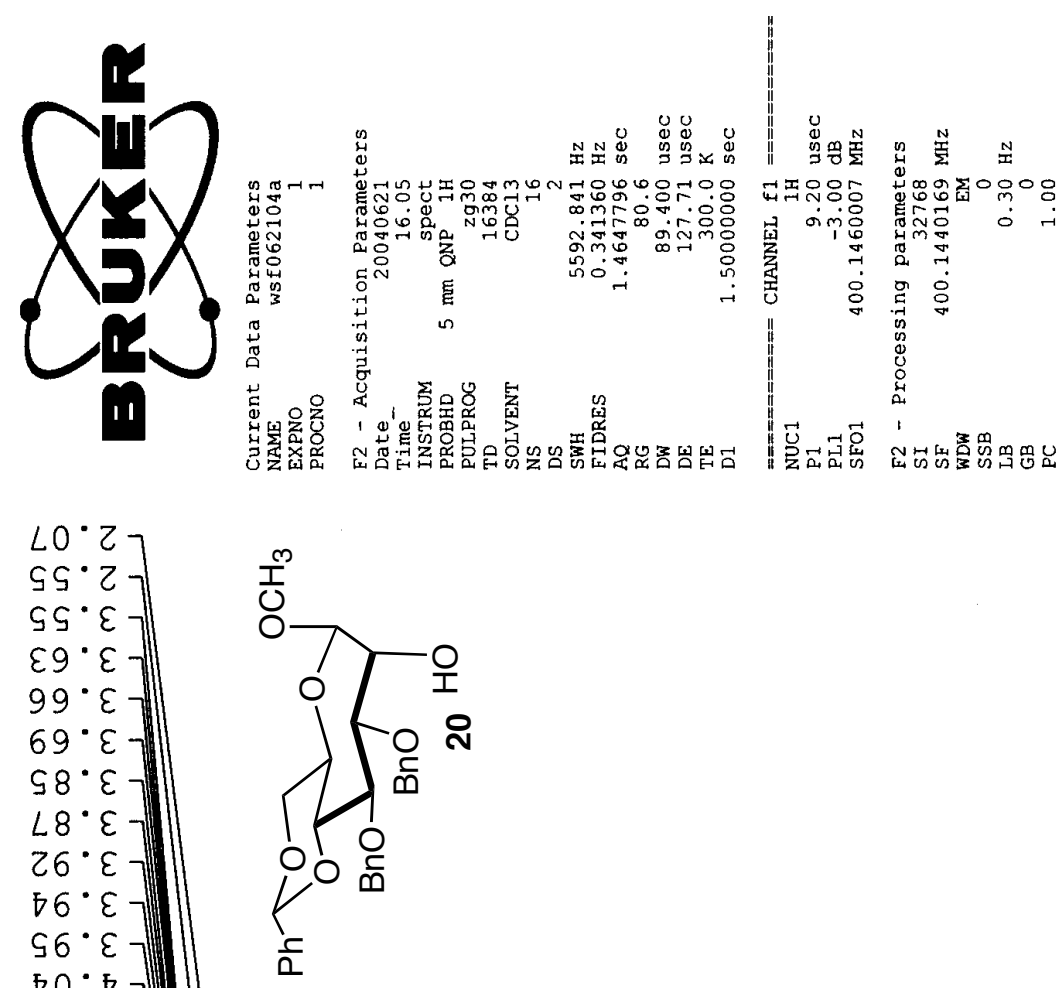

$50 \cdot \nabla]$

$\mathrm{SO}^{\circ}$

$2 \varepsilon \cdot \nabla$

$\varepsilon \varepsilon \cdot \nabla$

$\nabla \varepsilon \cdot$

$\varepsilon 9^{\circ}$

$99^{\circ}$

$69^{\circ}$

IL.

$Z L^{\circ} \cdot \bar{\sigma}$

$0 \varepsilon \cdot L]$

$\left[\varepsilon^{\circ} L\right]$

$Z \varepsilon^{\bullet} L$

$\varepsilon \varepsilon$

$\nabla \varepsilon \cdot L][$

$\nabla \varepsilon^{\circ}\llcorner]$

$9 \varepsilon^{\circ} L$

$9 \varepsilon^{\circ} L$

$L \varepsilon^{\circ} L$

$8 \varepsilon^{\circ} L$

$6 \varepsilon^{\circ} L$

$0 \sigma^{\circ} L$

$00^{\circ} L$

I ${ }^{\circ} L$

$2 \nabla \cdot L$

$\varepsilon \nabla \cdot L-$

$O S^{\circ} L-$

$0 S^{\circ} L J$

$2 S^{\circ} L-$

$2 S^{\circ} L$
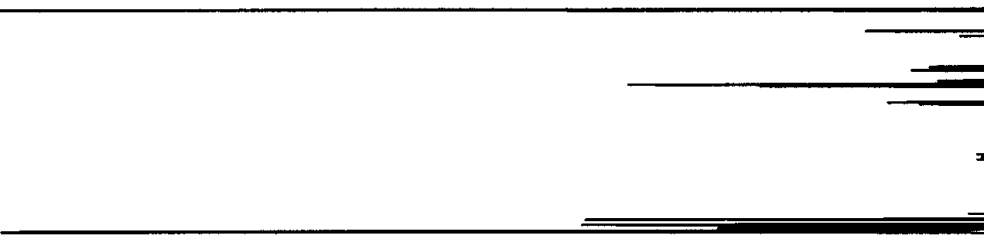

E E 틍

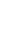

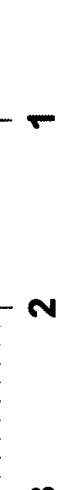

$m$

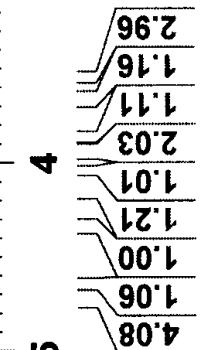

$=00 \%$

0

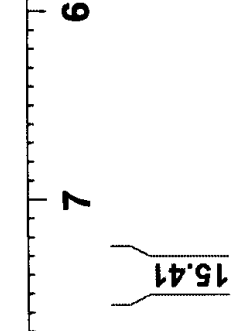

$\infty$

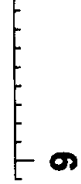



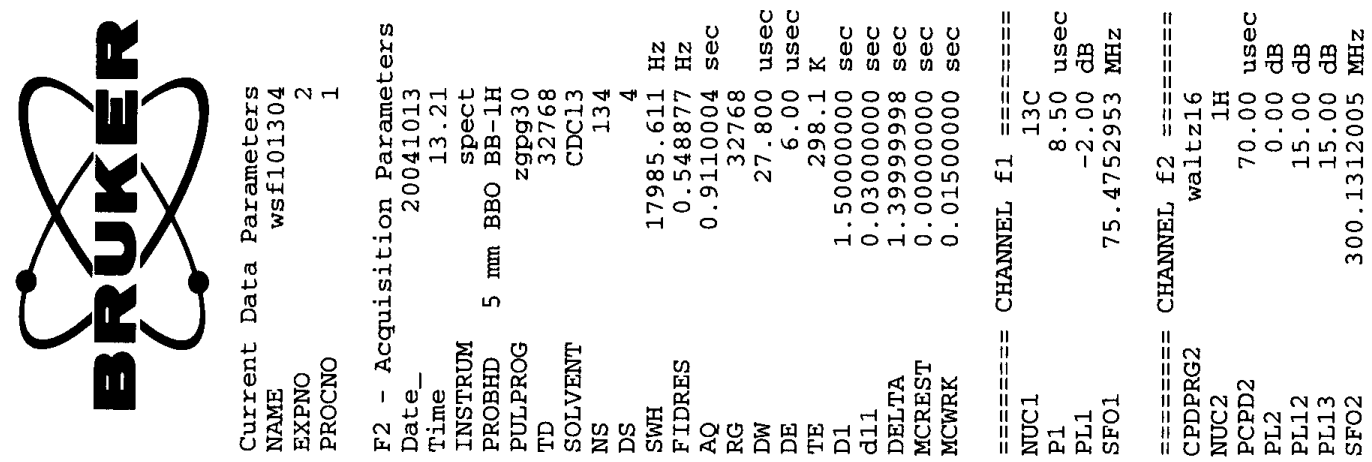

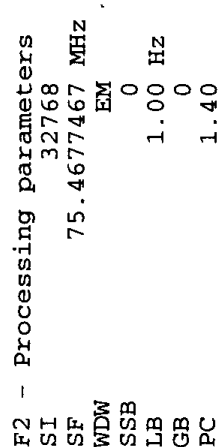
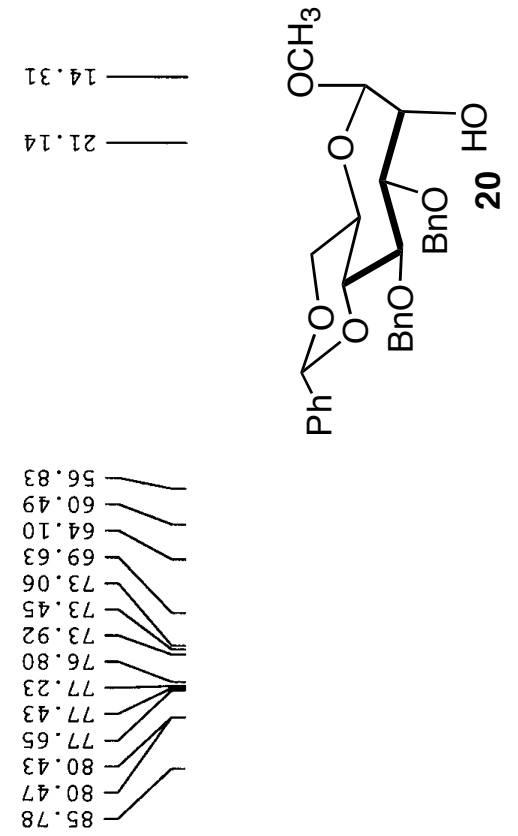

$28^{\circ} 00 \mathrm{I}$

$\varsigma^{\circ} \varepsilon 0 \mathrm{~T}$

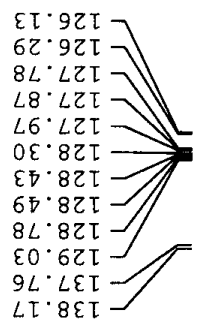

$\varepsilon \tau \cdot \tau L I-$
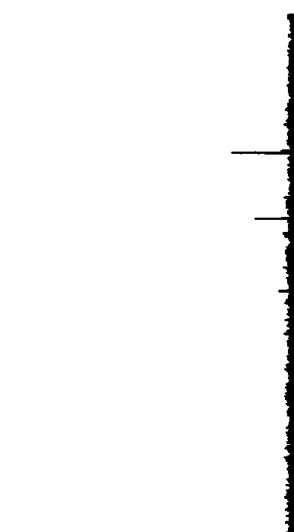

틍

오

이

웅

오

8

R

\&

8

으

$=$

옹

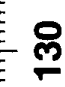

q

운

\&

옹 

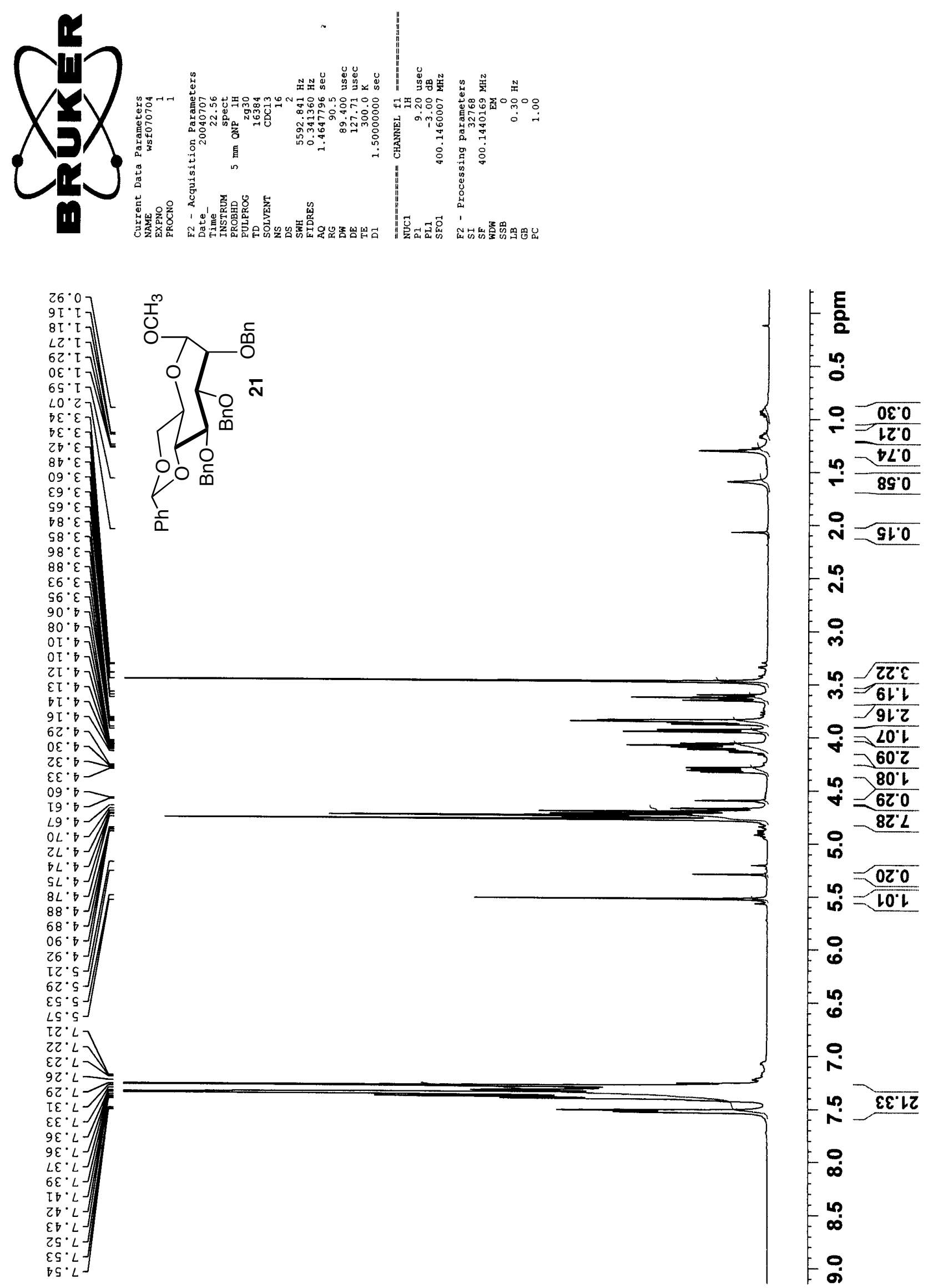

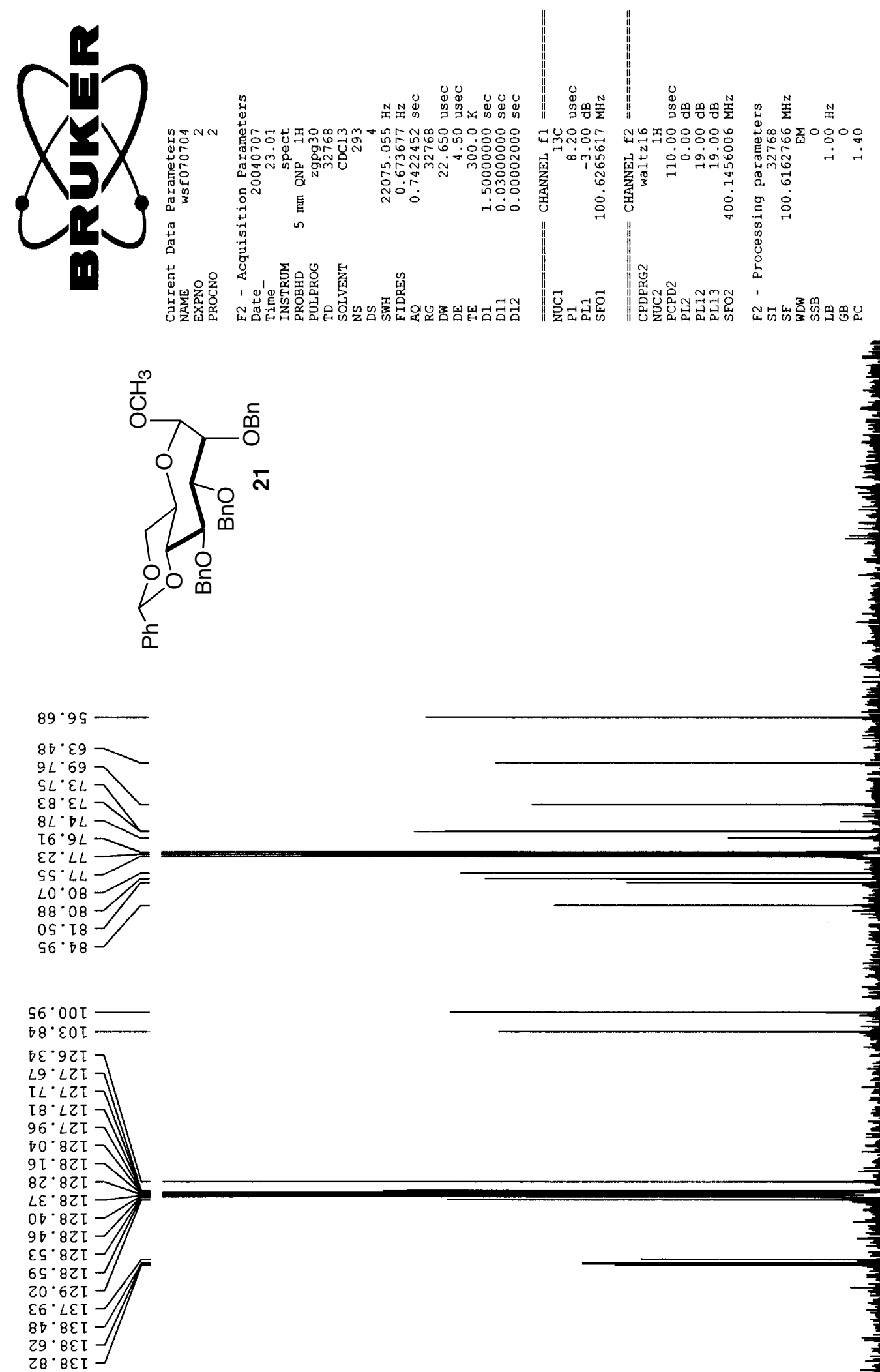

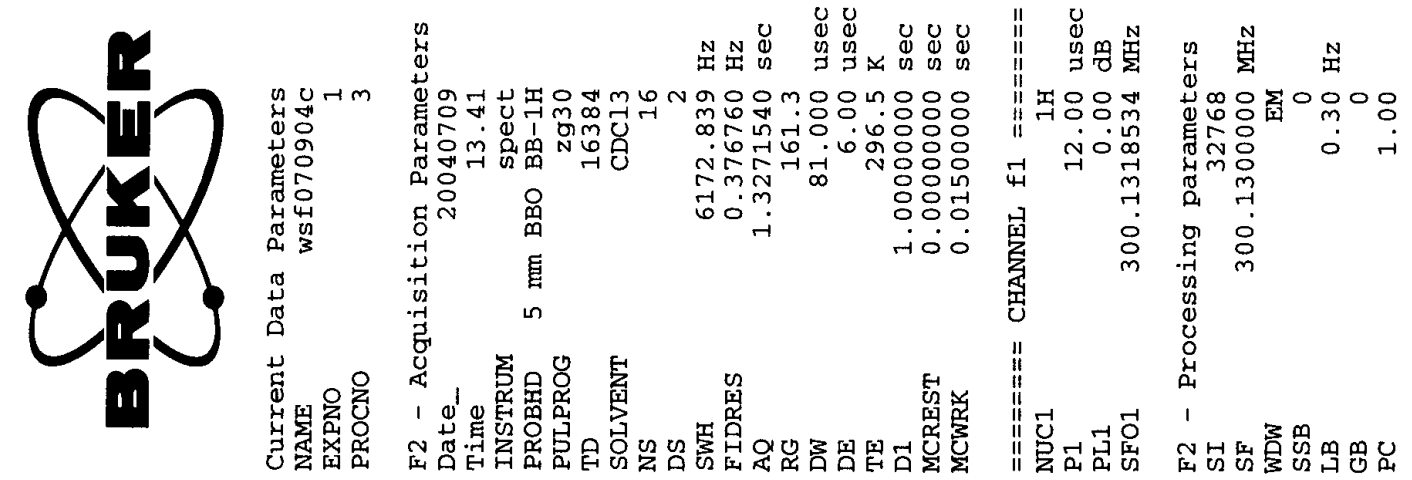

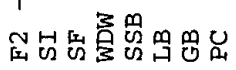
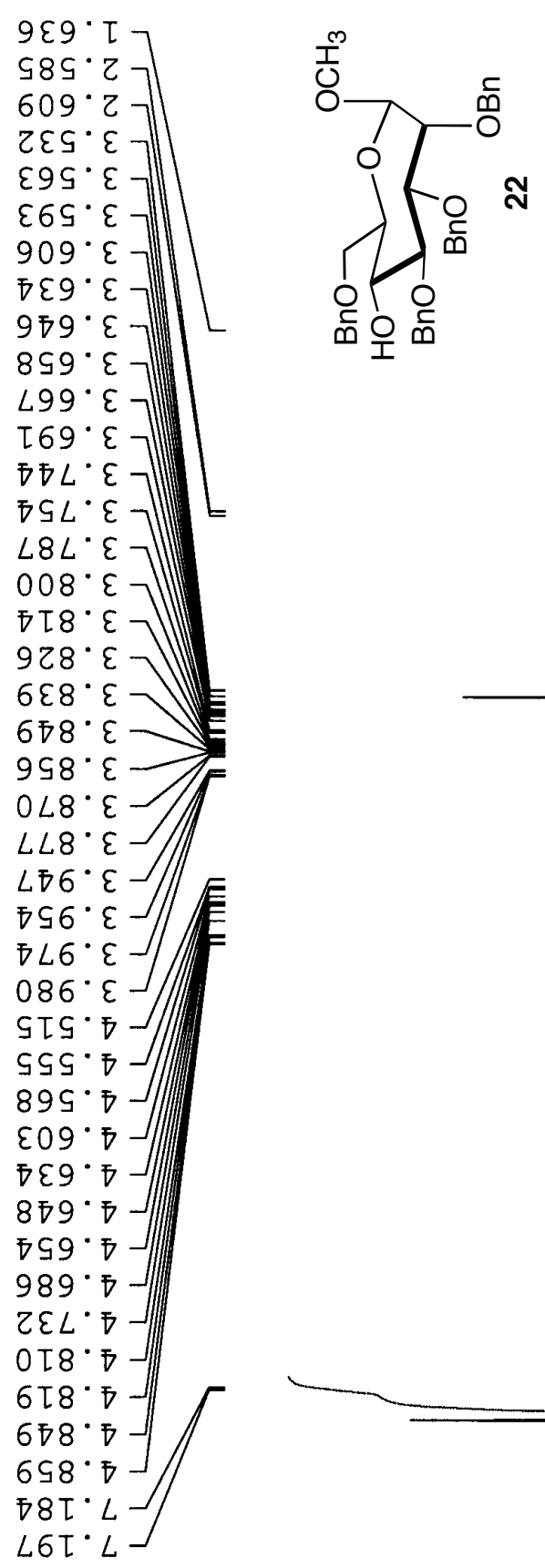


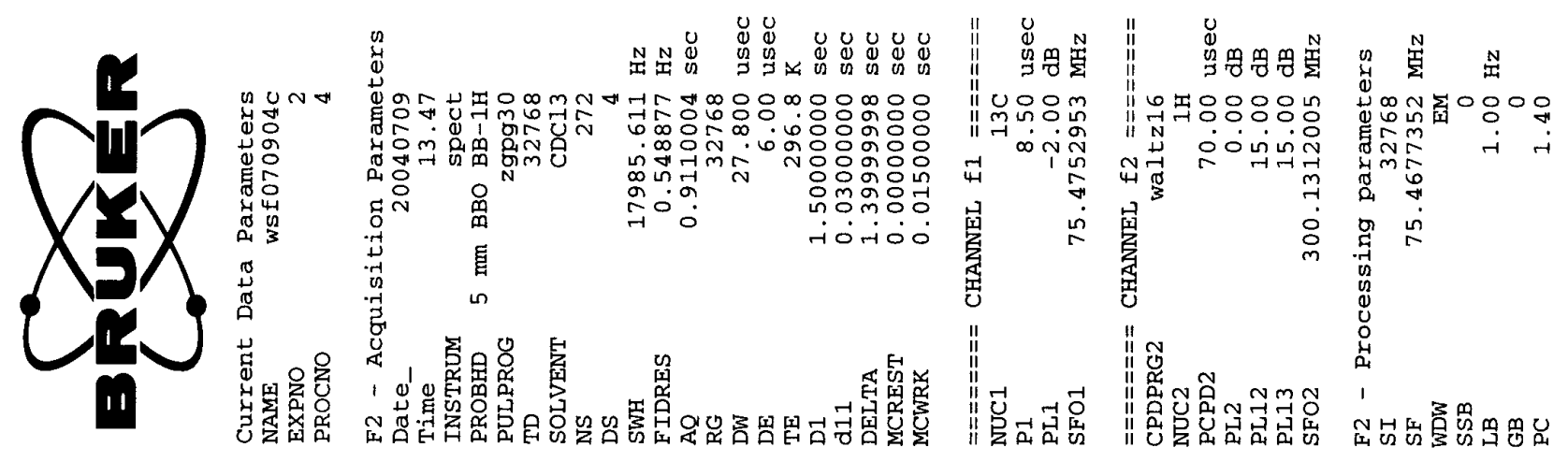

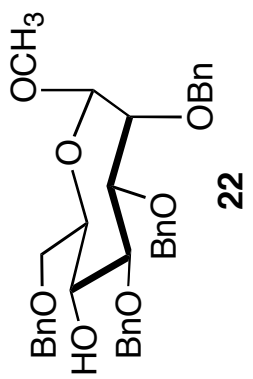
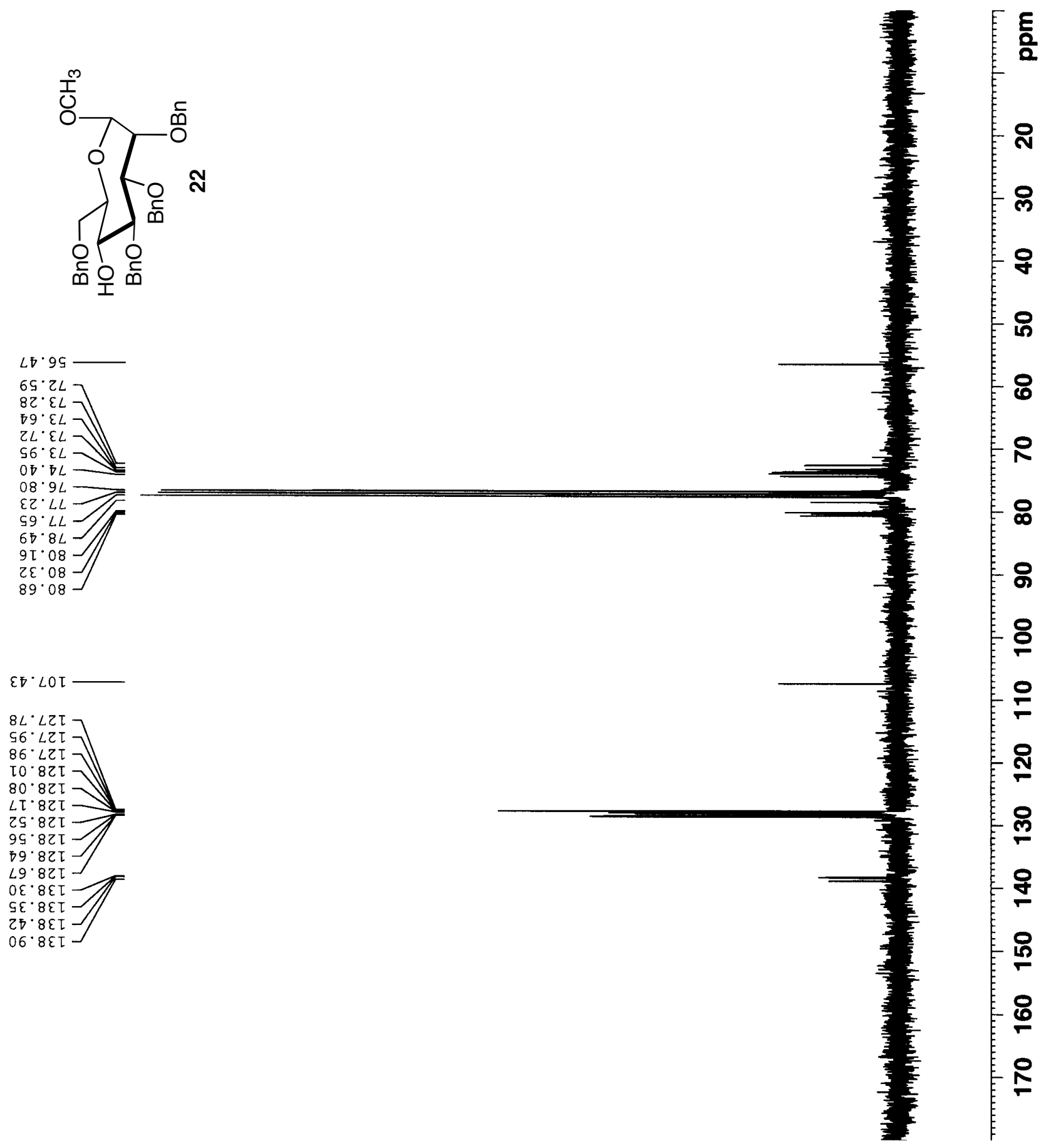

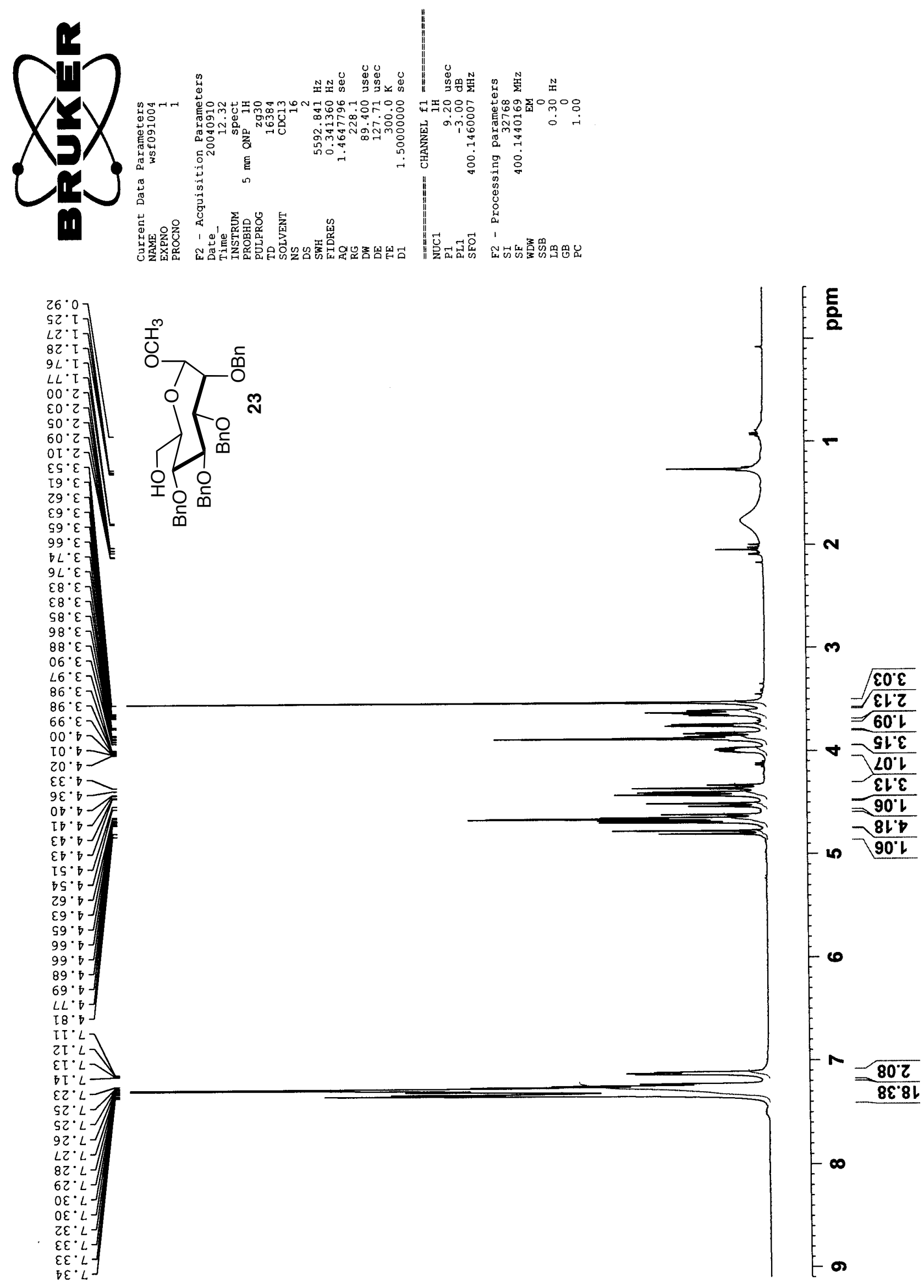


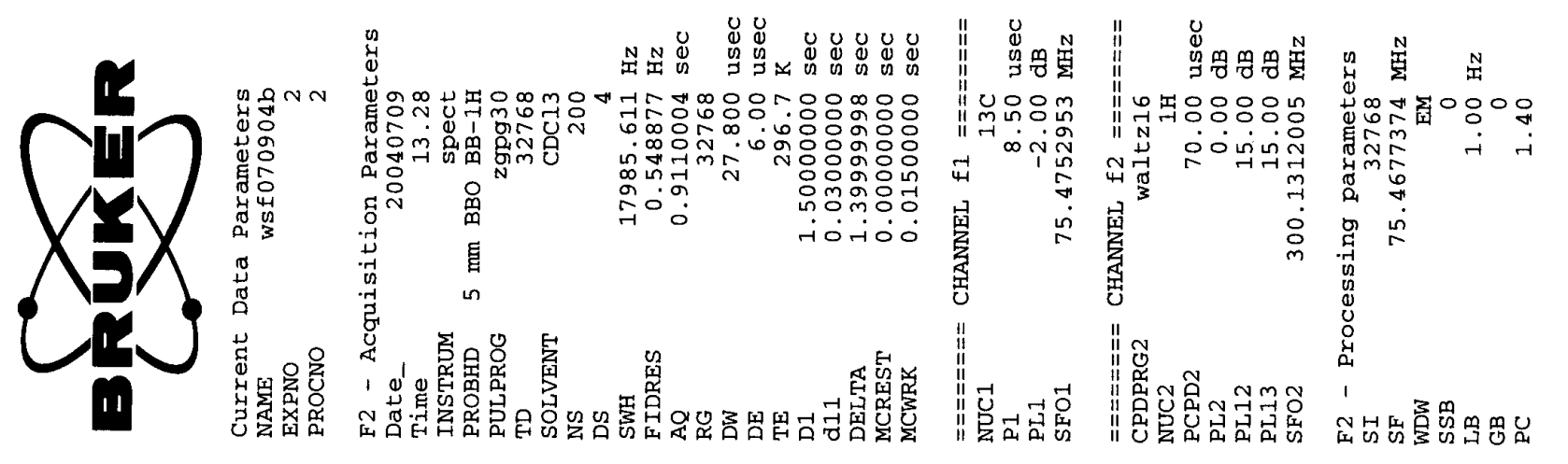

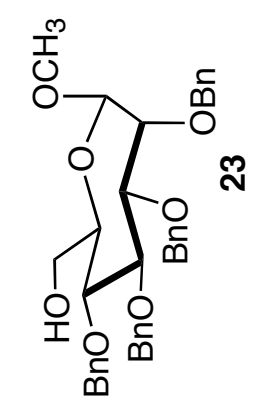
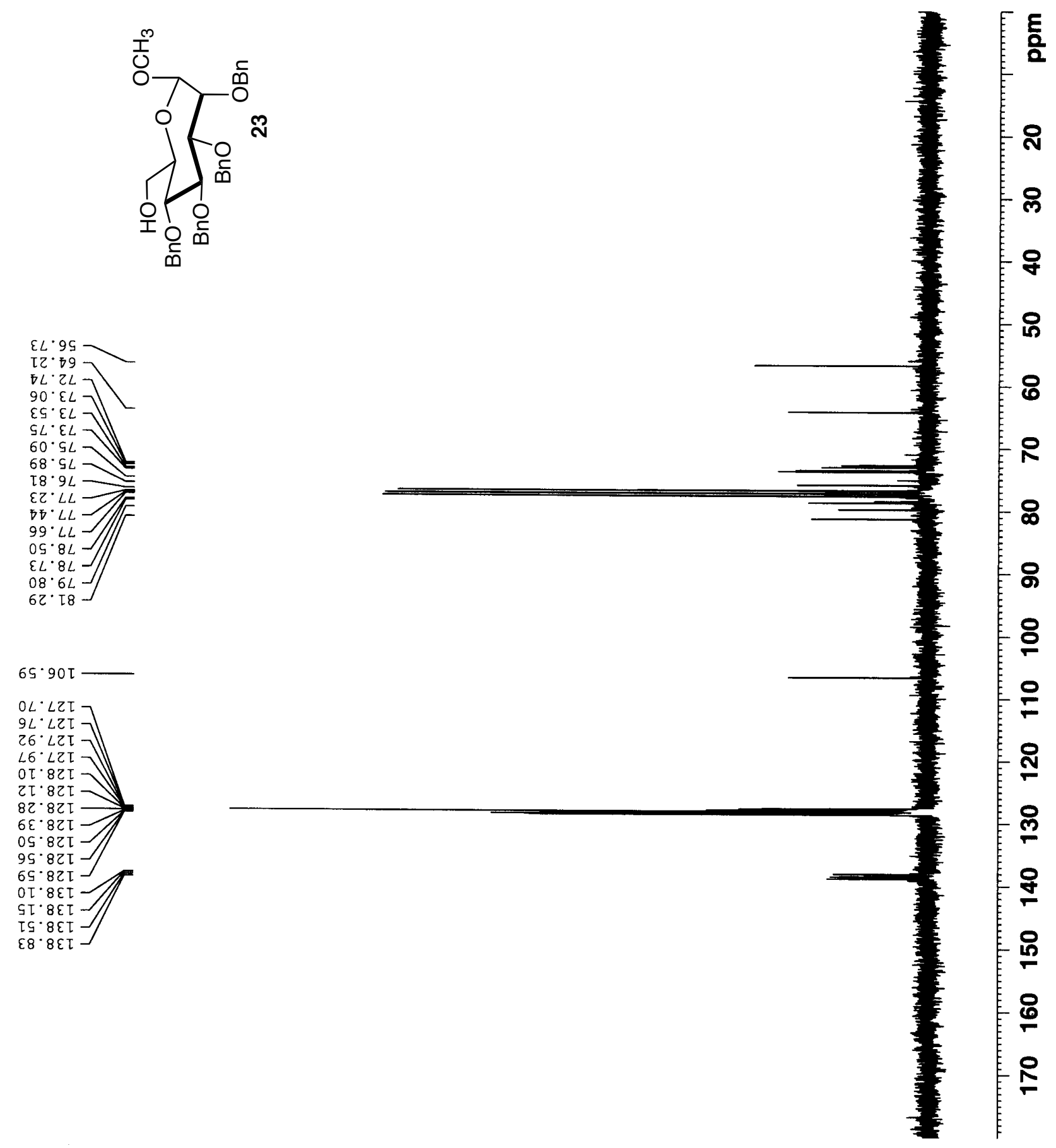


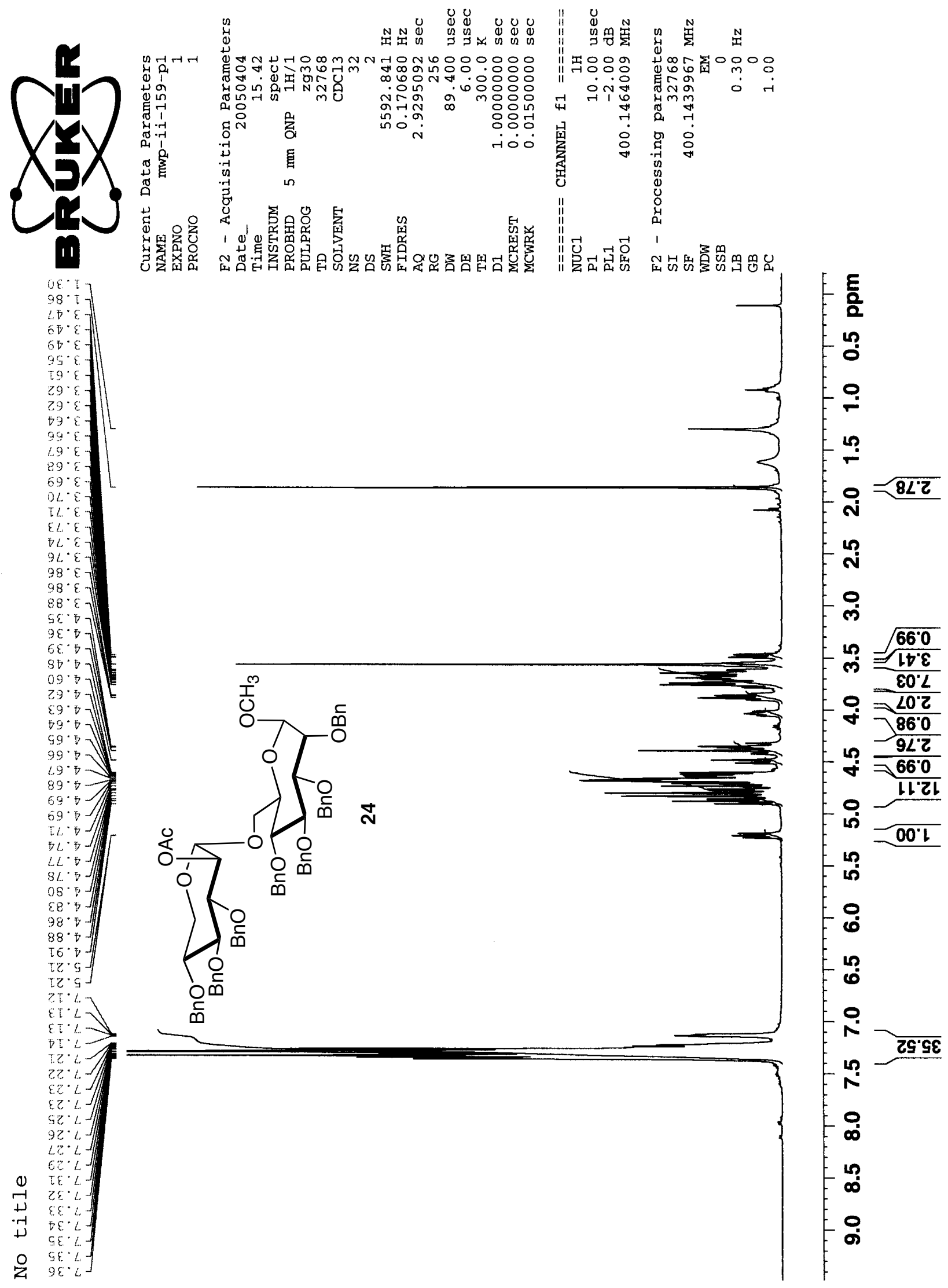




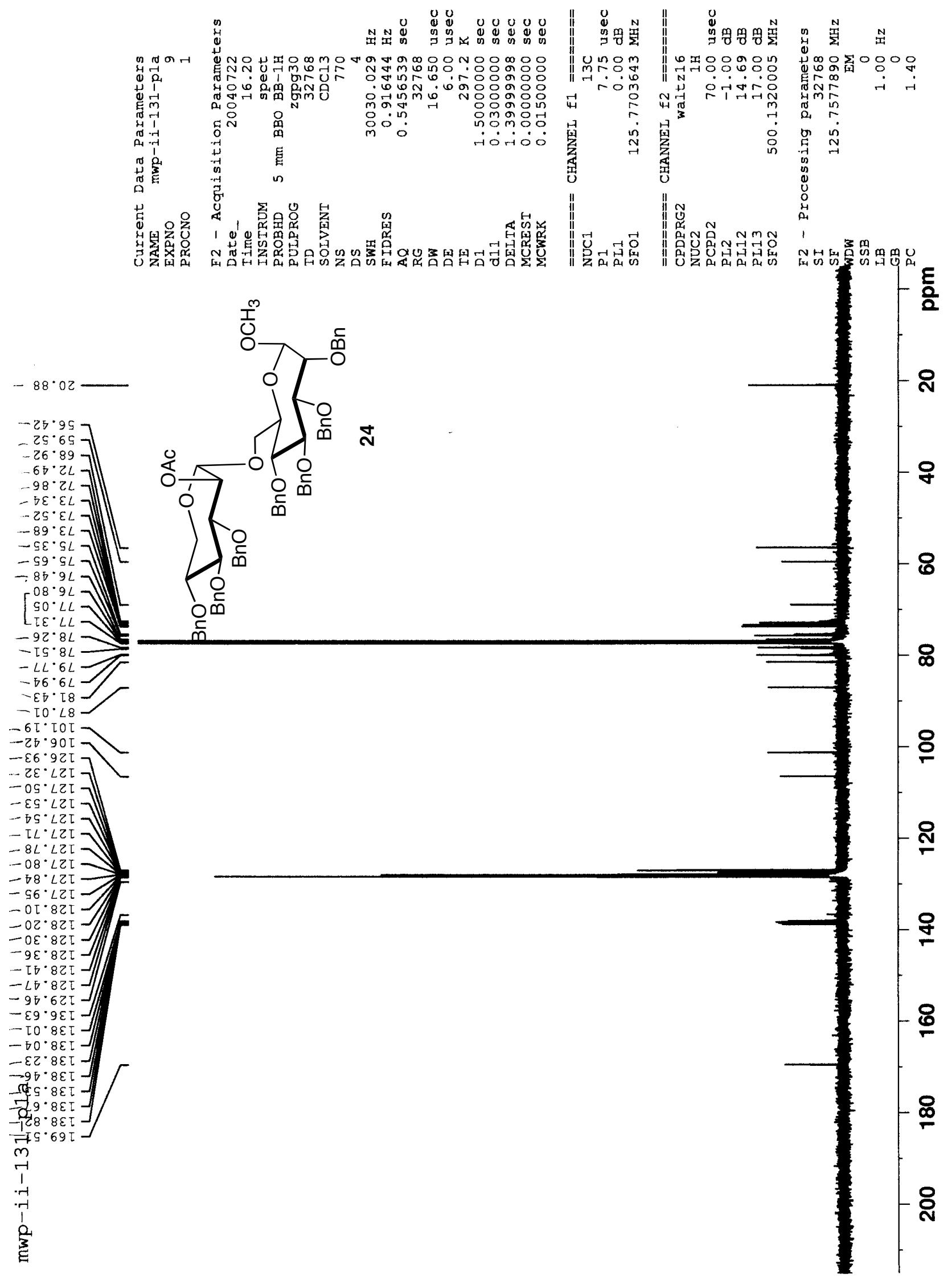



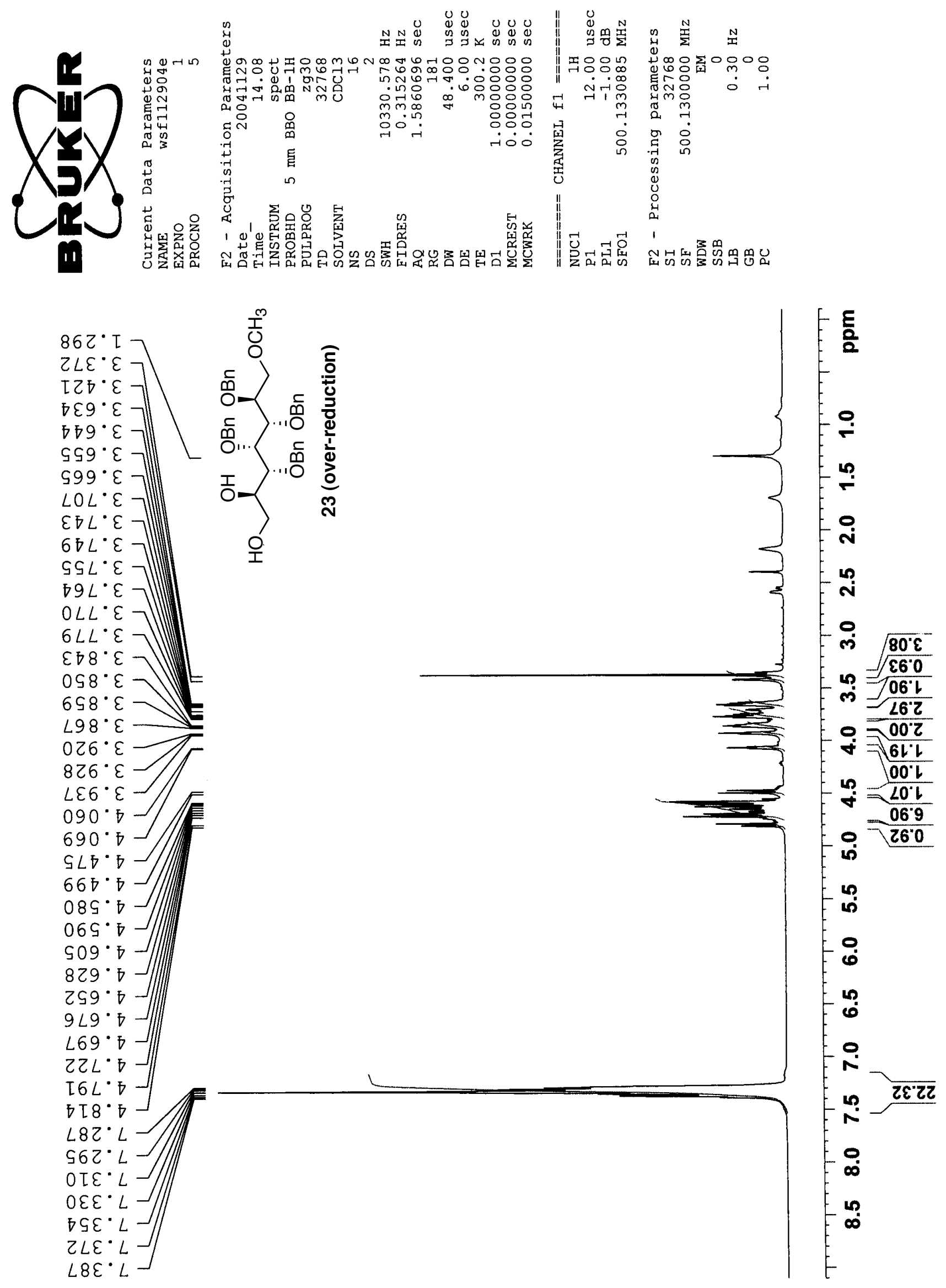

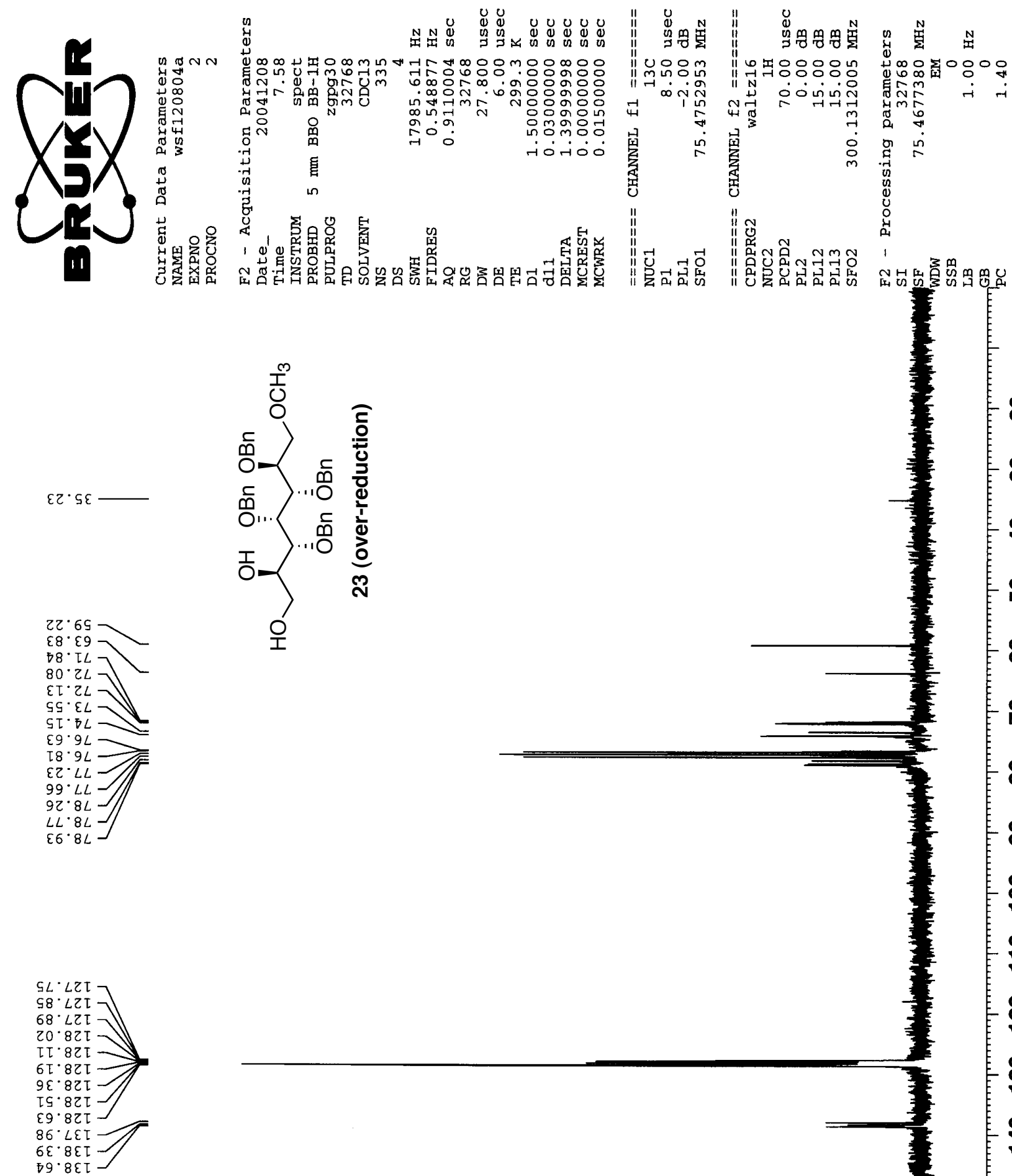